\title{
2010
}

\section{Savannah River Site}

Annual IIIness and Injury

Surveillance Report

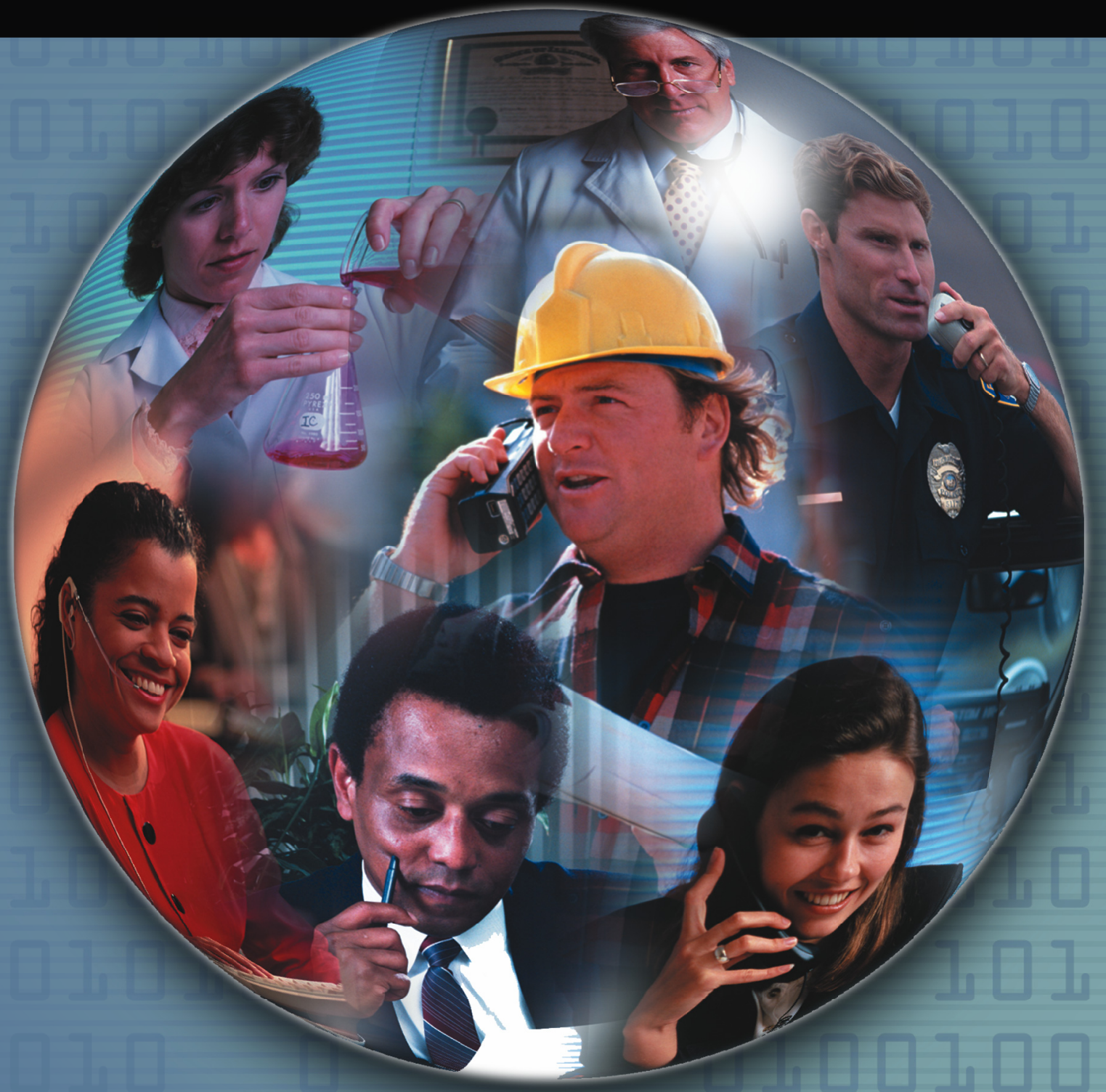




\section{Savannah River Site 2010 Illness and Injury Surveillance Report}

Questions or comments about this report or the Illness and Injury Surveillance Program (IISP) may be directed to:

E-mail:

Dr. Cliff Strader at cliff.strader@hq.doe.gov or Dr. Bonnie Richter at bonnie.richter@hq.doe.gov

or direct letters to:

Mail Stop HS-13 / GTN Building

U.S. Department of Energy

1000 Independence Avenue, S.W.

Washington, DC 20585-0270

Additional information about the Department of Energy's Office of Illness and Injury Prevention Programs, the IISP, and annual reports for DOE sites participating in this program can be found at:

http://www.hss.energy.gov/healthsafety/WSHP/epi/surv/

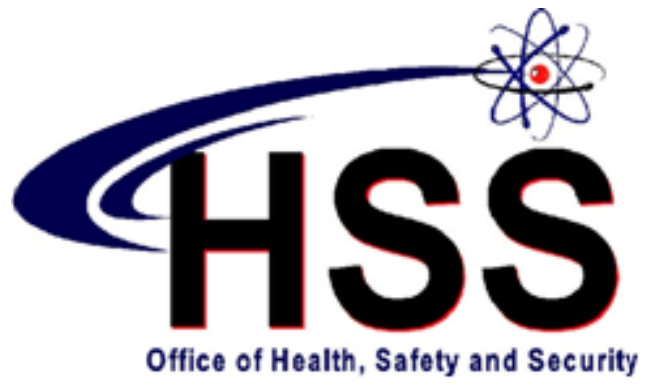

ACKNOWLEDGEMENT

LifeART images copyright 2000 Lippincott Williams \& Wilkins. All rights reserved.

This document was produced under contract number DE-AC05-06OR23100 between the

U.S. Department of Energy and Oak Ridge Associated Universities. 


\section{Savannah River Site \\ 2010 Illness and Injury Surveillance Report}

At A Glance

NOTE: In previous years, the number of days absent or lost and the age of the work force were reported as averages. Beginning in 2010, they are being reported as medians. A median is the value in a population that half the values exceed and half fall short of it. The median is less affected than the average when the distribution contains a few very large or very small values and provides a more representative summary measure.

The 9,621 employees at Savannah River Site (SRS) in 2010 represented a decrease of 2 percent from 2009, the first decrease in workers at SRS since 2007. Compared with the work force in 2007 (8,948 workers, the lowest number of workers at SRS over the period), workers in 2010 increased 8 percent. The median age of Savannah River Site workers in 2010, 51 years for men and 50 years for women, was the same as in 2009. The median age of SRS workers in 2006 was 48 years for both men and women. Women have accounted for approximately 25 percent of the work force since 2006 .

Savannah River Site reported 3,152 absences in 2010, a 17 percent decrease from the 3,800 absences reported in 2009. This decrease is not related to gender, age, or occupational group. This is the first year that absences have decreased at SRS since 2006, when 3,022 absences were reported.

In 2010, the absence rate was 48 per 100 women and 28 per 100 men. The higher absence rate among women is consistent with SRS absence rates since 2006. In general, women reflect higher absence rates than do men across the sites participating in the Illness and Injury Surveillance Program (IISP). The median length of absence among SRS workers was 7 days for both women and men in 2010, similar to all years since 2006.

Women in the work force lost 15,517 calendar days due to illness and injury in 2010. Respiratory conditions (19 percent), musculoskeletal conditions (17 percent), and unspecified symptoms (20 percent) accounted for 56 percent of all reported diagnoses. Respiratory symptoms, fever, and abdominal pain were responsible for 56 percent of the unspecified symptoms. 
Men lost 29,819 calendar days due to illness and injury. Over half of their reported diagnoses (55 percent) involved respiratory conditions (18 percent), musculoskeletal conditions (18 percent), and unspecified symptoms (19 percent). More than half of the unspecified symptoms (53 percent) were respiratory symptoms, abdominal pain, and fever.

Respiratory and musculoskeletal disorders and unspecified symptoms were the top 3 diagnosis categories reported by men and women at SRS from 2006 to 2010. Diagnosis rates for disorders of the respiratory and musculoskeletal systems were among the highest observed for all DOE IISP sites from 2000 to 2009.

Line Operators had the highest absence rate among both women and men. Crafts workers had the lowest absence rate among women and Service workers had the lowest rate among men. The high rate among Line Operators was typical of the pattern noted among all IISP sites as a group from 2000 through 2009. Women in the Security and Fire occupational category reported no absences in 2010; the Security workers in this group have not participated in IISP for security reasons.

In 2010, SRS workers reported 12 definite and 54 possible sentinel health event (SHEO) diagnoses. Carpal tunnel syndrome (CTS) accounted for 32 of these diagnoses (all categorized as possible SHEO diagnoses), reported by 26 workers. CTS resulted in a total of 475 days absent. All but 1 worker reporting CTS were aged 40 and above. CTS was most frequently reported by Line Operators and Professional and Technical Support workers. Three diagnoses of chronic beryllium disease were reported, all considered definite SHEOs. No particular diagnostic category predominated among the remaining SHEO diagnoses.

In 2010, SRS reported 23 OSHA events, less than half the number of events reported in 2009, mainly due to a decrease in OSHA events among Line Operators. The number of lost or restricted workdays reported in 2010 decreased to 694 days, one-third fewer days than reported in 2009. 
Nine of the 22 workers (40.9 percent) reporting an OSHA event were Crafts workers, an occupational group comprising only 12 percent of the work force. All but 1 of the OSHA events were classified as accidents. Overexertion and strenuous movements, accidents caused by a cutting/piercing instrument/object, and natural/environmental factors accounted for over half of these accidents. 
The Savannah River Site

Work Force - 2010

The Work Force by Gender and Age ............ 1

The Work Force by Gender and Job

Category .. 1

\section{Number and Length of Absences}

Absence Rate by Gender and Age 2

Number of Days Absent by

Gender and Age

Absence Rate by Job Category

and Gender .. 3

Median Duration of Absence by

Job Category and Gender. .. 3

\section{Diagnostic Categories}

Number of Diagnoses and Lost Calendar Days by Diagnostic Category

(Categorized by ICD-9-CM) and Gender . .4

Common Diagnoses Among Female

Workers in 2010 .... . .5

Common Diagnoses Among Male

Workers in 2010 . 6

Number of Most Frequently Reported Diagnoses by Job Category and Gender 7

\section{Rates of Disease Occurrence}

Rates for All Illnesses and Injuries Combined by Job Category, Gender, and Age

Rates for Selected Diagnostic Categories by Job Category, Gender, and Age 8

\section{Time Trends}

Age-Adjusted Rates for All Diagnoses Combined Among Women and Men from 2006 to 2010
Age-Adjusted Rates for Selected Diagnostic Categories Among Women and Men from 2006 to 2010

Age-Adjusted Rates for All Diagnoses

Combined Among Women and Men by Job

Category from 2006 to $2010 \ldots \ldots \ldots \ldots \ldots \ldots \ldots \ldots . \ldots 12$

\section{Sentinel Health Events for Occupations (SHEOs)}

Characteristics of SHEOs by Gender 13

SHEO Diagnoses by Gender 13

\section{Occupational Safety and Health Administration (OSHA)-Recordable Events}

OSHA-Recordable Events by Gender and Age. 14

OSHA-Recordable Events by Job

Category and Gender 14

\section{Diagnostic and Accident Categories for OSHA-Recordable Events}

OSHA-Recordable Diagnoses by

Diagnostic Category and Gender

OSHA-Recordable Accidents by Type

and Gender

\section{Rates of OSHA-Recordable Events}

OSHA-Recordable Rates by Age and Job

Categories Among Women, All Diagnoses

Combined

OSHA-Recordable Rates by Age and Job

Categories Among Men, All Diagnoses

Combined

Time Trends for OSHA-Recordable Events

Age-Adjusted Rates for All OSHA-Recordable Diagnoses Combined Among Women and Men by Job Category from 2006 to 2010 .

\section{Appendices}

Appendices A-W .20 
The Savannah River Site Work Force - 2010

Figure 1. The Work Force by Gender and Age

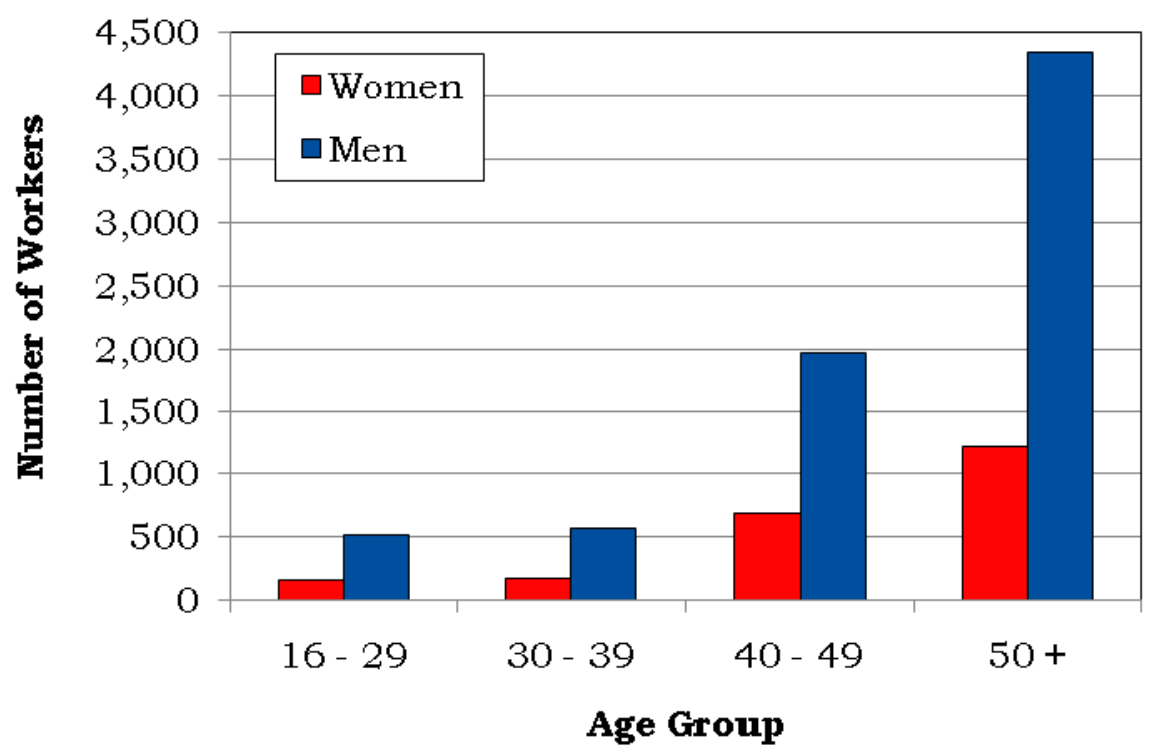

Figure 2. The Work Force by Gender and Job Category

\begin{tabular}{|l|c|c|}
\hline \multicolumn{1}{|c|}{ Job Category } & Women & M en \\
\hline Professional & 638 & 2,073 \\
& $28 \%$ & $28 \%$ \\
\hline \multirow{2}{*}{ Administrative Support } & 542 & 327 \\
& $24 \%$ & $4 \%$ \\
\hline \multirow{2}{*}{ Technical Support } & 633 & 2,245 \\
& $28 \%$ & $30 \%$ \\
\hline \multirow{2}{*}{ Service } & 18 & 292 \\
& $1 \%$ & $4 \%$ \\
\hline \multirow{2}{*}{ Security and Fire } & 3 & 37 \\
& $<1 \%$ & $1 \%$ \\
\hline \multirow{2}{*}{ Crafts } & 133 & 1,043 \\
& $6 \%$ & $14 \%$ \\
\hline \multirow{2}{*}{ Line Operators } & 261 & 1,376 \\
& $12 \%$ & $19 \%$ \\
\hline Total & 2,228 & 7,393 \\
& $100 \%$ & $100 \%$ \\
\hline
\end{tabular}




\section{Number and Length of Absences}

Figure 3. Absence Rate by Gender and Age

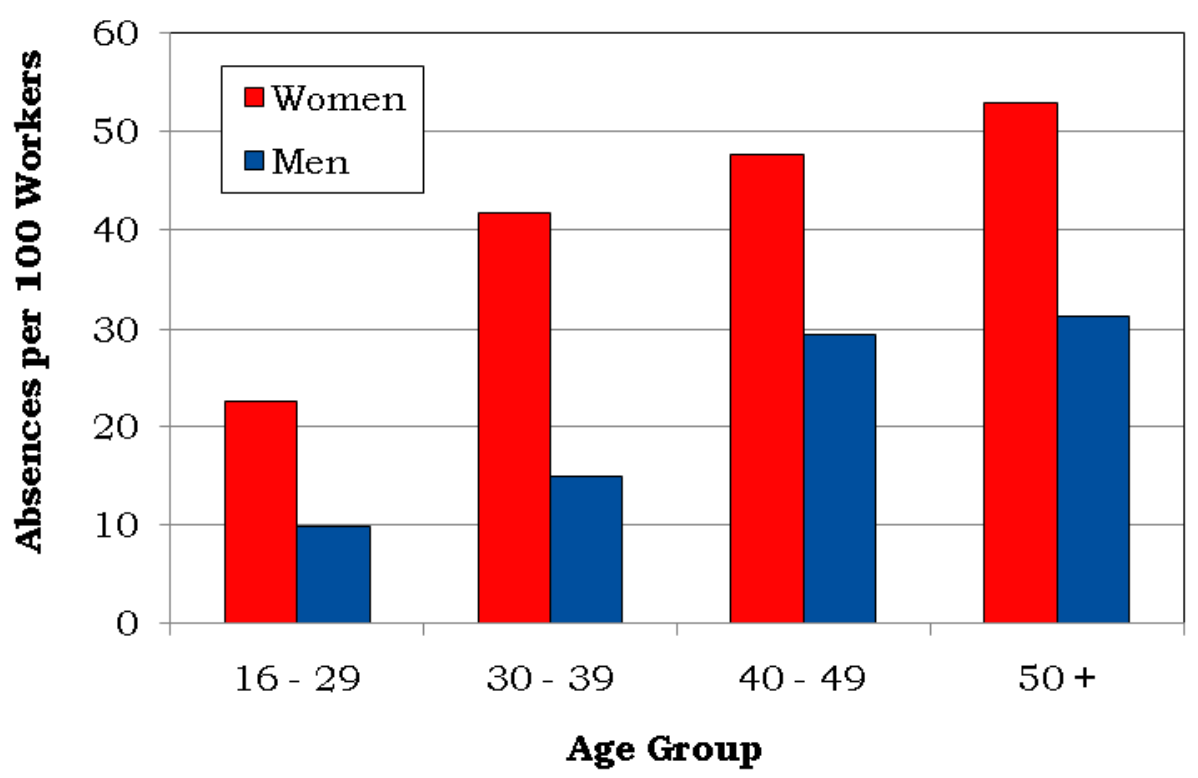

Figure 4. Number of Days Absent by Gender and Age

\begin{tabular}{|c|c|c|c|c|}
\hline \multirow{2}{*}{ Gender } & \multirow{2}{*}{ Age } & \multirow{2}{*}{$\begin{array}{l}\text { Number of } \\
\text { Absences }\end{array}$} & \multicolumn{2}{|c|}{ Number of Days Absent } \\
\hline & & & Total & Median \\
\hline \multirow{5}{*}{ Women* } & $16-29$ & 36 & 302 & 5 \\
\hline & $30-39$ & 73 & 890 & 7 \\
\hline & $40-49$ & 325 & 4,518 & 7 \\
\hline & $50+$ & 641 & 9,807 & 7 \\
\hline & Total & 1,075 & 15,517 & 7 \\
\hline \multirow{5}{*}{ Men } & $16-29$ & 51 & 522 & 7 \\
\hline & $30-39$ & 84 & 962 & 7 \\
\hline & $40-49$ & 581 & 7,595 & 7 \\
\hline & $50+$ & 1,361 & 20,740 & 7 \\
\hline & Total & 2,077 & 29,819 & 7 \\
\hline
\end{tabular}

*Normal pregnancies were excluded from absences for women. 
Figure 5. Absence Rate by Job Category and Gender

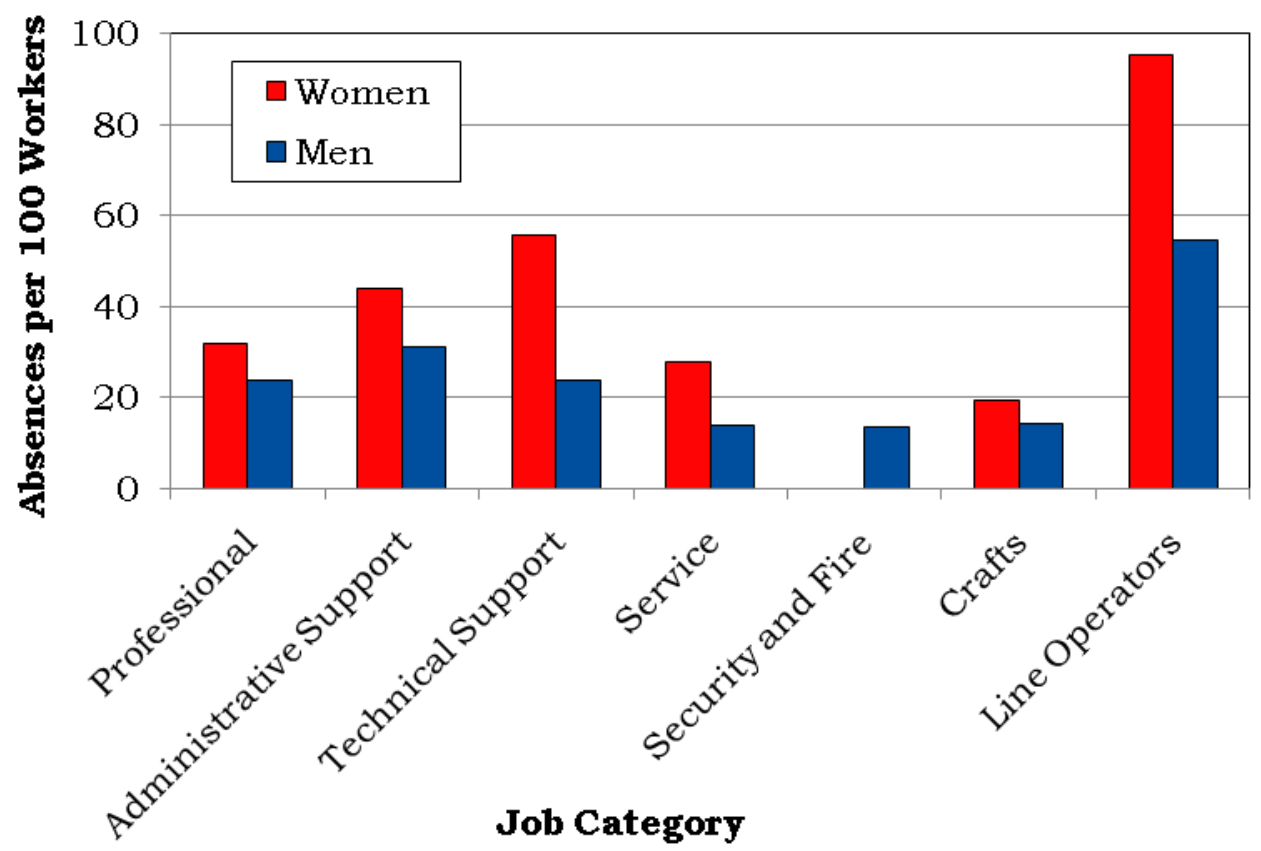

Figure 6. Median Duration of Absence by Job Category and Gender

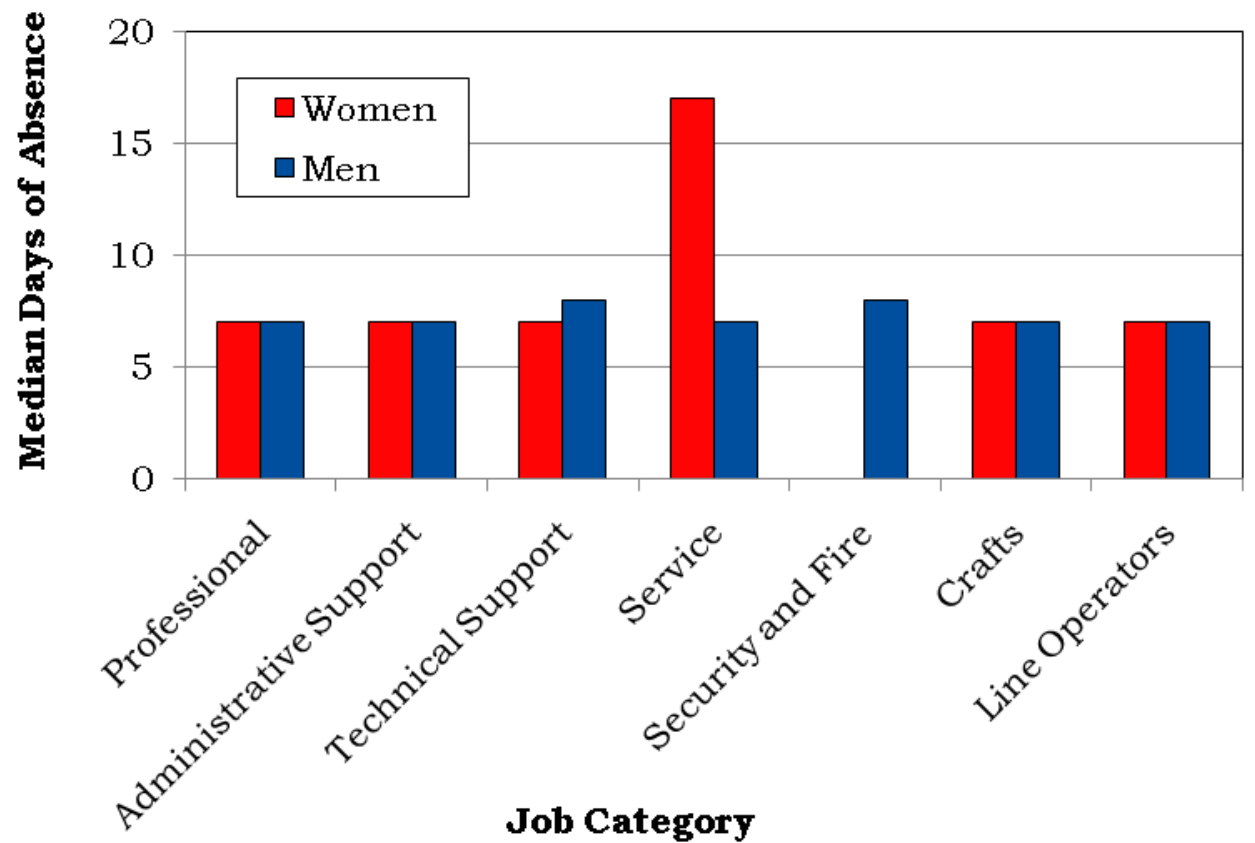




\section{Diagnostic Categories}

Figure 7. Number of Diagnoses and Lost Calendar Days by Diagnostic Category (Categorized by ICD-9-CM) and Gender

\begin{tabular}{|c|c|c|c|c|}
\hline \multirow[b]{2}{*}{ Diagnostic Category } & \multicolumn{2}{|c|}{ Women } & \multicolumn{2}{|c|}{ Men } \\
\hline & $\begin{array}{l}\text { Number of } \\
\text { Diagnoses }\end{array}$ & $\begin{array}{c}\text { Number } \\
\text { of Lost } \\
\text { Calendar } \\
\text { Days }\end{array}$ & $\begin{array}{l}\text { Number of } \\
\text { Diagnoses }\end{array}$ & $\begin{array}{c}\text { Number } \\
\text { of Lost } \\
\text { Calendar } \\
\text { Days }\end{array}$ \\
\hline Benign Growths & 42 & 970 & 29 & 457 \\
\hline Blood & 23 & 243 & 29 & 484 \\
\hline Cancer & 66 & 1,216 & 76 & 1,703 \\
\hline Digestive & 133 & 1,570 & 260 & 3,303 \\
\hline Endocrine / Me tabolic & 47 & 689 & 132 & 1,876 \\
\hline Existing Birth Condition & 4 & 207 & 7 & 170 \\
\hline Genitourinary & 148 & 1,922 & 143 & 1,421 \\
\hline Heart/Circulatory & 77 & 1,366 & 282 & 4,090 \\
\hline Infections/Parasites & 69 & 796 & 140 & 1,599 \\
\hline Injury & 145 & 2,620 & 317 & 5,696 \\
\hline Miscarriage & 0 & 0 & NA & NA \\
\hline Musculoskeletal & 374 & 5,193 & 712 & 8,734 \\
\hline Nervous System & 136 & 1,511 & 202 & 2,122 \\
\hline Psychological & 30 & 487 & 87 & 1,373 \\
\hline Respiratory & 400 & 2,980 & 700 & 5,669 \\
\hline Skin & 29 & 417 & 54 & 746 \\
\hline Unspecified Symptoms & 439 & 3,497 & 762 & 5,561 \\
\hline
\end{tabular}

Note: Lost calendar days for each absence are counted more than once when multiple diagnoses occur in different diagnostic categories for the same absence. 


\section{Figure 8. Common Diagnoses Among Female Workers in 2010}

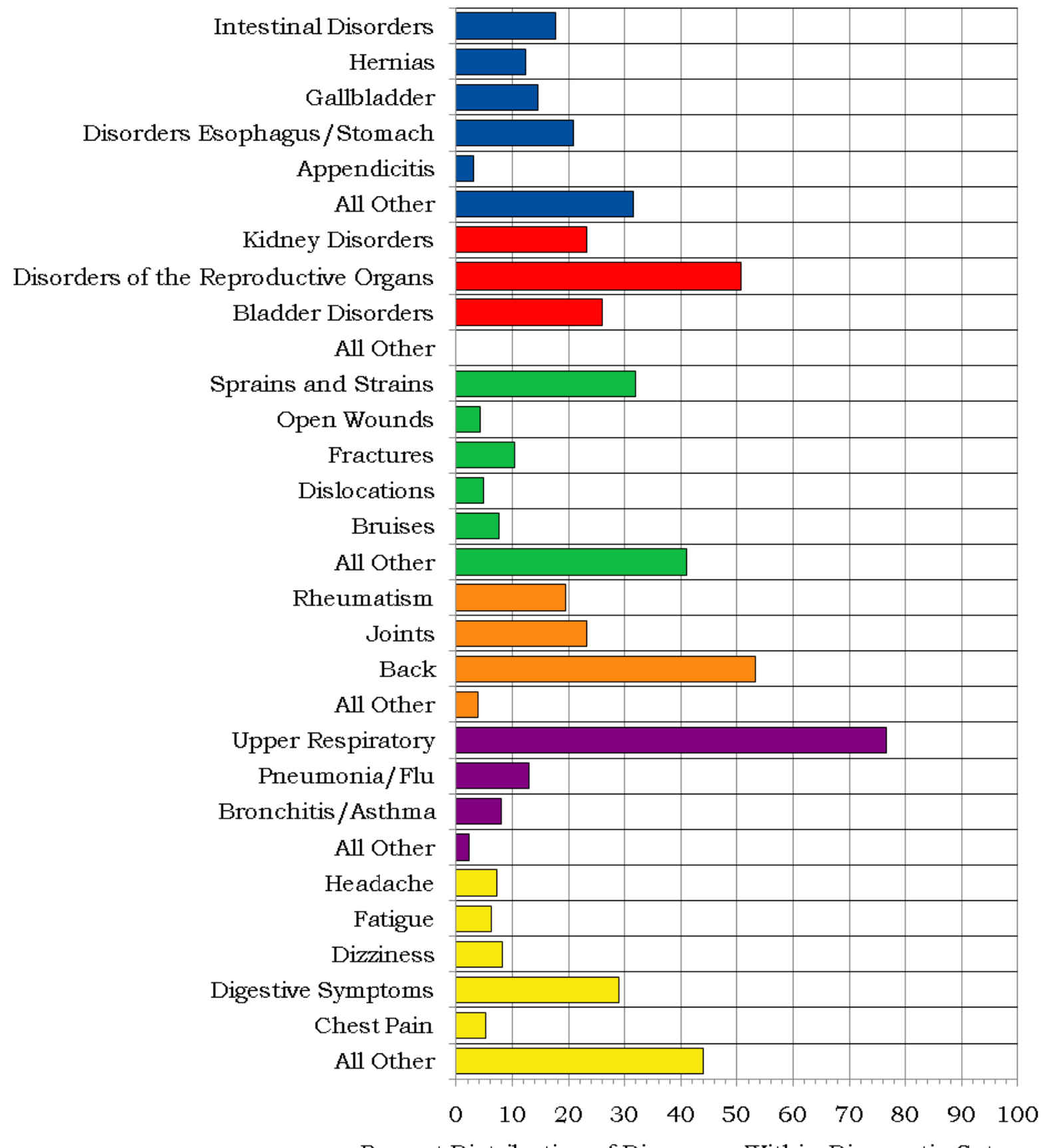

Percent Distribution of Diagnoses Within Diagnostic Category

Digestive, 130 Diagnoses

Genitourinary, 146 Diagnoses

Injury, 144 Diagnoses
Musculoskeletal, 360 Diagnoses

Respiratory, 399 Diagnoses

Unspecified Symptoms, 427 Diagnoses 


\section{Figure 9. Common Diagnoses Among Male Workers in 2010}

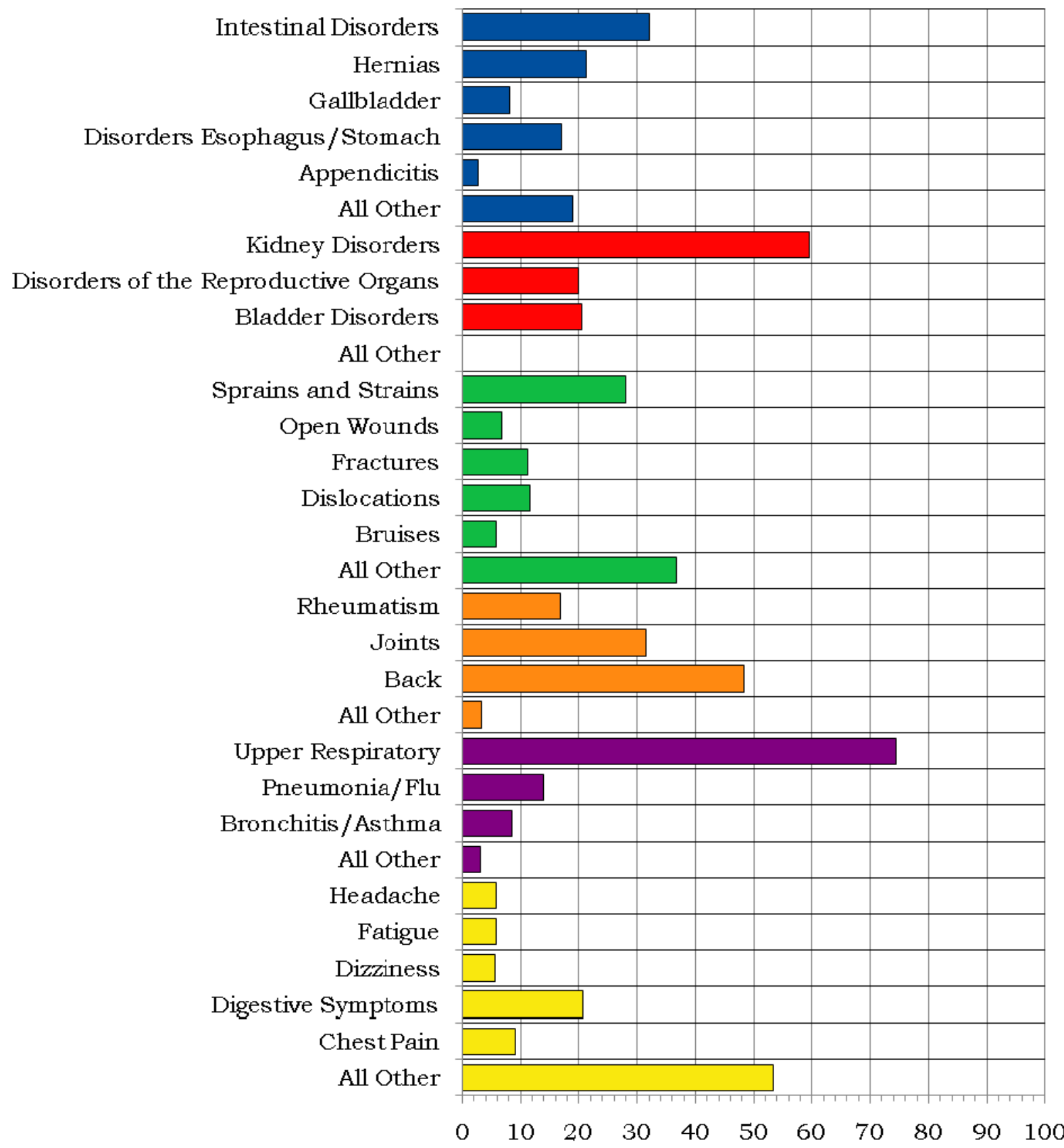

Percent Distribution of Diagnoses Within Diagnostic Category

Digestive, 259 Diagnoses

Genitourinary, 141 Diagnoses

Injury, 311 Diagnoses
Musculoskeletal, 695 Diagnoses

Respiratory, 700 Diagnoses

Unspecified Symptoms, 752 Diagnoses 


\section{Figure 10. Number of Most Frequently Reported Diagnoses by Job Category and Gender}

\begin{tabular}{|c|c|c|c|c|}
\hline Job Category & \multicolumn{2}{|l|}{ Men } & \multicolumn{2}{|l|}{ Women } \\
\hline \multirow{3}{*}{ Professional } & Respiratory & 167 & Respiratory & 82 \\
\hline & Unspecified Symptoms & 155 & Unspecified Symptoms & 70 \\
\hline & M usculoskeletal & 131 & M usculoskeletal & 59 \\
\hline \multirow{3}{*}{ Administrative Support } & M usculoskeletal & 48 & Unspecified Symptoms & 95 \\
\hline & Unspecified Symptoms & 39 & Respiratory & 83 \\
\hline & Respiratory & 24 & M usculoskeletal & 62 \\
\hline \multirow{3}{*}{ Technical Support } & Unspecified Symptoms & 202 & M usculoskeletal & 150 \\
\hline & Respiratory & 178 & Unspecified Symptoms & 134 \\
\hline & M usculoskeletal & 170 & Respiratory & 133 \\
\hline \multirow{4}{*}{ Service } & M usculoskeletal & 16 & M usculoskeletal & 3 \\
\hline & Respiratory & 11 & Unspecified Symptoms & 2 \\
\hline & Unspecified Symptoms & 10 & Digestive & 2 \\
\hline & Heart/Circulatory & 10 & Cancer & 2 \\
\hline \multirow{3}{*}{ Security and Fire } & Unspecified Symptoms & 4 & & $\mathbf{0}$ \\
\hline & Infections/Parasites & 2 & & \\
\hline & Psychological & 2 & & \\
\hline \multirow{4}{*}{ Crafts } & Unspecified Symptoms & 41 & Unspecified Symptoms & 12 \\
\hline & M usculoskeletal & 35 & M usculoskeletal & 8 \\
\hline & Respiratory & 26 & Digestive & 5 \\
\hline & Respiratory & 26 & Respiratory & 5 \\
\hline \multirow{3}{*}{ Line Operators } & M usculoskeletal & 312 & Unspecified Symptoms & 126 \\
\hline & Unspecified Symptoms & 311 & Respiratory & 97 \\
\hline & Respiratory & 293 & M usculoskeletal & 92 \\
\hline
\end{tabular}




\section{Rates of Disease Occurrence}

Figure 11. Rates for All Illnesses and Injuries Combined by Job Category, Gender, and Age

\begin{tabular}{|c|c|c|c|c|}
\hline \multirow{2}{*}{$\begin{array}{c}\text { All Illnesses \& } \\
\text { Injuries Combined }\end{array}$} & \multicolumn{4}{|c|}{ Rate per 1,000} \\
\hline & Job Category & Age & Men & Women \\
\hline & \multirow{2}{*}{ Professional } & $<50$ & 401 & 586 \\
\hline & & $50+$ & 427 & 620 \\
\hline & \multirow{2}{*}{ Administrative Support } & $<50$ & 662 & 615 \\
\hline & & $50+$ & 681 & 1,022 \\
\hline & \multirow{2}{*}{ Technical Support } & $<50$ & 398 & 962 \\
\hline & & $50+$ & 500 & 1,271 \\
\hline & \multirow{2}{*}{ Service } & $<50$ & 132 & 692 \\
\hline & & $50+$ & 277 & 0 \\
\hline & \multirow{2}{*}{ Security and Fire } & $<50$ & 429 & 0 \\
\hline & & $50+$ & 0 & 0 \\
\hline & \multirow{2}{*}{ Crafts } & $<50$ & 216 & 447 \\
\hline & & $50+$ & 232 & 125 \\
\hline & \multirow{2}{*}{ Line Operators } & $<50$ & 861 & 1,798 \\
\hline & & $50+$ & 1,197 & 2,043 \\
\hline
\end{tabular}

Figure 12. Rates for Selected Diagnostic Categories by Job Category, Gender, and Age

\begin{tabular}{|c|c|c|c|c|}
\hline \multirow{2}{*}{ Cancer } & \multicolumn{4}{|c|}{ Rate per 1,000} \\
\hline & Job Category & Age & Men & Women \\
\hline & Professional & $<50$ & 8 & 4 \\
\hline & & $50+$ & 17 & 37 \\
\hline & Administrative Sunport & $<50$ & 0 & 37 \\
\hline & & $50+$ & 88 & 62 \\
\hline & Technical Support & $<50$ & 1 & 3 \\
\hline & & $50+$ & 9 & 19 \\
\hline & Service & $<50$ & 0 & 77 \\
\hline & 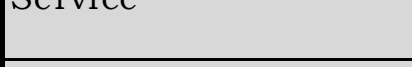 & $50+$ & 4 & 0 \\
\hline & Security and Fire & $<50$ & 0 & 0 \\
\hline & 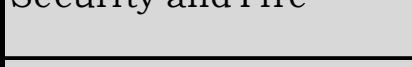 & $50+$ & 0 & 0 \\
\hline & Crafts & $<50$ & 6 & 0 \\
\hline & & $50+$ & 3 & 0 \\
\hline & Line $\mathrm{Or}$ & $<50$ & 4 & 20 \\
\hline & & $50+$ & 12 & 74 \\
\hline
\end{tabular}


Figure 12. Rates for Selected Diagnostic Categories by Job Category, Gender, and Age (Continued)

\begin{tabular}{|l|l|c|c|c|}
\hline \multirow{2}{*}{ Heart/Circulatory } & \multicolumn{4}{|c|}{ Rate per 1,000 } \\
\cline { 2 - 5 } & \multicolumn{1}{|c|}{ Job Category } & Age & M en & W omen \\
\cline { 2 - 5 } & Profe ssional & $<50$ & $\mathbf{1 5}$ & $\mathbf{2 5}$ \\
\cline { 2 - 5 } & $50+$ & $\mathbf{3 8}$ & $\mathbf{3 4}$ \\
\hline \multirow{2}{*}{ Administrative Support } & $<50$ & $\mathbf{7}$ & $\mathbf{1 4}$ \\
\cline { 2 - 5 } & $50+$ & $\mathbf{6 0}$ & $\mathbf{1 9}$ \\
\hline \multirow{3}{*}{ Technical Support } & $<50$ & $\mathbf{8}$ & $\mathbf{2 2}$ \\
\cline { 2 - 5 } & $50+$ & $\mathbf{5 7}$ & $\mathbf{3 8}$ \\
\cline { 2 - 5 } & \multirow{2}{*}{ Service } & $<50$ & $\mathbf{1 5}$ & $\mathbf{0}$ \\
\cline { 2 - 5 } & $50+$ & $\mathbf{4 0}$ & $\mathbf{0}$ \\
\cline { 2 - 5 } & \multirow{2}{*}{ Security and Fire } & $<50$ & $\mathbf{3 6}$ & $\mathbf{0}$ \\
\cline { 2 - 5 } & $50+$ & $\mathbf{0}$ & $\mathbf{0}$ \\
\cline { 2 - 5 } & \multirow{2}{*}{ Crafts } & $<50$ & $\mathbf{1 1}$ & $\mathbf{7 1}$ \\
\cline { 2 - 5 } & $50+$ & $\mathbf{3 7}$ & $\mathbf{0}$ \\
\cline { 2 - 5 } & \multirow{2}{*}{ Line Operators } & $<50$ & $\mathbf{2 3}$ & $\mathbf{7 1}$ \\
\cline { 2 - 5 } & $50+$ & $\mathbf{9 4}$ & $\mathbf{1 0 5}$ \\
\hline
\end{tabular}

\begin{tabular}{|c|c|c|c|c|}
\hline \multirow{2}{*}{ Respiratory } & \multicolumn{4}{|c|}{ Rate per 1,000} \\
\hline & Job Category & Age & Men & Women \\
\hline & \multirow{2}{*}{ Professional } & $<50$ & 105 & 105 \\
\hline & & $50+$ & 69 & 147 \\
\hline & \multirow{2}{*}{ Administrative Support } & $<50$ & 48 & 115 \\
\hline & & $50+$ & 93 & 179 \\
\hline & \multirow{2}{*}{ Technical Support } & $<50$ & 61 & 161 \\
\hline & & $50+$ & 92 & 259 \\
\hline & \multirow{2}{*}{ Service } & $<50$ & 29 & 0 \\
\hline & & $50+$ & 40 & 0 \\
\hline & \multirow{2}{*}{ Security and Fire } & $<50$ & 36 & 0 \\
\hline & & $50+$ & 0 & 0 \\
\hline & \multirow{2}{*}{ Crafts } & $<50$ & 26 & 59 \\
\hline & & $50+$ & 24 & 0 \\
\hline & \multirow{2}{*}{ Line Operators } & $<50$ & 200 & 384 \\
\hline & & $50+$ & 222 & 358 \\
\hline
\end{tabular}


Figure 12. Rates for Selected Diagnostic Categories by Job Category, Gender, and Age (Continued)

\begin{tabular}{|c|c|c|c|c|}
\hline \multirow{2}{*}{ Injury } & \multicolumn{4}{|c|}{ Rate per 1,000} \\
\hline & Job Category & Age & Men & Women \\
\hline & \multirow{2}{*}{ Professional } & $<50$ & 38 & 56 \\
\hline & & $50+$ & 40 & 48 \\
\hline & \multirow{2}{*}{ Administrative Support } & $<50$ & 69 & 41 \\
\hline & & $50+$ & 27 & 59 \\
\hline & \multirow{2}{*}{ Technical Support } & $<50$ & 36 & 76 \\
\hline & & $50+$ & 49 & 73 \\
\hline & \multirow{2}{*}{ Service } & $<50$ & 15 & 0 \\
\hline & & $50+$ & 31 & 0 \\
\hline & \multirow{2}{*}{ Security and Fire } & $<50$ & 36 & 0 \\
\hline & & $50+$ & 0 & 0 \\
\hline & \multirow{2}{*}{ Crafts } & $<50$ & 30 & 12 \\
\hline & & $50+$ & 8 & 42 \\
\hline & \multirow{2}{*}{ Line Operators } & $<50$ & 58 & 131 \\
\hline & & $50+$ & 65 & 123 \\
\hline
\end{tabular}

Time Trends

Figure 13. Age-Adjusted Rates for All Diagnoses Combined Among Women and Men from 2006 to 2010*

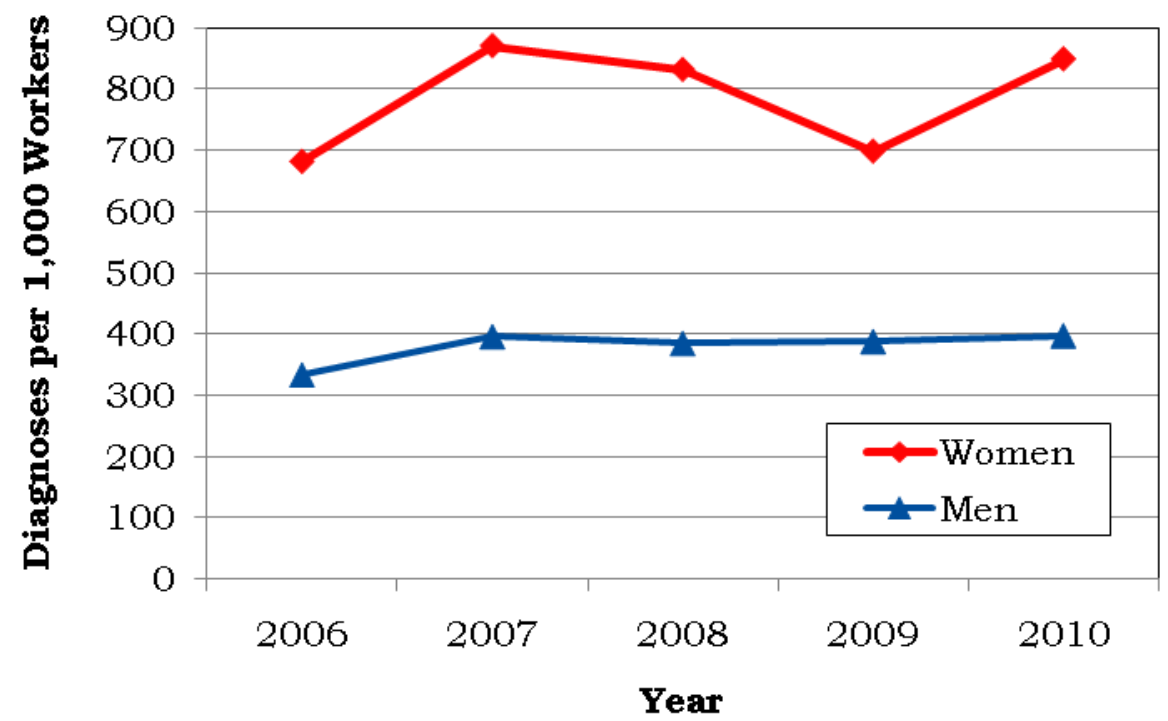

*Standardized to age distribution of 2000 U.S. population. 
Figure 14. Age-Adjusted Rates for Selected Diagnostic Categories Among Women and Men from 2006 to 2010*

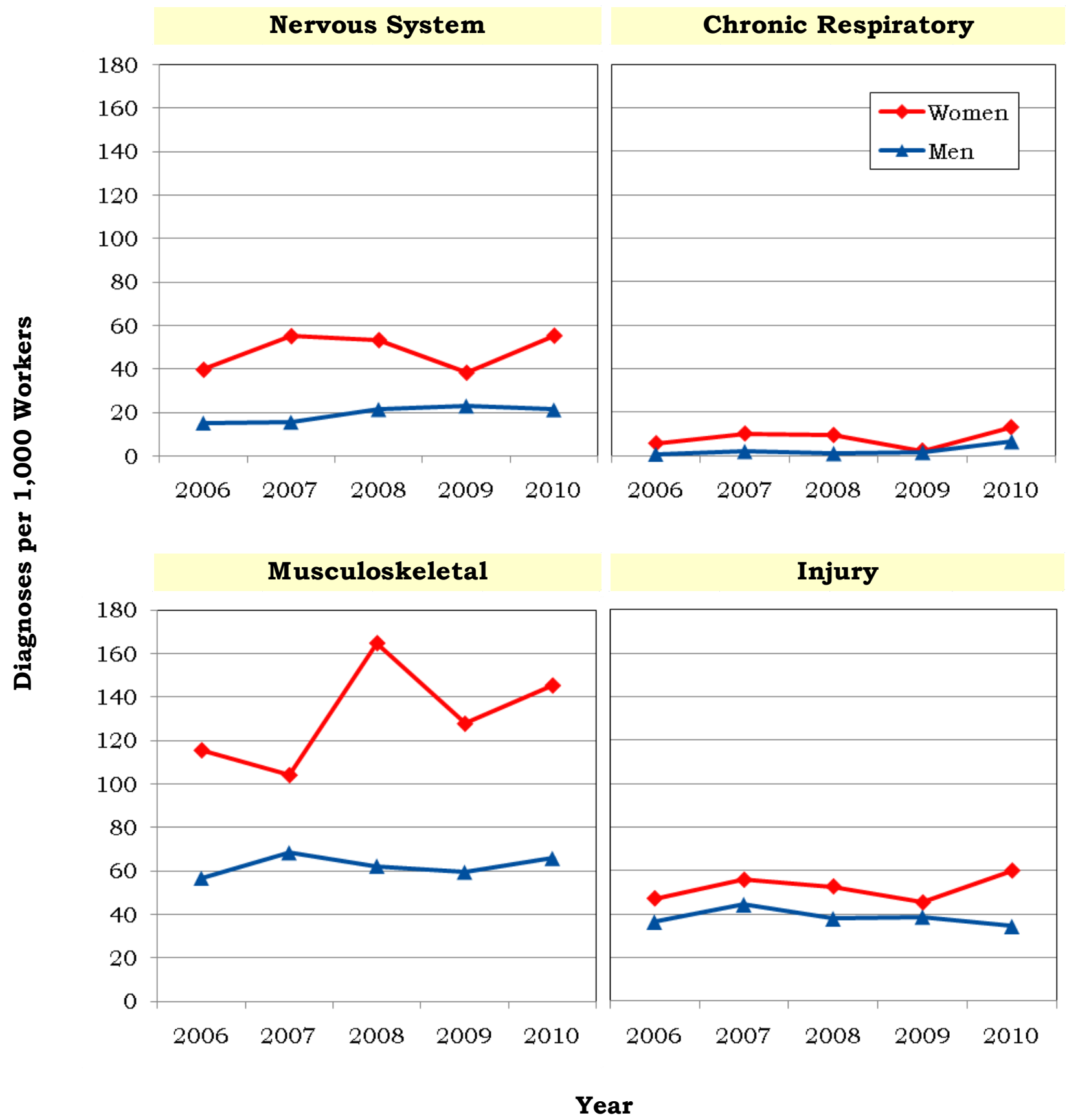

*Standardized to age distribution of 2000 U.S. population. 
Figure 15. Age-Adjusted Rates for All Diagnoses Combined Among Women and Men by Job Category from 2006 to 2010*

Professional

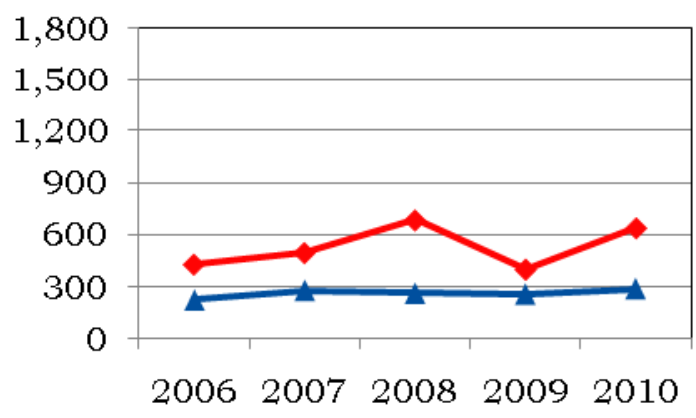

Administrative Support

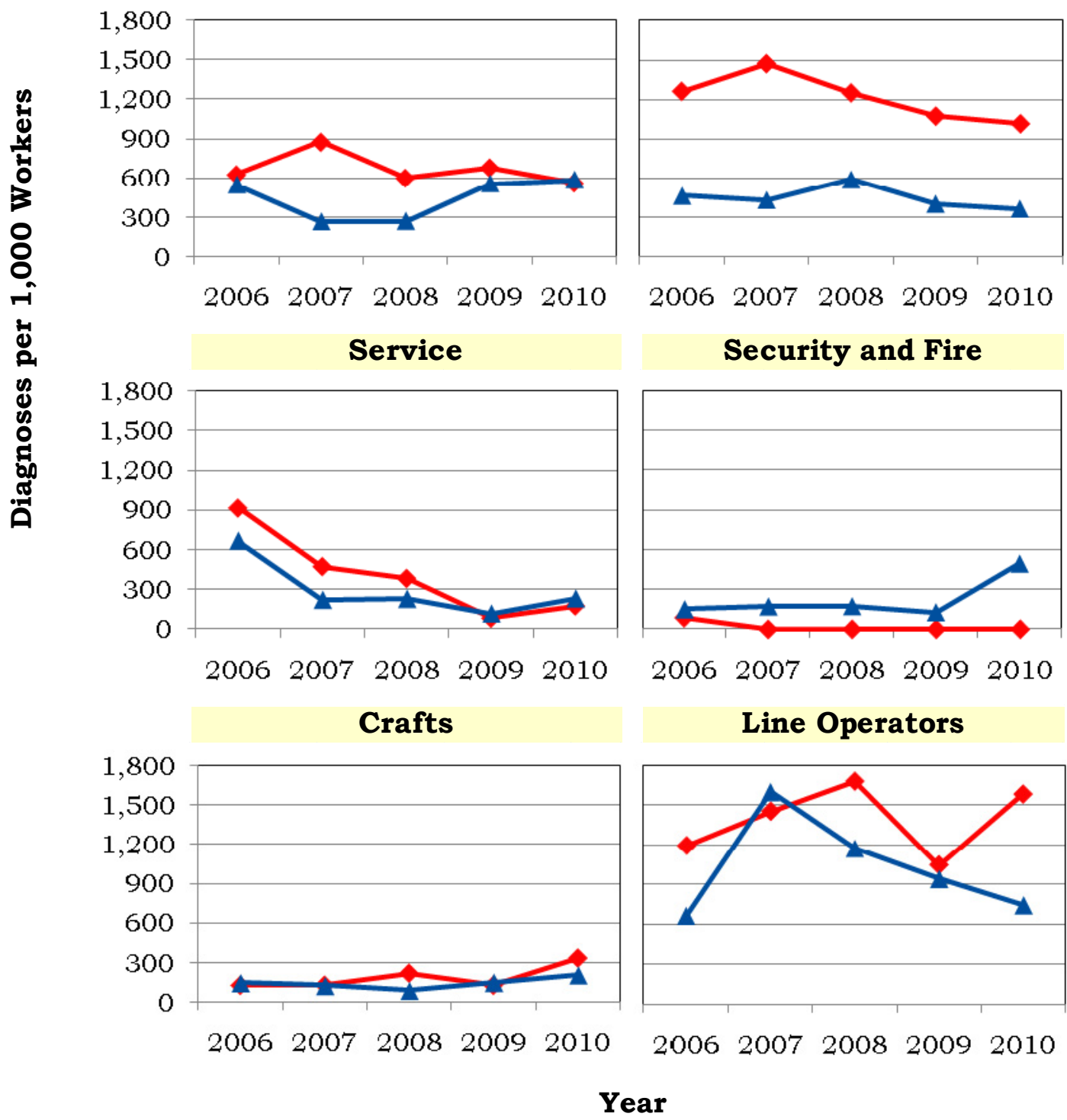

*Standardized to age distribution of 2000 U.S. population. 


\section{Sentinel Health Events for Occupations (SHEOs)}

An occupational sentinel health event (SHEO) is a disease, disability, or death that is likely to be occupationally related. Although sentinel health events may indicate an occupational exposure, many may result from nonoccupational exposures. Sentinel health events are therefore assessed in two categories:

Definite Sentinel Health Events: Diseases that are unlikely to occur in the absence of an occupational exposure (e.g., asbestosis).

Possible Sentinel Health Events: Diseases that may be occupational but can also occur in the absence of an occupational exposure (e.g., lung cancer or carpal tunnel syndrome).

Figure 16. Characteristics of SHEOs by Gender

\begin{tabular}{|l|c|c|c|c|}
\hline \multirow{2}{*}{} & \multicolumn{2}{|c|}{$\begin{array}{c}\text { Total Number of } \\
\text { SHEO Diagnoses }\end{array}$} & \multicolumn{2}{c|}{$\begin{array}{c}\text { Total Number of } \\
\text { Days Absent }\end{array}$} \\
\cline { 2 - 5 } & Men & Women & Men & W omen \\
\hline Definite & 5 & 7 & 13 & 182 \\
\hline Possible & 29 & 25 & 686 & 577 \\
\hline Total & 34 & 32 & 699 & 759 \\
\hline
\end{tabular}

Figure 17. SHEO Diagnoses by Gender

\begin{tabular}{|l|c|c|}
\hline \multirow{2}{*}{ Diagnoses } & \multicolumn{2}{c|}{ Gender } \\
\cline { 2 - 3 } & W omen & M en \\
\hline Carpal Tunnel Syndrome & 18 & 14 \\
\hline Pneumoconiosis (Berylliosis) & 1 & 2 \\
\hline M usculoskeletal Conditions & 2 & 1 \\
\hline Injuries & 3 & 1 \\
\hline Other Conditions & 8 & 16 \\
\hline
\end{tabular}


Occupational Safety and Health Administration (OSHA)-Recordable Events

Figure 18. OSHA-Recordable Events by Gender and Age

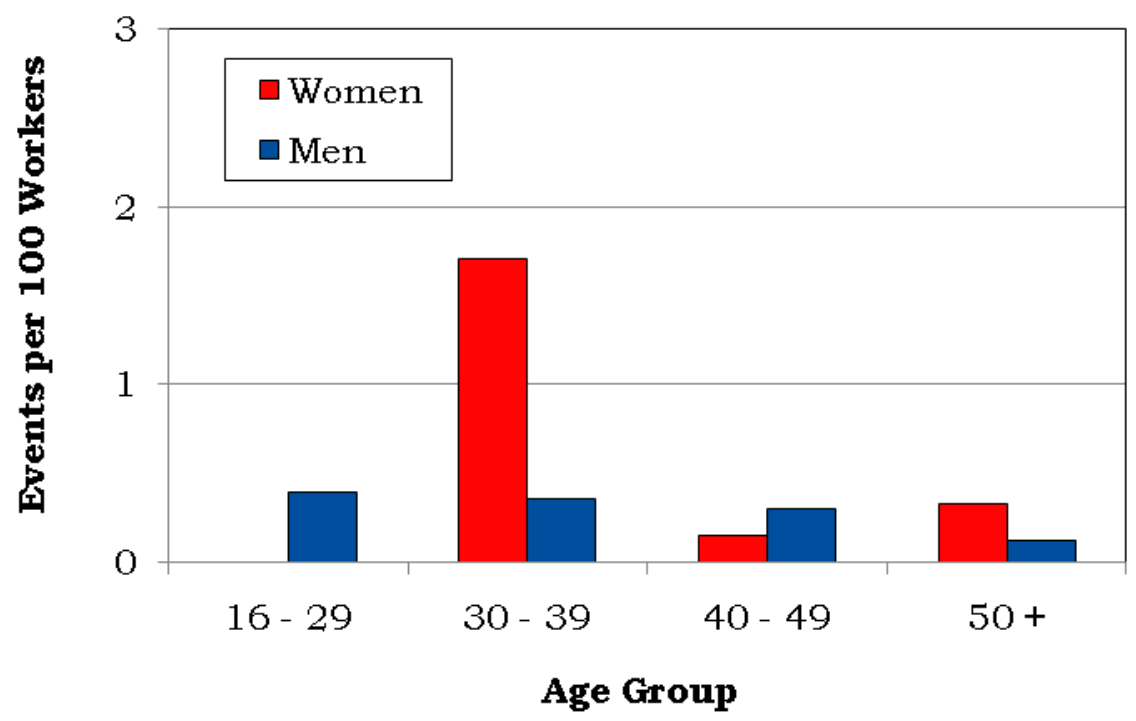

Figure 19. OSHA-Recordable Events by Job Category and Gender

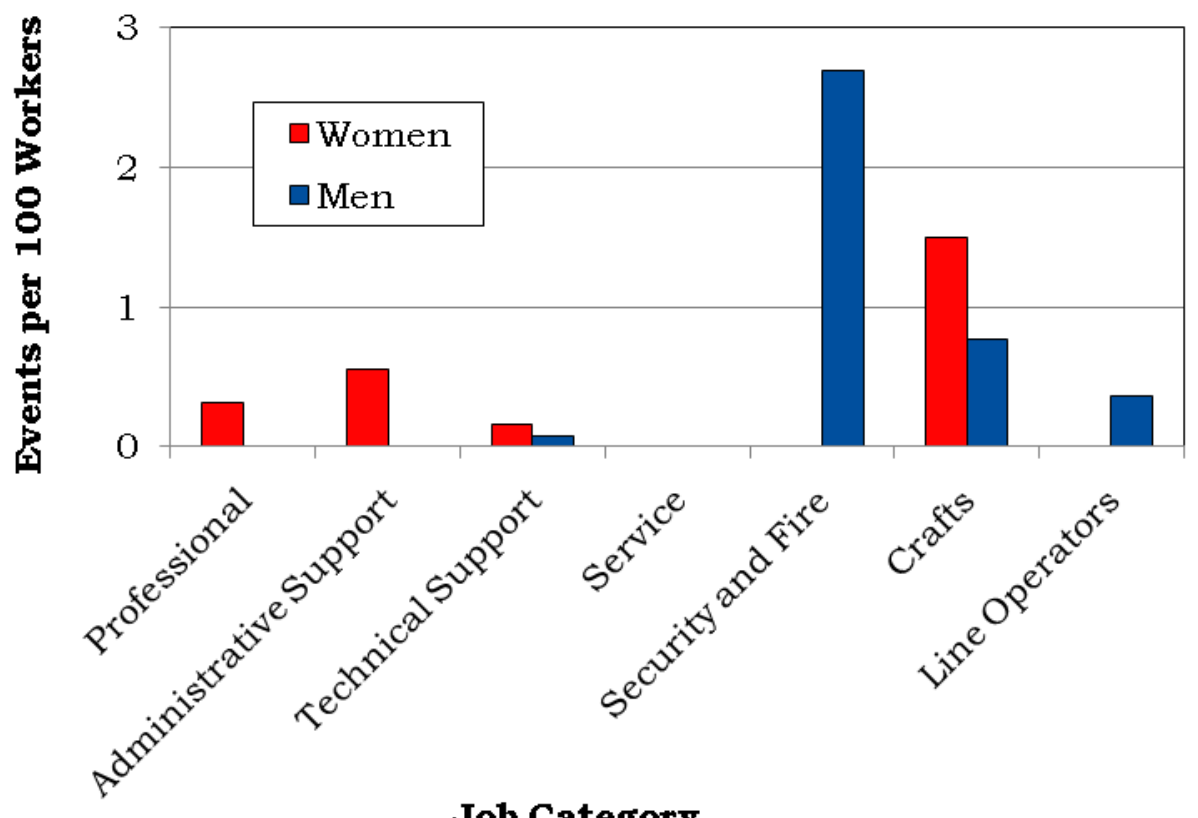

Job Category 


\section{Diagnostic and Accident Categories for OSHA-Recordable Events}

Figure 20. OSHA-Recordable Diagnoses by Diagnostic Category and Gender

\begin{tabular}{|l|c|c|}
\hline \multirow{2}{*}{ Diagnostic Category } & \multicolumn{2}{c|}{ Gender } \\
\cline { 2 - 3 } & Women & M en \\
\hline Musculoskeletal & $\mathbf{1}$ & $\mathbf{0}$ \\
\hline Nervous System & $\mathbf{0}$ & $\mathbf{1}$ \\
\hline Unspecified Symptoms & $\mathbf{1}$ & $\mathbf{0}$ \\
\hline Injury & $\mathbf{0}$ & $\mathbf{1 7}$ \\
\hline Fractures - Upper Limb & $\mathbf{2}$ & $\mathbf{2}$ \\
\hline Dislocations & $\mathbf{2}$ & $\mathbf{2}$ \\
\hline Back Sprains \& Strains & $\mathbf{3}$ & $\mathbf{1}$ \\
\hline Other Sprains \& Strains & $\mathbf{0}$ & $\mathbf{6}$ \\
\hline Open Wounds - Upper Limb & $\mathbf{2}$ & $\mathbf{0}$ \\
\hline Superficial Injuries & $\mathbf{3}$ & $\mathbf{1}$ \\
\hline Bruises & $\mathbf{0}$ & $\mathbf{1}$ \\
\hline Foreign Bodies Entering Orifice & $\mathbf{0}$ & $\mathbf{1}$ \\
\hline Burns & $\mathbf{0}$ & $\mathbf{2}$ \\
\hline Adverse Reactions to Non-Medical Substances & $\mathbf{1}$ & $\mathbf{1}$ \\
\hline Adverse Reactions to External Causes & &
\end{tabular}


Figure 21. OSHA-Recordable Accidents by Type and Gender

\begin{tabular}{|l|c|c|}
\hline \multirow{2}{*}{ Accident Category } & \multicolumn{2}{c|}{ Gender } \\
\cline { 2 - 3 } & Women & M en \\
\cline { 2 - 3 } & $\begin{array}{c}\text { Number of } \\
\text { Accidents }\end{array}$ & $\begin{array}{c}\text { Number of } \\
\text { Accidents }\end{array}$ \\
\hline Poisoning - Non-Medicinal & $\mathbf{0}$ & $\mathbf{1}$ \\
\hline Falls & $\mathbf{2}$ & $\mathbf{0}$ \\
\hline Natural/Environmental Factors & $\mathbf{1}$ & $\mathbf{2}$ \\
\hline Submersion/Suffocation/Foreign Bodies & $\mathbf{0}$ & $\mathbf{1}$ \\
\hline Other Accidents & $\mathbf{4}$ & $\mathbf{1 1}$ \\
\hline Struck by an Object & $\mathbf{2}$ & $\mathbf{0}$ \\
\hline Caught Between Objects & $\mathbf{0}$ & $\mathbf{2}$ \\
\hline Machinery & $\mathbf{0}$ & $\mathbf{1}$ \\
\hline Cutting/Piercing Instrument/Object & $\mathbf{0}$ & $\mathbf{4}$ \\
\hline Hot, Corrosive, or Caustic Material/Steam & $\mathbf{0}$ & $\mathbf{1}$ \\
\hline Overexertion/Strenuous Movements & $\mathbf{2}$ & $\mathbf{3}$ \\
\hline Total & $\mathbf{7}$ & $\mathbf{1 5}$ \\
\hline
\end{tabular}




\section{Rates of OSHA-Recordable Events}

Figure 22. OSHA-Recordable Rates by Age and Job Categories Among Women, All Diagnoses Combined

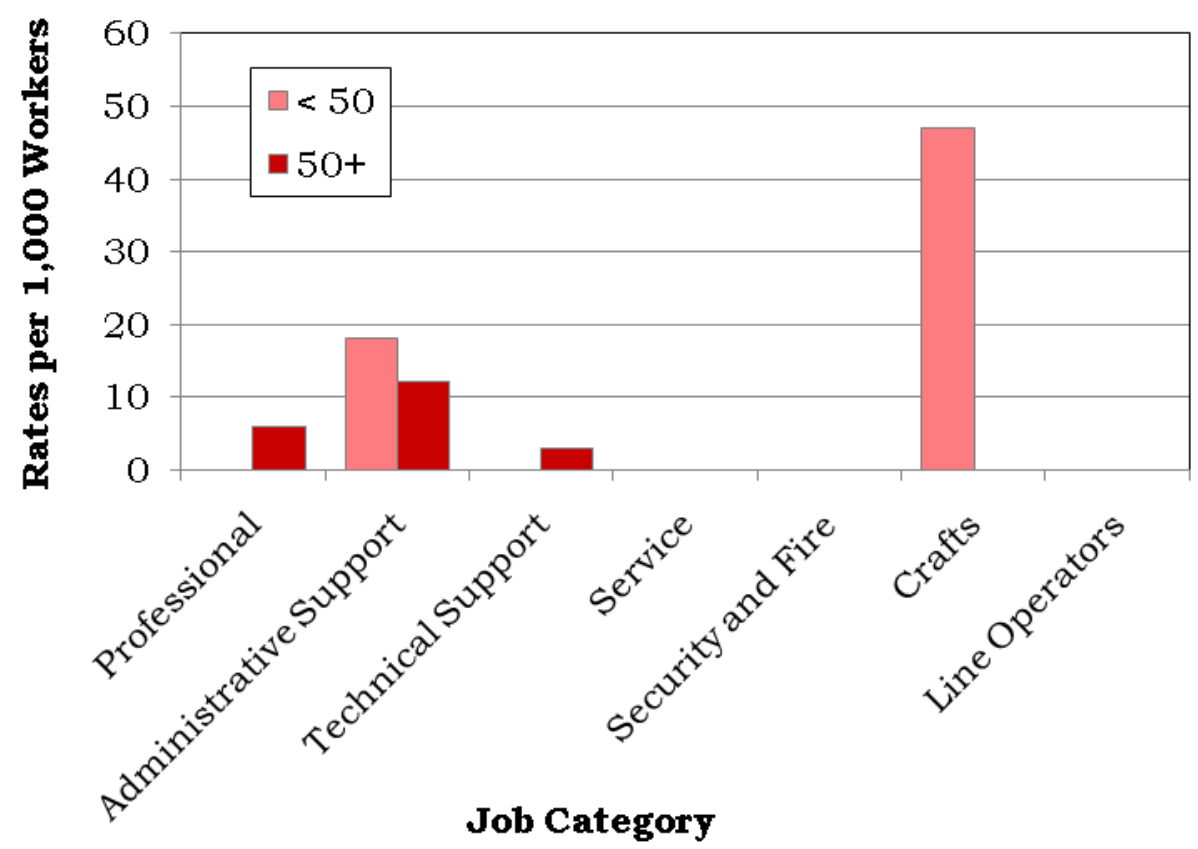

Figure 23. OSHA-Recordable Rates by Age and Job Categories Among Men, All Diagnoses Combined

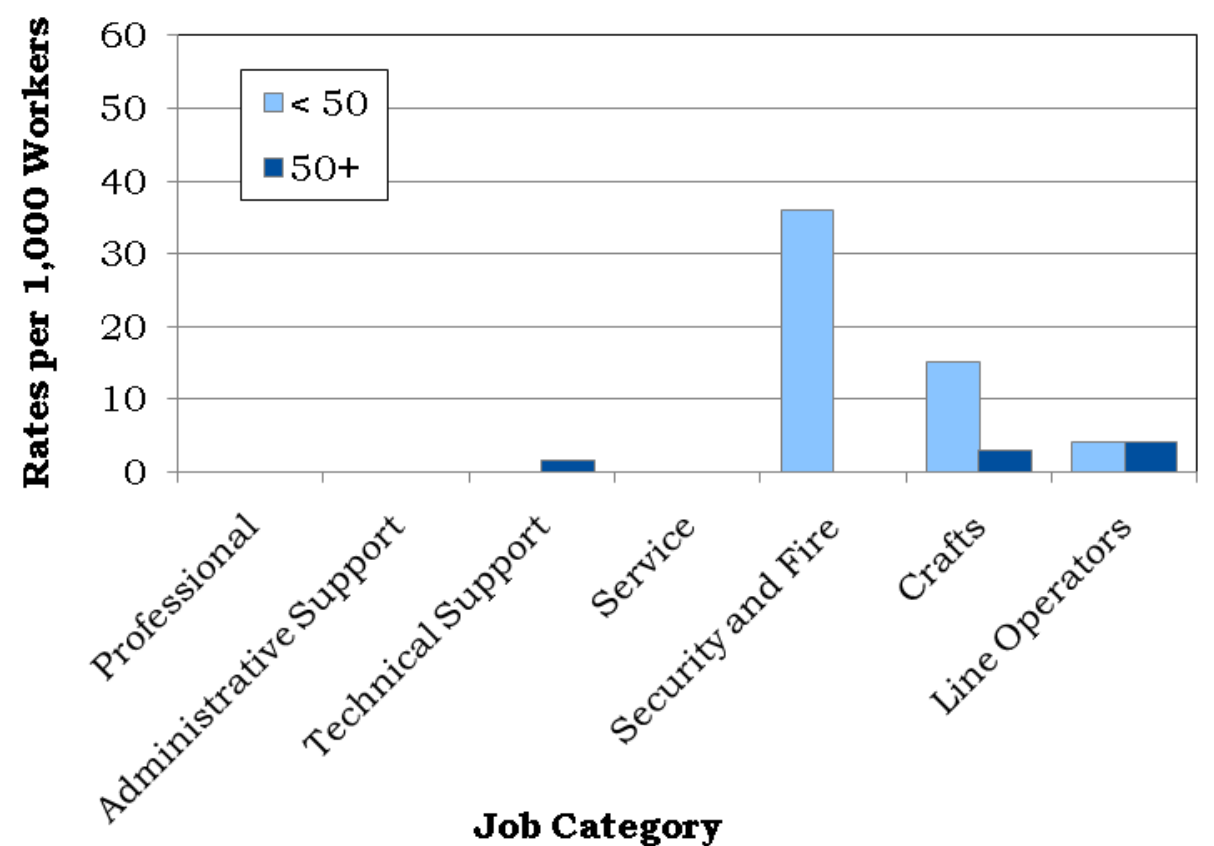

Job Category 


\section{Time Trends for OSHA-Recordable Events}

Figure 24. Age-Adjusted Rates for All OSHA-Recordable Diagnoses Combined Among Women and Men by Job Category from 2006 to 2010*

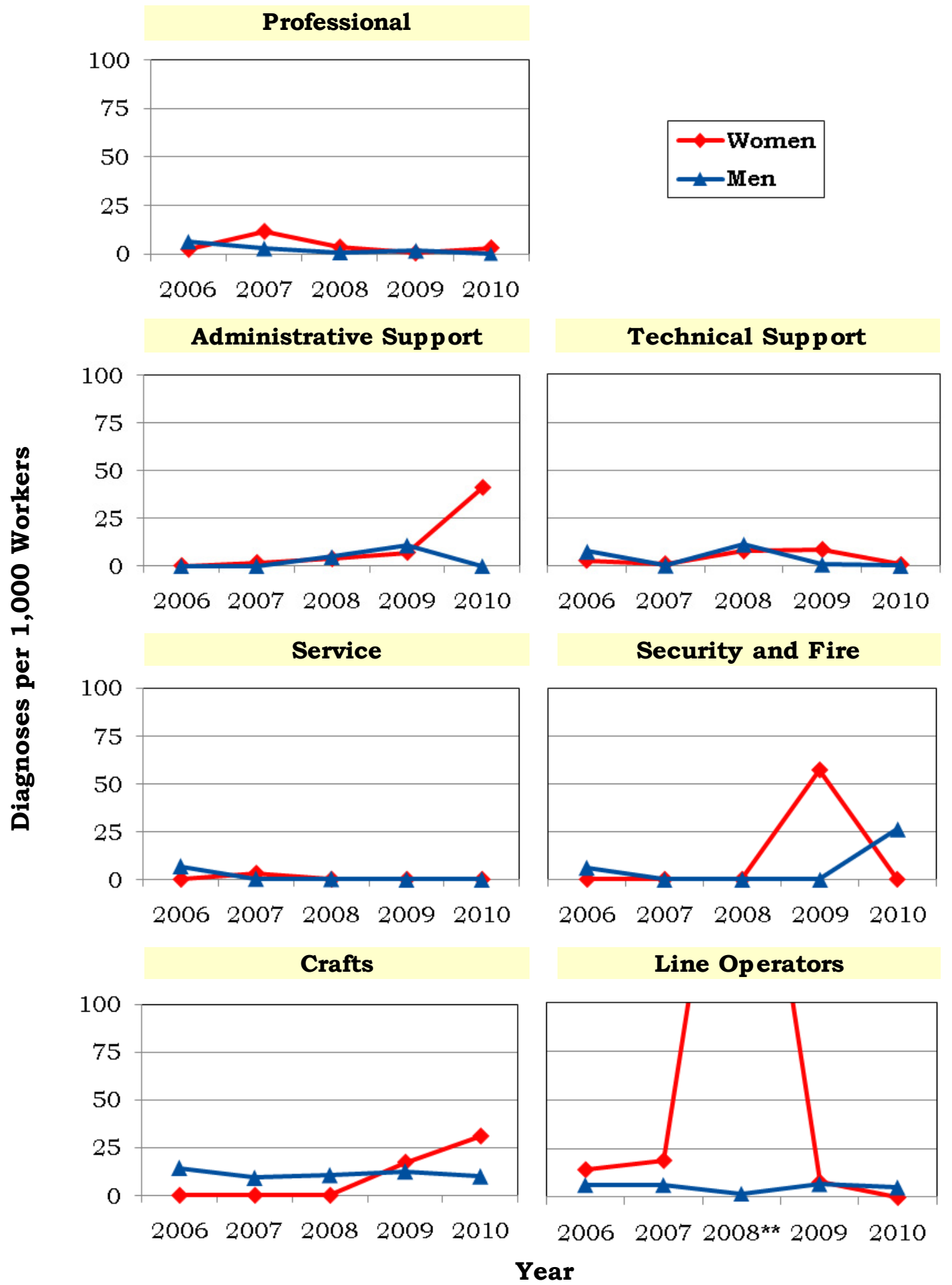

*Standardized to age distribution of 2000 U.S. population.

**The rate for female Line Operators for 2008 was truncated to 100 for graphical presentation. The actual rate was 595.83 diagnoses per 1,000 workers. 
Appendices 
Savannah River Site 2010

Absence Data

Appendix A. Work Force by Gender, Age, and Job Category

\begin{tabular}{|c|c|c|c|c|c|c|c|c|c|c|c|}
\hline \multirow{3}{*}{ Job Category } & \multicolumn{5}{|c|}{ Women } & \multicolumn{5}{|c|}{ Men } & \multirow[b]{3}{*}{ TOTAL } \\
\hline & \multicolumn{4}{|c|}{ Age Group } & \multirow[b]{2}{*}{ TOTAL } & \multicolumn{4}{|c|}{ Age Group } & \multirow[b]{2}{*}{ TOTAL } & \\
\hline & $16-29$ & $30-39$ & $40-49$ & $50+$ & & $16-29$ & $30-39$ & 40 - 49 & $50+$ & & \\
\hline Professional & 28 & 47 & 210 & 353 & 638 & 32 & 88 & 544 & 1,409 & 2,073 & 2,711 \\
\hline Administrative Support & 49 & 15 & 154 & 324 & 542 & 49 & 26 & 70 & 182 & 327 & 869 \\
\hline Technical Support & 49 & 66 & 201 & 317 & 633 & 203 & 165 & 560 & 1,317 & 2,245 & 2,878 \\
\hline Service & 0 & 1 & 12 & 5 & 18 & 0 & 2 & 66 & 224 & 292 & 310 \\
\hline Security and Fire & 0 & 0 & 0 & 3 & 3 & 4 & 9 & 15 & 9 & 37 & 40 \\
\hline Crafts & 19 & 30 & 36 & 48 & 133 & 164 & 210 & 289 & 380 & 1,043 & 1,176 \\
\hline Line Operators & 14 & 16 & 69 & 162 & 261 & 63 & 63 & 429 & 821 & 1,376 & 1,637 \\
\hline TOTAL & 159 & 175 & 682 & 1,212 & 2,228 & 515 & 563 & 1,973 & 4,342 & 7,393 & 9,621 \\
\hline
\end{tabular}

Appendix B. Age Distribution of the Work Force by Gender

\begin{tabular}{|l|r|r|r|r|r|r|r|r|}
\hline \multirow{3}{*}{ Year } & \multicolumn{9}{|c|}{ Women } & \multicolumn{4}{c|}{ Men } \\
\cline { 2 - 9 } & \multicolumn{1}{|c|}{ Percent Distribution by Age Group } & \multicolumn{1}{c|}{ Percent Distribution by Age Group } \\
\cline { 2 - 10 } & $\mathbf{1 6}-\mathbf{2 9}$ & $\mathbf{3 0}-\mathbf{3 9}$ & $\mathbf{4 0}-\mathbf{4 9}$ & $\mathbf{5 0}+$ & $\mathbf{1 6}-\mathbf{2 9}$ & $\mathbf{3 0}-\mathbf{3 9}$ & $\mathbf{4 0}-\mathbf{4 9}$ & $\mathbf{5 0}+$ \\
\hline $\mathbf{1 9 9 4}$ & 23.92 & 41.93 & 26.45 & 7.71 & 16.20 & 37.97 & 31.48 & 14.35 \\
\hline $\mathbf{1 9 9 5}$ & 18.31 & 42.58 & 29.86 & 9.25 & 11.66 & 37.77 & 34.26 & 16.31 \\
\hline $\mathbf{1 9 9 6}$ & 12.75 & 41.43 & 34.53 & 11.29 & 7.89 & 35.53 & 38.82 & 17.76 \\
\hline $\mathbf{1 9 9 7}$ & 9.53 & 38.76 & 38.48 & 13.23 & 5.51 & 33.40 & 41.02 & 20.06 \\
\hline $\mathbf{1 9 9 8}$ & 8.59 & 35.79 & 40.45 & 15.17 & 4.72 & 30.95 & 42.36 & 21.97 \\
\hline $\mathbf{1 9 9 9}$ & 6.30 & 33.07 & 42.76 & 17.87 & 3.87 & 28.08 & 42.83 & 25.22 \\
\hline $\mathbf{2 0 0 0}$ & 5.49 & 30.09 & 43.31 & 21.10 & 4.19 & 24.74 & 42.87 & 28.20 \\
\hline $\mathbf{2 0 0 1}$ & 5.97 & 26.63 & 43.88 & 23.52 & 4.86 & 22.01 & 43.32 & 29.81 \\
\hline $\mathbf{2 0 0 2}$ & 6.16 & 23.32 & 44.12 & 26.40 & 5.14 & 18.68 & 42.78 & 33.41 \\
\hline $\mathbf{2 0 0 3}$ & 5.22 & 16.88 & 50.33 & 27.57 & 4.93 & 13.71 & 46.65 & 34.70 \\
\hline $\mathbf{2 0 0 4}$ & 4.57 & 14.05 & 49.66 & 31.72 & 4.27 & 11.08 & 45.70 & 38.94 \\
\hline $\mathbf{2 0 0 5}$ & 3.22 & 11.65 & 44.16 & 40.98 & 3.03 & 9.45 & 39.27 & 48.25 \\
\hline $\mathbf{2 0 0 6}$ & 2.62 & 9.82 & 45.53 & 42.04 & 2.74 & 8.71 & 39.43 & 49.13 \\
\hline $\mathbf{2 0 0 7}$ & 5.22 & 7.33 & 47.81 & 39.63 & 4.02 & 6.37 & 42.47 & 47.13 \\
\hline $\mathbf{2 0 0 8}$ & 6.12 & 6.34 & 42.97 & 44.57 & 4.27 & 5.79 & 37.76 & 52.18 \\
\hline $\mathbf{2 0 0 9}$ & 7.27 & 7.87 & 33.22 & 51.65 & 5.30 & 6.79 & 29.56 & 58.35 \\
\hline $\mathbf{2 0 1 0}$ & 7.14 & 7.85 & 30.61 & 54.40 & 6.97 & 7.62 & 26.69 & 58.73 \\
\hline
\end{tabular}


Savannah River Site 2010

Absence Data

Appendix C. Total Number of Workers Who Reported at Least One Absence by Gender, Age, and Job Category*

\begin{tabular}{|c|c|c|c|c|c|c|c|c|c|c|c|}
\hline \multirow{3}{*}{ Job Category } & \multicolumn{5}{|c|}{ Women } & \multicolumn{5}{|c|}{ Men } & \multirow[b]{3}{*}{ TOTAL } \\
\hline & \multicolumn{4}{|c|}{ Age Group } & \multirow[b]{2}{*}{ TOTAL } & \multicolumn{4}{|c|}{ Age Group } & \multirow[b]{2}{*}{ TOTAL } & \\
\hline & $16-29$ & 30 - 39 & $40-49$ & $50+$ & & $16-29$ & 30 - 39 & $40-49$ & $50+$ & & \\
\hline Professional & 2 & 9 & 50 & 77 & 138 & 3 & 6 & 100 & 241 & 350 & 488 \\
\hline Administrative Support & 6 & 0 & 40 & 110 & 156 & 3 & 5 & 9 & 39 & 56 & 212 \\
\hline Technical Support & 7 & 18 & 62 & 109 & 196 & 11 & 23 & 68 & 256 & 358 & 554 \\
\hline Service & 0 & 0 & 3 & 0 & 3 & 0 & 1 & 4 & 26 & 31 & 34 \\
\hline Security and Fire & 0 & 0 & 0 & 0 & 0 & 2 & 1 & 1 & 0 & 4 & 4 \\
\hline Crafts & 4 & 4 & 9 & 4 & 21 & 15 & 22 & 40 & 43 & 120 & 141 \\
\hline Line Operators & 6 & 7 & 35 & 80 & 128 & 6 & 14 & 137 & 303 & 460 & 588 \\
\hline TOTAL & 25 & 38 & 199 & 380 & 642 & 40 & 72 & 359 & 908 & 1,379 & 2,021 \\
\hline
\end{tabular}

*Only those job categories and gender/age combinations with at least one absence appear in this table.

Appendix D. Total Number of Absences by Gender, Age, and Job Category*

\begin{tabular}{|c|c|c|c|c|c|c|c|c|c|c|c|}
\hline \multirow{3}{*}{ Job Category } & \multicolumn{5}{|c|}{ Women } & \multicolumn{5}{|c|}{ Men } & \multirow[b]{3}{*}{ TOTAL } \\
\hline & \multicolumn{4}{|c|}{ Age Group } & \multirow[b]{2}{*}{ TOTAL } & \multicolumn{4}{|c|}{ Age Group } & \multirow[b]{2}{*}{ TOTAL } & \\
\hline & $16-29$ & 30 - 39 & $40-49$ & $50+$ & & $16-29$ & $30-39$ & $40-49$ & $50+$ & & \\
\hline Professional & 4 & 11 & 75 & 114 & 204 & 3 & 7 & 139 & 344 & 493 & 697 \\
\hline Administrative Support & 10 & 0 & 61 & 168 & 239 & 4 & 6 & 31 & 61 & 102 & 341 \\
\hline Technical Support & 9 & 46 & 100 & 197 & 352 & 17 & 27 & 127 & 366 & 537 & 889 \\
\hline Service & 0 & 0 & 5 & 0 & 5 & 0 & 1 & 6 & 34 & 41 & 46 \\
\hline Security and Fire & 0 & 0 & 0 & 0 & 0 & 3 & 1 & 1 & 0 & 5 & 5 \\
\hline Crafts & 5 & 5 & 12 & 4 & 26 & 16 & 24 & 49 & 58 & 147 & 173 \\
\hline Line Operators & 8 & 11 & 72 & 158 & 249 & 8 & 18 & 228 & 498 & 752 & 1,001 \\
\hline TOTAL & 36 & 73 & 325 & 641 & 1,075 & 51 & 84 & 581 & 1,361 & 2,077 & 3,152 \\
\hline
\end{tabular}

*Only those job categories and gender/age combinations with at least one absence appear in this table. 
Savannah River Site 2010

Absence Data

Appendix E. Distribution of the Number of Calendar Days Missed per Absence by Gender and Age*

\begin{tabular}{|c|c|c|c|c|c|c|c|c|c|c|c|}
\hline \multirow{3}{*}{$\begin{array}{c}\text { Number of } \\
\text { Calendar Days }\end{array}$} & \multicolumn{5}{|c|}{ Women } & \multicolumn{5}{|c|}{ Men } & \multirow[b]{3}{*}{ TOTAL } \\
\hline & \multicolumn{4}{|c|}{ Age Group } & \multirow[b]{2}{*}{ TOTAL } & \multicolumn{4}{|c|}{ Age Group } & \multirow[b]{2}{*}{ TOTAL } & \\
\hline & $16-29$ & $30-39$ & $40-49$ & $50+$ & & $16-29$ & $30-39$ & $40-49$ & $50+$ & & \\
\hline$<15$ & 33 & 58 & 251 & 480 & 822 & 44 & 71 & 454 & 1,013 & 1,582 & 2,404 \\
\hline $15-28$ & 0 & 9 & 38 & 81 & 128 & 3 & 6 & 76 & 171 & 256 & 384 \\
\hline $29-42$ & 0 & 1 & 15 & 26 & 42 & 3 & 4 & 18 & 67 & 92 & 134 \\
\hline $43-56$ & 3 & 2 & 8 & 19 & 32 & 0 & 0 & 11 & 42 & 53 & 85 \\
\hline $57-91$ & 0 & 3 & 8 & 25 & 36 & 1 & 2 & 15 & 47 & 65 & 101 \\
\hline $92-182$ & 0 & 0 & 5 & 8 & 13 & 0 & 1 & 6 & 18 & 25 & 38 \\
\hline $183+$ & 0 & 0 & 0 & 2 & 2 & 0 & 0 & 1 & 3 & 4 & 6 \\
\hline TOTAL & 36 & 73 & 325 & 641 & 1,075 & 51 & 84 & 581 & 1,361 & 2,077 & 3,152 \\
\hline
\end{tabular}

*Only those gender/age combinations with at least one absence appear in this table. 
Savannah River Site 2010

Absence Data

Appendix F. Distribution of the Number of Calendar Days Missed per Absence by Gender and Job Category*

Women

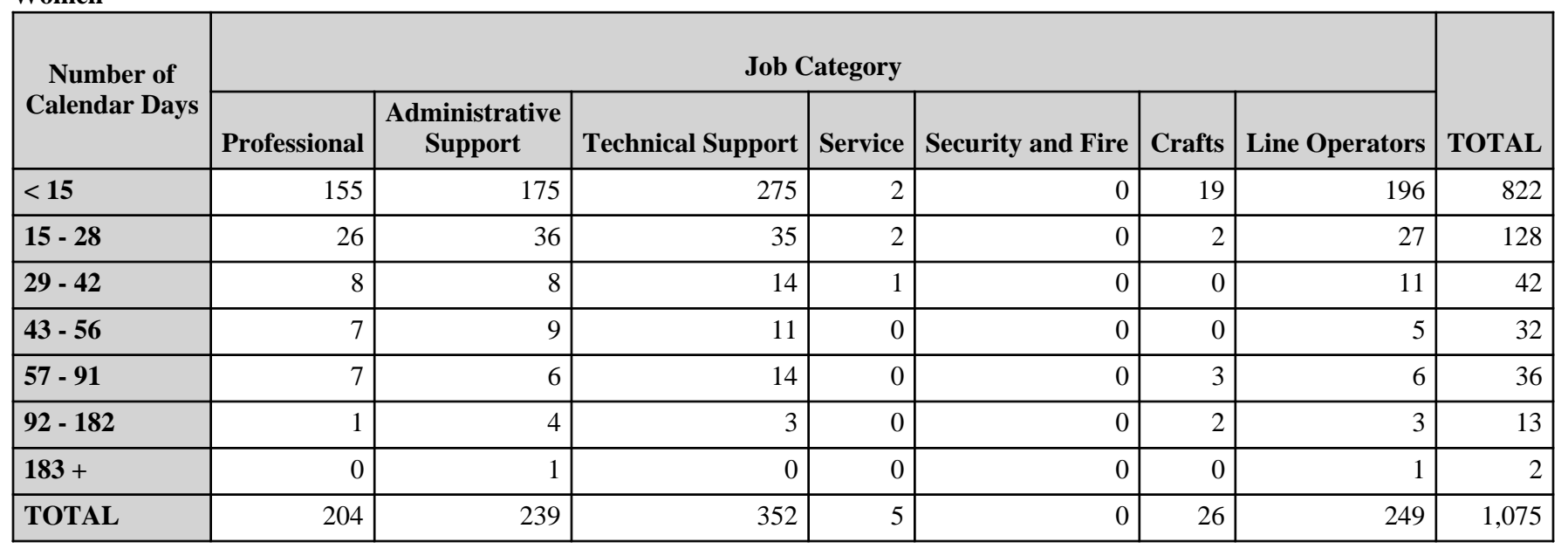

Men

\begin{tabular}{|c|c|c|c|c|c|c|c|c|}
\hline \multirow{2}{*}{$\begin{array}{c}\text { Number of } \\
\text { Calendar Days }\end{array}$} & \multicolumn{7}{|c|}{ Job Category } & \multirow[b]{2}{*}{ TOTAL } \\
\hline & Professional & $\begin{array}{c}\text { Administrative } \\
\text { Support }\end{array}$ & Technical Support & Service & Security and Fire & Crafts & Line Operators & \\
\hline$<15$ & 365 & 75 & 398 & 29 & 4 & 121 & 590 & 1,582 \\
\hline $15-28$ & 61 & 16 & 82 & 6 & 1 & 8 & 82 & 256 \\
\hline $57-91$ & 20 & 3 & 14 & 1 & 0 & 4 & 23 & 65 \\
\hline 92 - 182 & 10 & 2 & 5 & 0 & 0 & 2 & 6 & 25 \\
\hline $183+$ & 1 & 0 & 2 & 0 & 0 & 0 & 1 & 4 \\
\hline TOTAL & 493 & 102 & 537 & 41 & 5 & 147 & 752 & 2,077 \\
\hline
\end{tabular}

*Only those gender/job category combinations with at least one absence appear in this table. 


\section{Savannah River Site 2010}

\section{Absence Data}

Appendix G. Number of Diagnoses in Each Diagnostic Category by Gender and Age*

\begin{tabular}{|c|c|c|c|c|c|c|}
\hline & & \multicolumn{5}{|c|}{ Women } \\
\hline & & \multicolumn{4}{|c|}{ Age Group } & \multirow[b]{2}{*}{ TOTAL } \\
\hline & & $16-29$ & $30-39$ & $40-49$ & $50+$ & \\
\hline Diagnostic Category & ICD-9-CM Code & \multirow[b]{2}{*}{2} & \multirow[b]{2}{*}{3} & \multirow[b]{2}{*}{23} & \multirow[b]{2}{*}{41} & \multirow[b]{2}{*}{69} \\
\hline INFECTIOUS \& PARASITIC DISEASES (DIS) & 001-139 & & & & & \\
\hline -Intestinal Infectious Dis & 001-009 & 2 & 2 & 12 & 13 & 29 \\
\hline -Other Bacterial Dis & 030-041 & 0 & 0 & 4 & 11 & 15 \\
\hline -HIV & 042 & 0 & 0 & 0 & 0 & 0 \\
\hline -Viral Dis with Exanthem & \begin{tabular}{|c|c|}
$050-059$ \\
\end{tabular} & 0 & 0 & 0 & 8 & 8 \\
\hline -Other Viral Dis \& Chlamydiae & 070-079 & 0 & 1 & 6 & 6 & 13 \\
\hline -Rickettsioses \& Other Arthropod & $080-088$ & 0 & 0 & 0 & 0 & 0 \\
\hline -Syphilis \& Other Venereal Dis & \begin{tabular}{|c|}
$090-099$ \\
\end{tabular} & 0 & 0 & 1 & 0 & 1 \\
\hline -Mycoses & 110-118 & 0 & 0 & 0 & 3 & 3 \\
\hline -Other Infections \& Parasitic Dis & $130-136$ & 0 & 0 & 0 & 0 & 0 \\
\hline MALIGNANT NEOPLASMS & $\begin{array}{l}140-208, \\
209.0-209.3 \\
230-234\end{array}$ & 0 & 0 & 13 & 51 & 64 \\
\hline -Lip, Oral Cavity, Pharynx & 140-149 & 0 & 0 & 0 & 2 & 2 \\
\hline -Digestive \& Peritoneal & 150-159 & 0 & 0 & 2 & 0 & 2 \\
\hline -Respiratory \& Intrathoracic & $160-165$ & 0 & 0 & 5 & 1 & $\overline{6}$ \\
\hline -Bone, Connective Tissue, Skin & $170-173,176$ & 0 & 0 & 1 & 2 & 3 \\
\hline -Breast & 174-175 & 0 & 0 & 4 & 22 & 26 \\
\hline -Genitourinary & 179-189 & 0 & 0 & 1 & 6 & 7 \\
\hline -Endocrine & $\begin{array}{l}\text { 193-194, } \\
\text { 209.0-209.3 }\end{array}$ & 0 & 0 & 0 & 2 & 2 \\
\hline -Other \& Unspecified Sites & $195-199,209.7$ & 0 & 0 & 0 & 6 & 6 \\
\hline -Lymphatic \& Hematopoietic & $200-208$ & 0 & 0 & 0 & 10 & 10 \\
\hline BENIGN \& UNCERTAIN NEOPLASMS & $\begin{array}{l}209.4-209.6 \\
210-229,235-239\end{array}$ & 2 & 5 & 16 & 19 & 42 \\
\hline ENDOCRINE/METABOLIC/IMMUNITY & $240-279$ & 0 & 1 & 15 & 30 & 46 \\
\hline -Thyroid Gland Disorders & $240-246$ & 0 & 1 & 2 & 4 & 7 \\
\hline -Other Endocrine Gland Dis & 249-259 & 0 & 0 & 4 & 15 & 19 \\
\hline -Other Metabolic \& Immunity Disorders & $270-279$ & 0 & 0 & 9 & 11 & 20 \\
\hline BLOOD \& BLOOD-FORMING ORGANS & $280-289$ & 0 & 2 & 8 & 13 & 23 \\
\hline MENTAL DISORDERS & 290-319 & 5 & 0 & 13 & 12 & 30 \\
\hline -Psychoses & $290-299$ & 1 & 0 & 3 & 2 & 6 \\
\hline -Non-Psychotic Disorders & $300-302,306-316$ & 4 & 0 & 9 & 8 & 21 \\
\hline -Alcohol Dependence & 303 & 0 & 0 & 0 & 0 & 0 \\
\hline -Drug Dependence & $304-305$ & 0 & 0 & 1 & 2 & 3 \\
\hline NERVOUS SYSTEM (NS) \& SENSE ORGANS & 320-389 & 4 & 12 & 26 & 90 & 132 \\
\hline -Inflammatory Dis of Central NS & $320-326$ & 0 & 0 & 0 & 0 & 0 \\
\hline -Organic Sleep Disorders & 327 & 0 & 0 & 0 & 0 & 0 \\
\hline
\end{tabular}

(Continued)

*Only those diagnostic categories and gender/age combinations with at least one occurrence appear in this table. 


\section{Savannah River Site 2010}

\section{Absence Data}

Appendix G. Number of Diagnoses in Each Diagnostic Category by Gender and Age*

\begin{tabular}{|c|c|c|c|c|c|c|c|}
\hline & & \multicolumn{5}{|c|}{ Men } & \multirow[b]{3}{*}{ TOTAL } \\
\hline & & \multicolumn{4}{|c|}{ Age Group } & \multirow[b]{2}{*}{ TOTAL } & \\
\hline & & $16-29$ & 30 - 39 & $40-49$ & $50+$ & & \\
\hline INFECTIOUS \& PARASITIC DISEASES (DIS) & 001-139 & 11 & 8 & 53 & 68 & 140 & 209 \\
\hline -Intestinal Infectious Dis & 001-009 & 7 & 4 & 28 & 39 & 78 & 107 \\
\hline -HIV & 042 & 0 & 0 & 1 & 0 & 1 & 1 \\
\hline -Viral Dis with Exanthem & 050-059 & 1 & 1 & 5 & 10 & 17 & 25 \\
\hline -Other Viral Dis \& Chlamydiae & 070-079 & 1 & 1 & 4 & 3 & 9 & 22 \\
\hline -Rickettsioses \& Other Arthropod & 080-088 & 0 & 0 & 0 & 1 & 1 & 1 \\
\hline MALIGNANT NEOPLASMS & $\begin{array}{l}\text { 140-208, } \\
209.0-209.3, \\
230-234\end{array}$ & 0 & 1 & 11 & 64 & 76 & 140 \\
\hline -Lip, Oral Cavity, Pharynx & $140-149$ & 0 & 0 & 1 & 4 & 5 & 7 \\
\hline -Digestive \& Peritoneal & 150-159 & 0 & 0 & 1 & 4 & 5 & 7 \\
\hline -Respiratory \& Intrathoracic & $160-165$ & 0 & 0 & 1 & 2 & 3 & 9 \\
\hline -Bone, Connective Tissue, Skin & $170-173,176$ & 0 & 0 & 5 & 13 & 18 & 21 \\
\hline -Breast & 174-175 & 0 & 0 & 0 & 0 & 0 & 26 \\
\hline ENDOCRINE/METABOLIC/IMMUNITY & $240-279$ & 1 & 3 & 53 & 75 & 132 & 178 \\
\hline -Thyroid Gland Disorders & $240-246$ & 0 & 1 & 9 & 4 & 14 & 21 \\
\hline -Other Endocrine Gland Dis & 249-259 & 0 & 0 & 21 & 24 & 45 & 64 \\
\hline -Other Metabolic \& Immunity Disorders & 270-279 & 1 & 2 & 23 & 47 & 73 & 93 \\
\hline BLOOD \& BLOOD-FORMING ORGANS & 280-289 & 0 & 0 & 12 & 17 & 29 & 52 \\
\hline MENTAL DISORDERS & 290-319 & 8 & 2 & 28 & 46 & 84 & 114 \\
\hline -Psychoses & 290-299 & 1 & 1 & 2 & 10 & 14 & 20 \\
\hline -Non-Psychotic Disorders & $300-302,306-316$ & 4 & 1 & 26 & 26 & 57 & 78 \\
\hline -Alcohol Dependence & 303 & 1 & 0 & 0 & 0 & 1 & 1 \\
\hline -Drug Dependence & $304-305$ & 2 & 0 & 0 & 10 & 12 & 15 \\
\hline NERVOUS SYSTEM (NS) \& SENSE ORGANS & 320-389 & 3 & 10 & 55 & 134 & 202 & 334 \\
\hline -Inflammatory Dis of Central NS & $320-326$ & 0 & 0 & 0 & 1 & 1 & 1 \\
\hline -Organic Sleep Disorders & 327 & 0 & 1 & 0 & 2 & 3 & 3 \\
\hline
\end{tabular}

(Continued)

*Only those diagnostic categories and gender/age combinations with at least one occurrence appear in this table. 


\section{Savannah River Site 2010}

\section{Absence Data}

Appendix G. Number of Diagnoses in Each Diagnostic Category by Gender and Age*

\begin{tabular}{|c|c|c|c|c|c|c|}
\hline & & \multicolumn{5}{|c|}{ Women } \\
\hline & & \multicolumn{4}{|c|}{ Age Group } & \multirow[b]{2}{*}{ TOTAL } \\
\hline & & $16-29$ & $30-39$ & $40-49$ & $\mathbf{5 0}+$ & \\
\hline Diagnostic Category & ICD-9-CM Code & \multirow[b]{2}{*}{0} & \multirow[b]{2}{*}{0} & \multirow[b]{2}{*}{0} & \multirow[b]{2}{*}{0} & \multirow[b]{2}{*}{0} \\
\hline -Hereditary/Degenerative Central NS Dis & 330-337 & & & & & \\
\hline -Pain & 338 & 0 & 1 & 0 & 0 & 1 \\
\hline -Other Headache Syndromes & 339 & 0 & 0 & 1 & 2 & 3 \\
\hline -Other Disorders of Central NS & $340-349$ & 2 & 8 & 11 & 16 & 37 \\
\hline -Disorders of Peripheral NS & $350-359$ & 0 & 2 & 4 & 24 & 30 \\
\hline -Disorders of Eye & $360-379$ & 2 & 0 & 3 & 38 & 43 \\
\hline -Diseases of Ear \& Mastoid & 380-389 & 0 & 1 & 7 & 10 & 18 \\
\hline CIRCULATORY SYSTEM & $390-459$ & 1 & 3 & 26 & 47 & 77 \\
\hline -Hypertensive Dis & $401-405$ & 0 & 1 & 5 & 15 & 21 \\
\hline -Ischemic Heart Dis & $410-414$ & 0 & 0 & 6 & 8 & 14 \\
\hline -Dis of Pulmonary Circulation & $415-417$ & 0 & 0 & 0 & 1 & 1 \\
\hline -Other Heart Dis & $420-429$ & 0 & 0 & 5 & 6 & 11 \\
\hline -Cerebrovascular Dis & $430-438$ & 0 & 0 & 1 & 4 & 5 \\
\hline -Dis of Arteries \& Capillaries & $440-449$ & 1 & 0 & 0 & 2 & 3 \\
\hline -Dis of Veins, Lymphatics, Other & $451-459$ & 0 & 2 & 9 & 11 & 22 \\
\hline RESPIRATORY SYSTEM & $460-519$ & 12 & 22 & 115 & 250 & 399 \\
\hline -Acute Respiratory Infections & $460-466$ & 8 & 15 & 64 & 152 & 239 \\
\hline -Other Dis Upper Respiratory Tract & $470-478$ & 2 & 4 & 25 & 36 & 67 \\
\hline -Pneumonia \& Influenza & $480-488$ & 2 & 0 & 16 & 34 & 52 \\
\hline -Chronic Obstructive Dis & $490-496$ & 0 & 3 & 6 & 23 & 32 \\
\hline -Lung Dis from External Agents & $500-508$ & 0 & 0 & 0 & 1 & 1 \\
\hline -Other Respiratory Dis & $510-519$ & 0 & 0 & 4 & 4 & 8 \\
\hline DIGESTIVE SYSTEM & $520-579$ & 9 & 10 & 43 & 68 & 130 \\
\hline -Oral Cavity, Saliva Glands, Jaw & $520-529$ & 5 & 6 & 7 & 12 & 30 \\
\hline -Esophagus, Stomach, Duodenum & $530-538$ & 2 & 0 & 8 & 17 & 27 \\
\hline -Appendicitis & $540-543$ & 0 & 0 & 1 & 3 & 4 \\
\hline -Hernias & $550-553$ & 1 & 0 & 8 & 7 & 16 \\
\hline -Enteritis, Colitis & $555-558$ & 0 & 0 & 3 & 6 & 9 \\
\hline -Other Intestinal Dis & $560-569$ & 1 & 0 & 7 & 9 & 17 \\
\hline -Other Digestive Dis & $570-579$ & 0 & 4 & 9 & 14 & 27 \\
\hline GENITOURINARY SYSTEM & $580-629$ & 7 & 14 & 47 & 78 & 146 \\
\hline -Nephritis, Nephrosis & $580-589$ & 0 & 0 & 2 & 3 & 5 \\
\hline -Other Urinary Dis & $590-599$ & 1 & 5 & 22 & 39 & 67 \\
\hline -Male Genital Organ Dis & $600-608$ & 0 & 0 & 0 & 0 & 0 \\
\hline -Breast Disorders & $610-612$ & 0 & 0 & 0 & 8 & 8 \\
\hline -Pelvic Inflammatory Dis & 614-616 & 0 & 5 & 0 & 1 & 6 \\
\hline -Other Female Disorders & 617-629 & 6 & 4 & 23 & 27 & 60 \\
\hline
\end{tabular}

*Only those diagnostic categories and gender/age combinations with at least one occurrence appear in this table. 


\section{Savannah River Site 2010}

\section{Absence Data}

Appendix G. Number of Diagnoses in Each Diagnostic Category by Gender and Age*

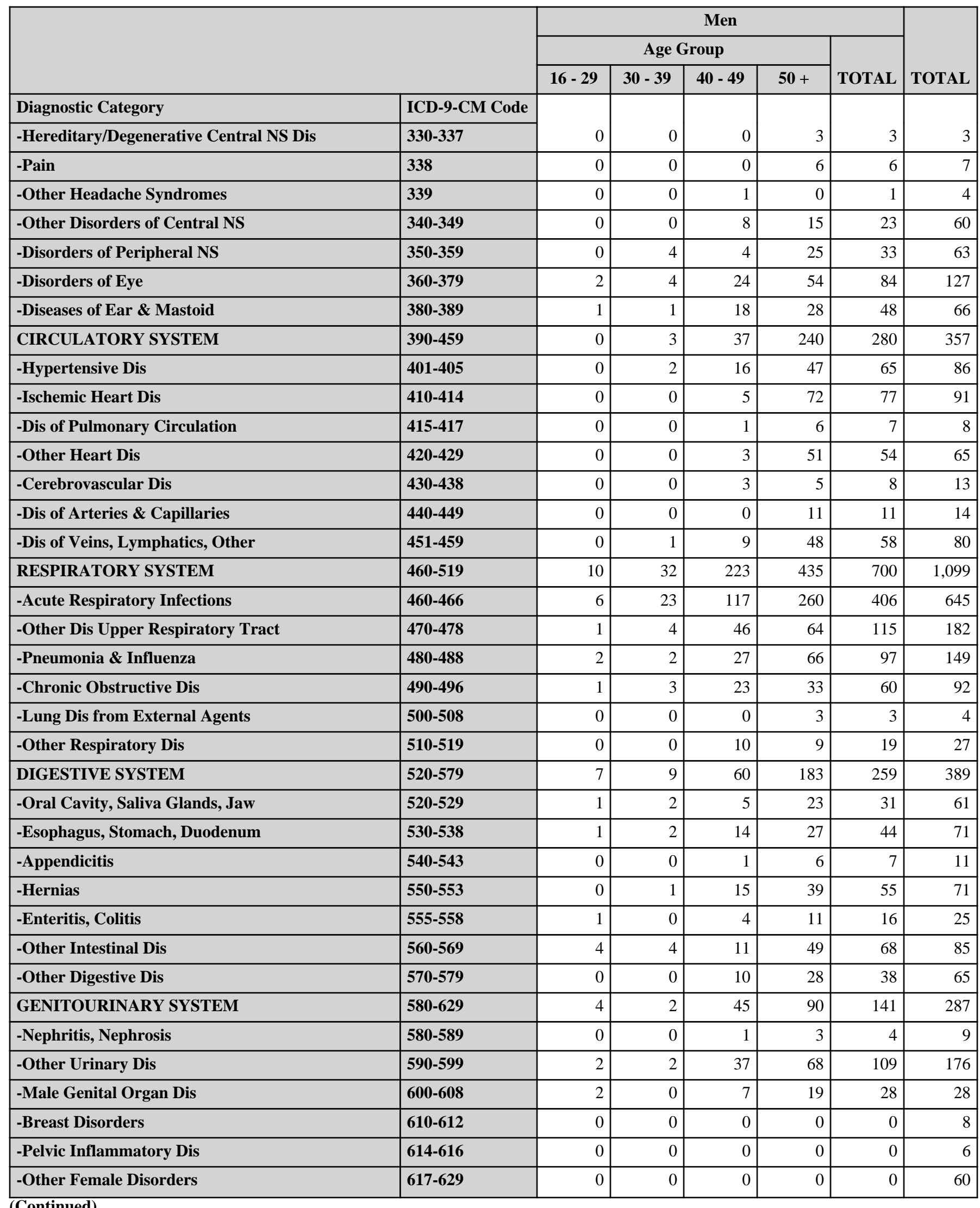

*Only those diagnostic categories and gender/age combinations with at least one occurrence appear in this table. 


\section{Savannah River Site 2010}

\section{Absence Data}

Appendix G. Number of Diagnoses in Each Diagnostic Category by Gender and Age*

\begin{tabular}{|c|c|c|c|c|c|c|}
\hline & & \multicolumn{5}{|c|}{ Women } \\
\hline & & \multicolumn{4}{|c|}{ Age Group } & \multirow[b]{2}{*}{ TOTAL } \\
\hline & & $16-29$ & $30-39$ & $40-49$ & $50+$ & \\
\hline Diagnostic Category & ICD-9-CM Code & \multirow[b]{2}{*}{0} & \multirow[b]{2}{*}{0} & \multirow[b]{2}{*}{11} & \multirow[b]{2}{*}{17} & \multirow[b]{2}{*}{28} \\
\hline SKIN AND SUBCUTANEOUS TISSUE & $680-709$ & & & & & \\
\hline -Infections & $680-686$ & 0 & 0 & 7 & 3 & 10 \\
\hline -Other Inflammatory Conditions & 690-698 & 0 & 0 & 0 & 2 & 2 \\
\hline -Other & 700-709 & 0 & 0 & 4 & 12 & 16 \\
\hline $\begin{array}{l}\text { MUSCULOSKELETAL \& CONNECTIVE } \\
\text { TISSUE }\end{array}$ & 710-739 & 3 & 38 & 77 & 242 & 360 \\
\hline -Arthropathies & $710-719$ & 1 & 6 & 16 & 61 & 84 \\
\hline -Dorsopathies & $720-724$ & 1 & 30 & 35 & 126 & 192 \\
\hline -Rheumatism, Excluding Back & 725-729 & 0 & 2 & 22 & 46 & 70 \\
\hline -Other Dis \& Acquired Deformities & 730-739 & 1 & 0 & 4 & 9 & 14 \\
\hline CONGENITAL ANOMALIES & $740-759$ & 0 & 0 & 1 & 2 & 3 \\
\hline $\begin{array}{l}\text { SYMPTOMS, SIGNS, \& ILL-DEFINED } \\
\text { CONDITIONS }\end{array}$ & $780-799$ & 21 & 29 & 128 & 249 & 427 \\
\hline -Symptoms & 780-789 & 19 & 28 & 125 & 242 & 414 \\
\hline -Non-Specific Abnormal Findings & $790-796$ & 2 & 1 & 3 & 7 & 13 \\
\hline INJURY \& POISONING & $800-999$ & 3 & 16 & 44 & 81 & 144 \\
\hline -Fracture - Skull & $800-804$ & 0 & 0 & 0 & 1 & 1 \\
\hline -Fracture - Neck, Trunk & 805-809 & 0 & 0 & 0 & 2 & 2 \\
\hline -Fracture - Upper Limb & 810-819 & 0 & 2 & 0 & 1 & 3 \\
\hline -Fracture - Lower Limb & $820-829$ & 0 & 1 & 0 & 8 & 9 \\
\hline -Dislocation & 830-839 & 0 & 2 & 2 & 3 & 7 \\
\hline -Sprains \& Strains - Back & 846-847 & 0 & 1 & 4 & 14 & 19 \\
\hline -Sprains \& Strains - Other & $840-845,848$ & 1 & 1 & 7 & 18 & 27 \\
\hline -Intracranial Injury & $850-854$ & 0 & 0 & 1 & 0 & 1 \\
\hline -Internal Injury - Thorax, Abdomen, Pelvis & $860-869$ & 0 & 0 & 0 & 0 & 0 \\
\hline -Open Wound - Head, Neck, Trunk & $870-879$ & 0 & 0 & 0 & 1 & 1 \\
\hline -Open Wound - Upper Limb & 880-887 & 0 & 0 & 1 & 0 & 1 \\
\hline -Open Wound - Lower Limb & $890-897$ & 0 & 0 & 1 & 3 & 4 \\
\hline -Late Effects of Accident & 905-909 & 0 & 0 & 0 & 0 & 0 \\
\hline -Superficial Injury & $910-919$ & 0 & 4 & 2 & 2 & 8 \\
\hline -Contusion & $920-924$ & 1 & 1 & 3 & 6 & 11 \\
\hline -Foreign Body Entering Orifice & 930-939 & 0 & 0 & 0 & 0 & 0 \\
\hline -Burns & $940-949$ & 0 & 0 & 6 & 0 & 6 \\
\hline -Injury to Nerves \& Spinal Cord & 950-957 & 0 & 0 & 0 & 1 & 1 \\
\hline -Complications \& Unspecified Injuries & 958-959 & 0 & 0 & 2 & 2 & 4 \\
\hline -Poisoning - Medicinal/Biological & $960-979$ & 0 & 0 & 2 & 0 & 2 \\
\hline -Toxic Effects - Non-medicinal & $980-989$ & 0 & 0 & 0 & 0 & 0 \\
\hline
\end{tabular}

(Continued)

*Only those diagnostic categories and gender/age combinations with at least one occurrence appear in this table. 


\section{Savannah River Site 2010}

\section{Absence Data}

Appendix G. Number of Diagnoses in Each Diagnostic Category by Gender and Age*

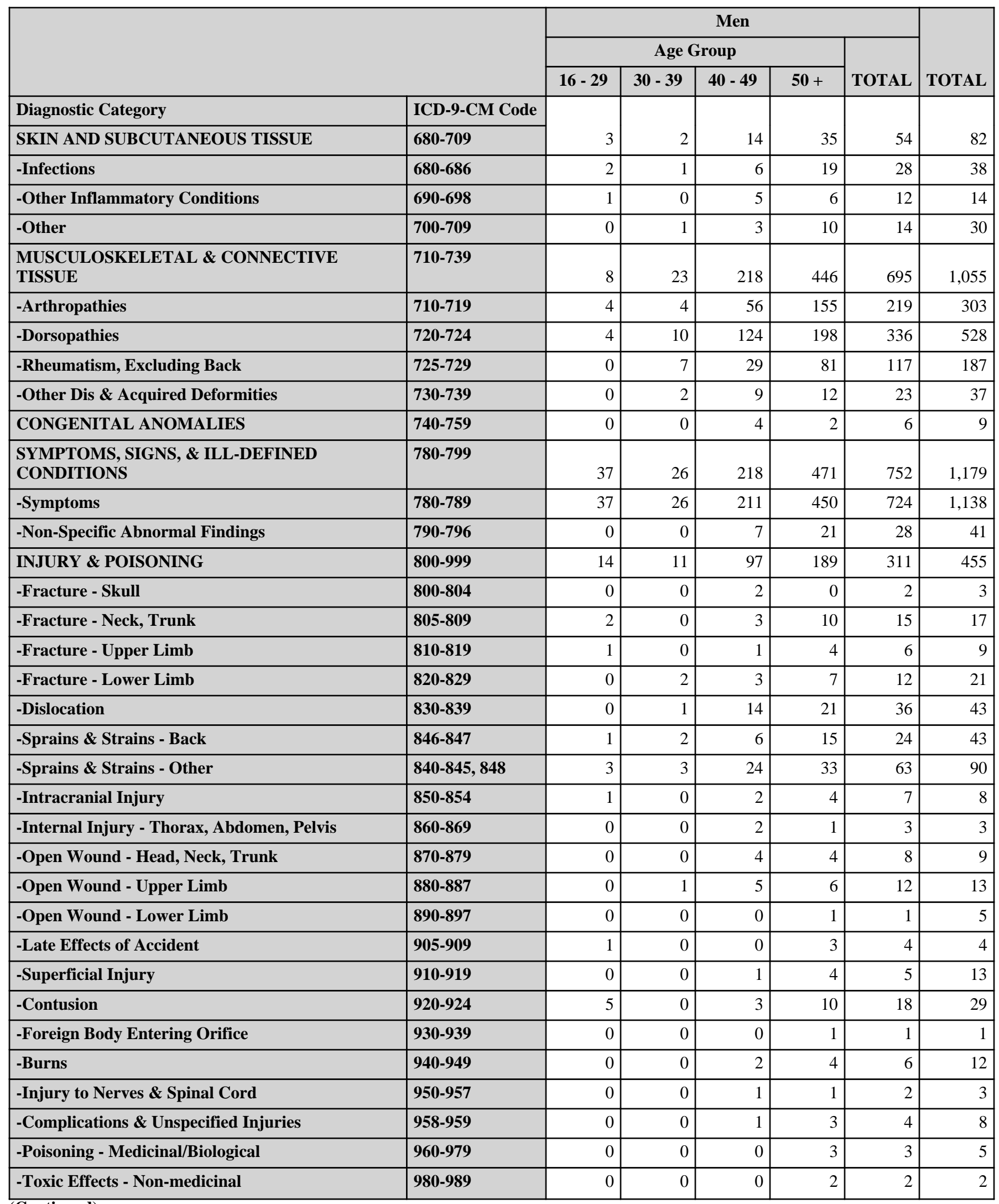

(Continued)

*Only those diagnostic categories and gender/age combinations with at least one occurrence appear in this table. 
Savannah River Site 2010

Absence Data

Appendix G. Number of Diagnoses in Each Diagnostic Category by Gender and Age*

\begin{tabular}{|c|c|c|c|c|c|c|}
\hline & & \multicolumn{5}{|c|}{ Women } \\
\hline & & \multicolumn{4}{|c|}{ Age Group } & \multirow[b]{2}{*}{ TOTAL } \\
\hline & & $16-29$ & $30-39$ & $40-49$ & $\mathbf{5 0 +}$ & \\
\hline Diagnostic Category & ICD-9-CM Code & & & & & \\
\hline -Unspecified Effects - External Causes & $990-995$ & 1 & 0 & 4 & 2 & 7 \\
\hline -Complications of Surgical/Medical Care & 996-999 & 0 & 4 & 9 & 17 & 30 \\
\hline $\begin{array}{l}\text { HEALTH STATUS/HEALTH SERVICE } \\
\text { CONTACT }\end{array}$ & V01-V89 & 4 & 5 & 23 & 39 & 71 \\
\hline -Communicable Dis & V01-V06 & 1 & 0 & 0 & 1 & 2 \\
\hline -Personal \& Family History & V10-V19 & 0 & 0 & 7 & 6 & 13 \\
\hline -Health Services Reproduction/Development & V20-V29 & 2 & 5 & 3 & 0 & 10 \\
\hline -Health Status & V40-V49 & 0 & 0 & 2 & 2 & 4 \\
\hline -Specific Procedures/Aftercare & V50-V59 & 0 & 0 & 9 & 26 & 35 \\
\hline -Other Circumstances & V60-V69 & 1 & 0 & 1 & 0 & 2 \\
\hline -Examination \& Investigation & V70-V82 & 0 & 0 & 0 & 4 & 4 \\
\hline -Other Specified Exposures & V87 & 0 & 0 & 1 & 0 & 1 \\
\hline -Retained Foreign Body & V90 & 0 & 0 & 0 & 0 & 0 \\
\hline
\end{tabular}

*Only those diagnostic categories and gender/age combinations with at least one occurrence appear in this table. 


\section{Savannah River Site 2010}

Absence Data

Appendix G. Number of Diagnoses in Each Diagnostic Category by Gender and Age*

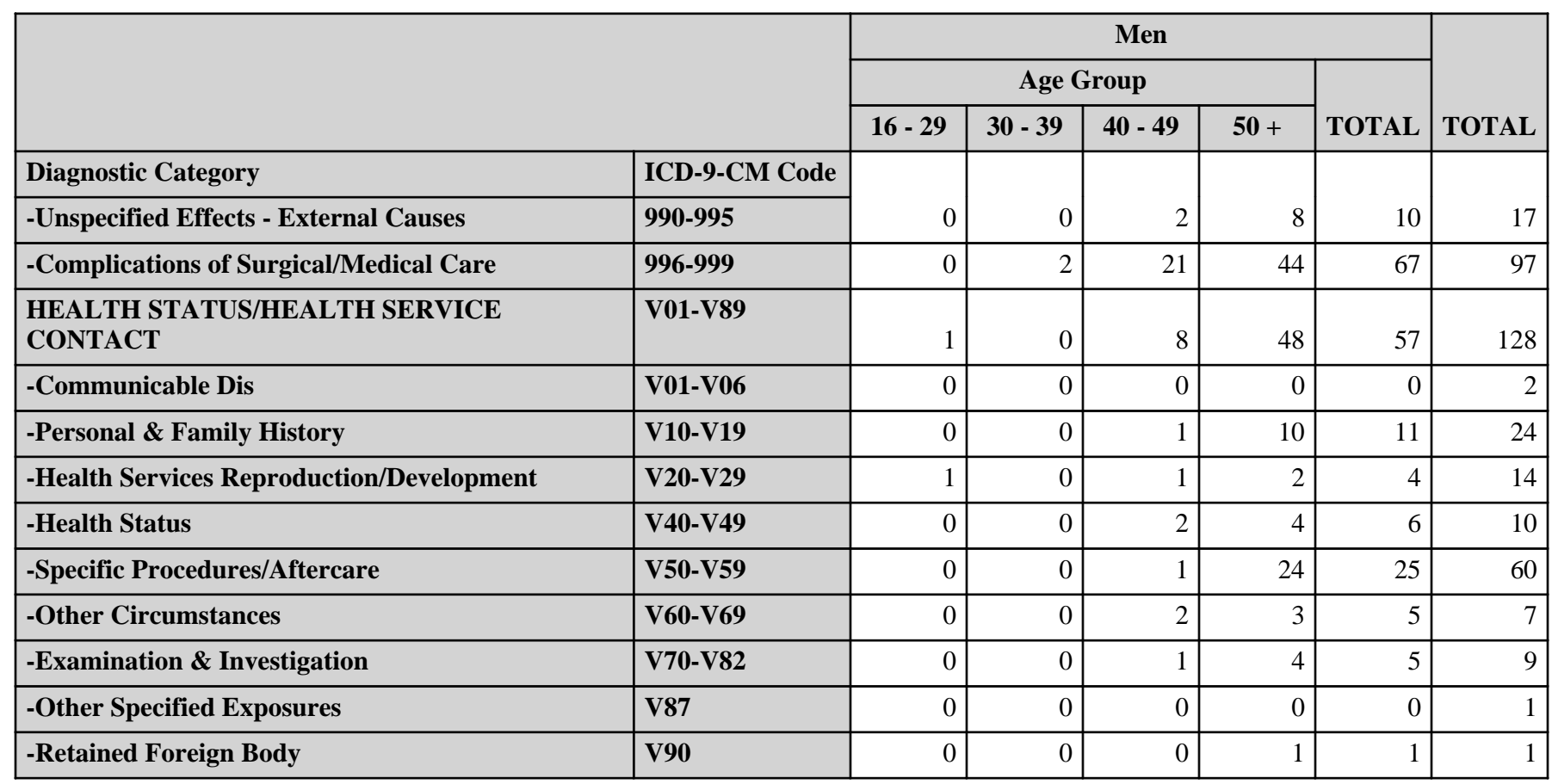

\begin{tabular}{|c|c|c|c|c|c|c|c|c|c|c|c|}
\hline & \multicolumn{5}{|c|}{ Women } & \multicolumn{5}{|c|}{ Men } & \multirow[b]{3}{*}{ TOTAL } \\
\hline & \multicolumn{4}{|c|}{ Age Group } & \multirow[b]{2}{*}{ TOTAL } & \multicolumn{4}{|c|}{ Age Group } & \multirow[b]{2}{*}{ TOTAL } & \\
\hline & $16-29$ & 30 - 39 & $40-49$ & $50+$ & & $16-29$ & 30 - 39 & $40-49$ & $50+$ & & \\
\hline Diagnostic Category & \multirow[b]{2}{*}{73} & \multirow[b]{2}{*}{160} & \multirow[b]{2}{*}{629} & \multirow[b]{2}{*}{1,329} & \multirow[b]{2}{*}{2,191} & \multirow[b]{2}{*}{108} & \multirow[b]{2}{*}{133} & \multirow[b]{2}{*}{1,141} & \multirow[b]{2}{*}{2,565} & \multirow[b]{2}{*}{3,947} & \multirow[b]{2}{*}{6,138} \\
\hline Total & & & & & & & & & & & \\
\hline
\end{tabular}

*Only those diagnostic categories and gender/age combinations with at least one occurrence appear in this table. 
Savannah River Site 2010

Absence Data

Appendix H. Total Number of Calendar Days Absent in Each Diagnostic Category by Gender and Age*

\begin{tabular}{|c|c|c|c|c|c|c|}
\hline & & \multicolumn{5}{|c|}{ Women } \\
\hline & & \multicolumn{4}{|c|}{ Age Group } & \multirow[b]{2}{*}{ TOTAL } \\
\hline & & $16-29$ & $30-39$ & $40-49$ & $50+$ & \\
\hline Diagnostic Category & ICD-9-CM Code & \multirow[b]{2}{*}{20} & \multirow[b]{2}{*}{19} & \multirow[b]{2}{*}{131} & \multirow[b]{2}{*}{626} & \multirow[b]{2}{*}{796} \\
\hline INFECTIOUS \& PARASITIC DISEASES (DIS) & 001-139 & & & & & \\
\hline MALIGNANT NEOPLASMS & $\begin{array}{l}140-208, \\
209.0-209.3, \\
230-234\end{array}$ & 0 & 0 & 394 & 822 & 1,216 \\
\hline BENIGN \& UNCERTAIN NEOPLASMS & $\begin{array}{l}209.4-209.6 \\
210-229,235-239\end{array}$ & 87 & 130 & 285 & 468 & 970 \\
\hline ENDOCRINE/METABOLIC/IMMUNITY & $240-279$ & 0 & 12 & 223 & 454 & 689 \\
\hline BLOOD \& BLOOD-FORMING ORGANS & $280-289$ & 0 & 64 & 16 & 163 & 243 \\
\hline MENTAL DISORDERS & 290-319 & 19 & 0 & 232 & 236 & 487 \\
\hline NERVOUS SYSTEM (NS) \& SENSE ORGANS & $320-389$ & 8 & 132 & 249 & 1,122 & 1,511 \\
\hline CIRCULATORY SYSTEM & $390-459$ & 1 & 9 & 308 & 1,048 & 1,366 \\
\hline RESPIRATORY SYSTEM & $460-519$ & 50 & 196 & 768 & 1,966 & 2,980 \\
\hline DIGESTIVE SYSTEM & $520-579$ & 43 & 68 & 484 & 975 & 1,570 \\
\hline GENITOURINARY SYSTEM & $580-629$ & 107 & 90 & 661 & 1,064 & 1,922 \\
\hline SKIN AND SUBCUTANEOUS TISSUE & 680-709 & 0 & 0 & 134 & 283 & 417 \\
\hline $\begin{array}{l}\text { MUSCULOSKELETAL \& CONNECTIVE } \\
\text { TISSUE }\end{array}$ & 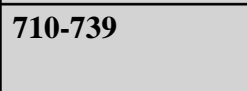 & 57 & 193 & 1,088 & 3,855 & 5,193 \\
\hline CONGENITAL ANOMALIES & $740-759$ & 0 & 0 & 14 & 193 & 207 \\
\hline $\begin{array}{l}\text { SYMPTOMS, SIGNS, \& ILL-DEFINED } \\
\text { CONDITIONS }\end{array}$ & $780-799$ & 74 & 273 & 968 & 2,182 & 3,497 \\
\hline INJURY \& POISONING & $800-999$ & 14 & 170 & 794 & 1,642 & 2,620 \\
\hline
\end{tabular}

\footnotetext{
*Absences with >1 ICD-9-CM code in the same diagnostic category were counted only once. Only those diagnostic categories and gender/age combinations with at least one occurrence appear in this table.
} 
Savannah River Site 2010

Absence Data

Appendix H. Total Number of Calendar Days Absent in Each Diagnostic Category by Gender and Age*

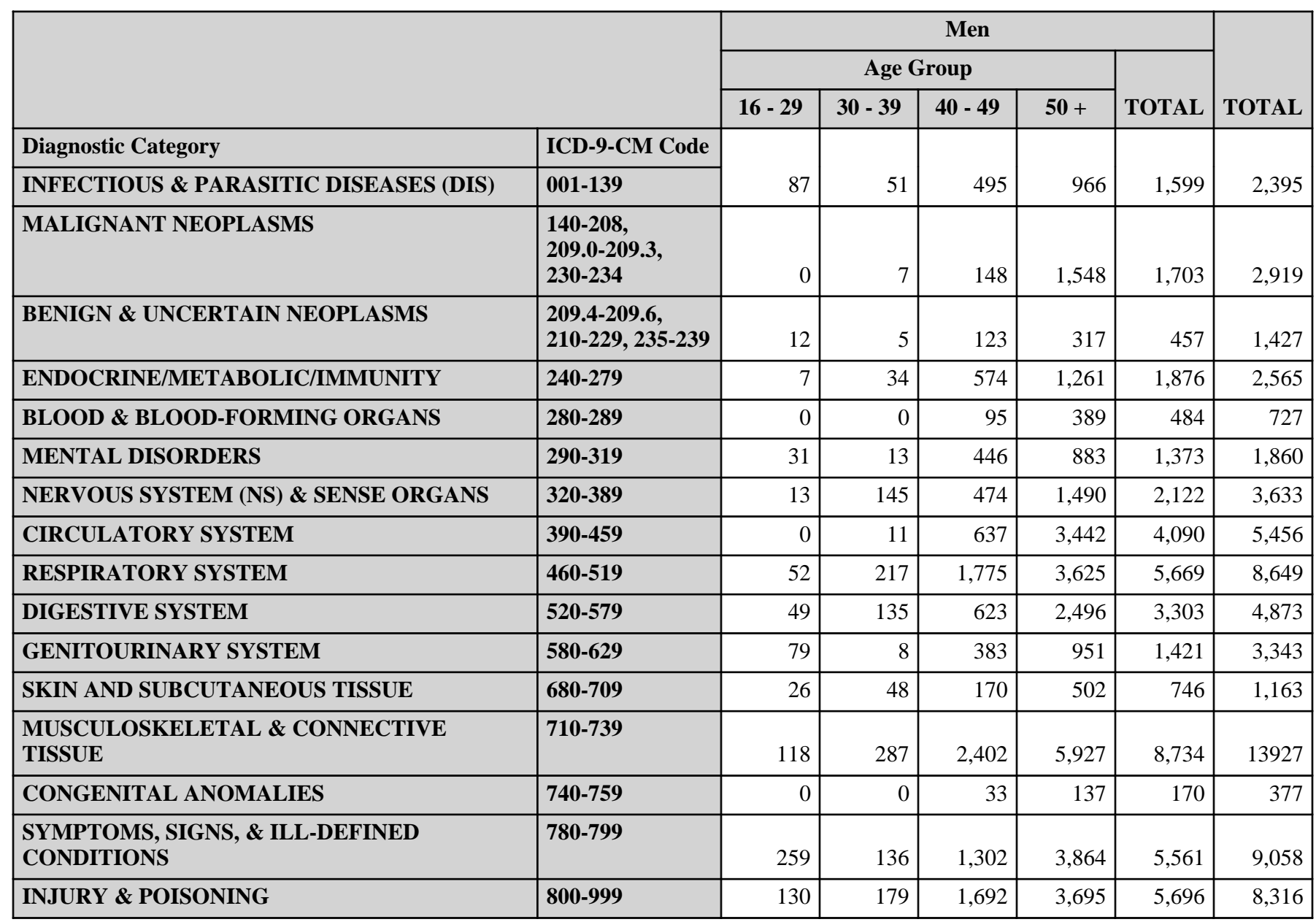

\footnotetext{
*Absences with >1 ICD-9-CM code in the same diagnostic category were counted only once. Only those diagnostic categories and gender/age combinations with at least one occurrence appear in this table.
} 


\section{Savannah River Site 2010}

\section{Absence Data}

Appendix I. Number of Diagnoses in Each Diagnostic Category by Gender and Job Category*

\begin{tabular}{|c|c|c|c|c|}
\hline & \multicolumn{3}{|c|}{ Women } \\
\hline & & \multicolumn{3}{|c|}{ Job Category } \\
\hline & & Professional & $\begin{array}{c}\text { Administrative } \\
\text { Support }\end{array}$ & Technical Support \\
\hline Diagnostic Category & ICD-9-CM Code & \multirow[b]{2}{*}{14} & \multirow[b]{2}{*}{13} & \multirow[b]{2}{*}{29} \\
\hline INFECTIOUS \& PARASITIC DISEASES (DIS) & 001-139 & & & \\
\hline -Intestinal Infectious Dis & \begin{tabular}{|c|}
$001-009$ \\
\end{tabular} & 6 & 5 & 13 \\
\hline -Other Bacterial Dis & 030-041 & 1 & 5 & 6 \\
\hline -Viral Dis with Exanthem & $050-059$ & 2 & 1 & 1 \\
\hline -Other Viral Dis \& Chlamydiae & 070-079 & 5 & 2 & 6 \\
\hline -Syphilis \& Other Venereal Dis & 090-099 & 0 & 0 & 1 \\
\hline -Mycoses & $110-118$ & 0 & 0 & 2 \\
\hline MALIGNANT NEOPLASMS & $\begin{array}{l}140-208, \\
209.0-209.3 \\
230-234\end{array}$ & 14 & 28 & 7 \\
\hline -Lip, Oral Cavity, Pharynx & 140-149 & 0 & 1 & 0 \\
\hline -Digestive \& Peritoneal & $150-159$ & 0 & 0 & 0 \\
\hline -Respiratory \& Intrathoracic & $160-165$ & 0 & 5 & 1 \\
\hline -Bone, Connective Tissue, Skin & $170-173,176$ & 0 & 1 & 1 \\
\hline -Breast & $174-175$ & 14 & 7 & 5 \\
\hline -Genitourinary & 179-189 & 0 & 6 & 0 \\
\hline -Endocrine & $\begin{array}{l}\text { 193-194, } \\
\text { 209.0-209.3 }\end{array}$ & 0 & 2 & 0 \\
\hline -Other \& Unspecified Sites & 195-199, 209.7 & 0 & 6 & 0 \\
\hline -Lymphatic \& Hematopoietic & $200-208$ & 0 & 0 & 0 \\
\hline BENIGN \& UNCERTAIN NEOPLASMS & $\begin{array}{l}\text { 209.4-209.6, } \\
210-229,235-239\end{array}$ & 15 & 7 & 13 \\
\hline ENDOCRINE/METABOLIC/IMMUNITY & $240-279$ & 7 & 20 & 11 \\
\hline -Thyroid Gland Disorders & $240-246$ & 2 & 1 & 0 \\
\hline -Other Endocrine Gland Dis & 249-259 & 2 & 12 & 3 \\
\hline -Other Metabolic \& Immunity Disorders & $270-279$ & 3 & 7 & 8 \\
\hline BLOOD \& BLOOD-FORMING ORGANS & $280-289$ & 2 & 9 & 5 \\
\hline MENTAL DISORDERS & $290-319$ & 3 & 8 & 4 \\
\hline -Psychoses & 290-299 & 2 & 2 & 0 \\
\hline -Non-Psychotic Disorders & $300-302,306-316$ & 1 & 3 & 4 \\
\hline -Drug Dependence & 304-305 & 0 & 3 & 0 \\
\hline NERVOUS SYSTEM (NS) \& SENSE ORGANS & $320-389$ & 17 & 40 & 46 \\
\hline -Pain & 338 & 0 & 0 & 1 \\
\hline -Other Headache Syndromes & 339 & 0 & 1 & 1 \\
\hline -Other Disorders of Central NS & \begin{tabular}{|l|}
$340-349$ \\
\end{tabular} & 2 & 6 & 22 \\
\hline -Disorders of Peripheral NS & $350-359$ & 6 & 10 & 8 \\
\hline -Disorders of Eye & $360-379$ & 8 & 20 & 7 \\
\hline
\end{tabular}

(Continued)

*Only those diagnostic categories and gender/job category combinations with at least one occurrence appear in this table. 


\section{Savannah River Site 2010}

\section{Absence Data}

Appendix I. Number of Diagnoses in Each Diagnostic Category by Gender and Job Category*

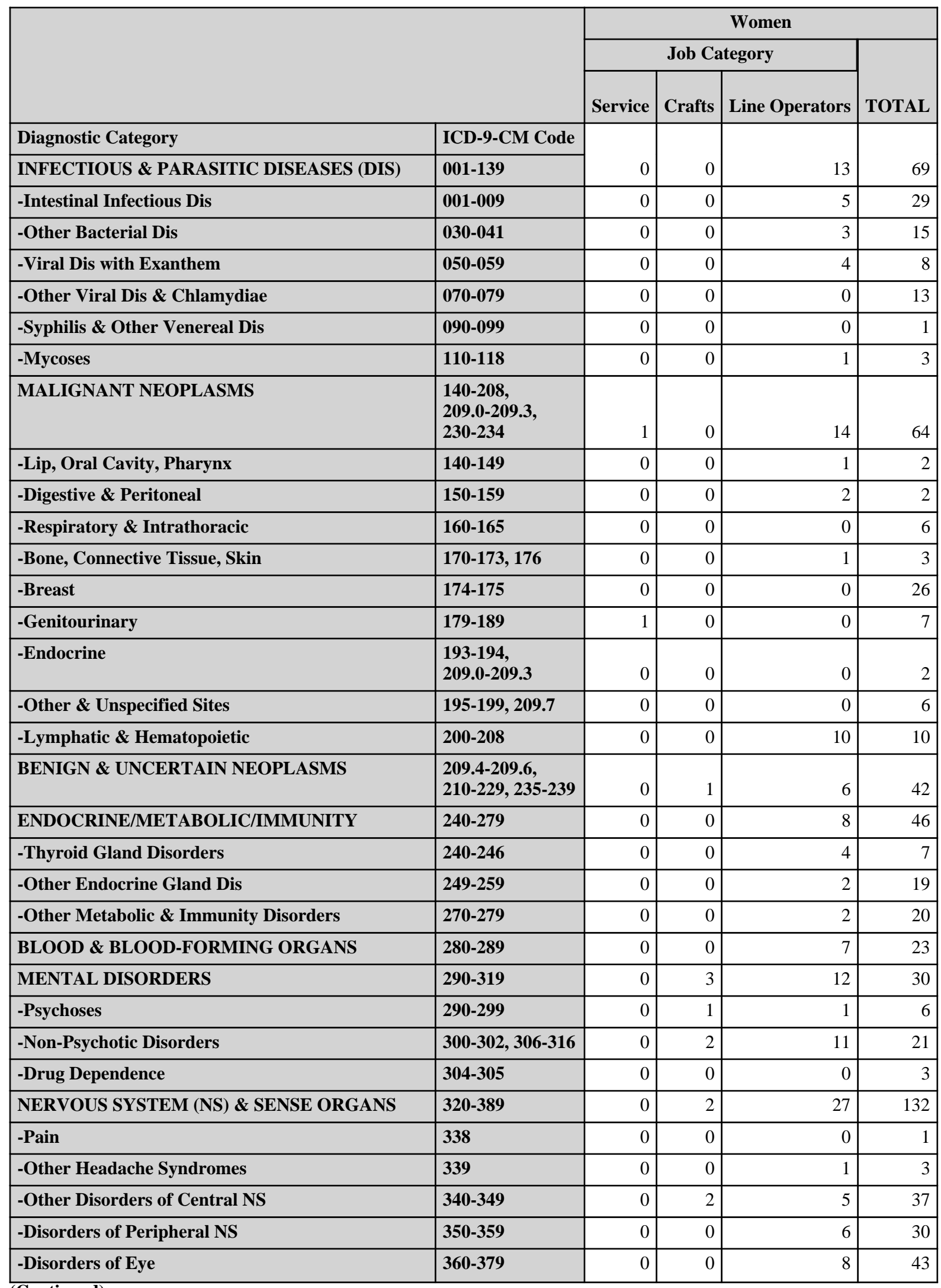

(Continued)

*Only those diagnostic categories and gender/job category combinations with at least one occurrence appear in this table. 


\section{Savannah River Site 2010}

\section{Absence Data}

Appendix I. Number of Diagnoses in Each Diagnostic Category by Gender and Job Category*

\begin{tabular}{|c|c|c|c|c|}
\hline & \multicolumn{3}{|c|}{ Women } \\
\hline & & \multicolumn{3}{|c|}{ Job Category } \\
\hline & & Professional & $\begin{array}{c}\text { Administrative } \\
\text { Support }\end{array}$ & Technical Support \\
\hline Diagnostic Category & ICD-9-CM Code & \multirow[b]{2}{*}{1} & \multirow[b]{2}{*}{3} & \multirow[b]{2}{*}{7} \\
\hline -Diseases of Ear \& Mastoid & $380-389$ & & & \\
\hline CIRCULATORY SYSTEM & $390-459$ & 19 & 9 & 19 \\
\hline -Hypertensive Dis & 401-405 & 3 & 5 & 1 \\
\hline -Ischemic Heart Dis & $410-414$ & 3 & 3 & 2 \\
\hline -Dis of Pulmonary Circulation & $415-417$ & 0 & 0 & 1 \\
\hline -Other Heart Dis & $420-429$ & 6 & 0 & 3 \\
\hline -Cerebrovascular Dis & $430-438$ & 4 & 0 & 0 \\
\hline -Dis of Arteries \& Capillaries & $440-449$ & 0 & 0 & 2 \\
\hline -Dis of Veins, Lymphatics, Other & 451-459 & 3 & 1 & 10 \\
\hline RESPIRATORY SYSTEM & $460-519$ & 82 & 83 & 133 \\
\hline -Acute Respiratory Infections & $460-466$ & 47 & 46 & 84 \\
\hline -Other Dis Upper Respiratory Tract & $470-478$ & 12 & 15 & 21 \\
\hline -Pneumonia \& Influenza & $480-488$ & 15 & 12 & 15 \\
\hline -Chronic Obstructive Dis & $490-496$ & 6 & 9 & 10 \\
\hline -Lung Dis from External Agents & $500-508$ & 0 & 0 & 1 \\
\hline -Other Respiratory Dis & $510-519$ & 2 & 1 & 2 \\
\hline DIGESTIVE SYSTEM & $520-579$ & 21 & 29 & 46 \\
\hline -Oral Cavity, Saliva Glands, Jaw & $520-529$ & 1 & 11 & 12 \\
\hline -Esophagus, Stomach, Duodenum & $530-538$ & 7 & 5 & 9 \\
\hline -Appendicitis & \begin{tabular}{|l|l|}
$540-543$ \\
\end{tabular} & 1 & 0 & 2 \\
\hline -Hernias & $550-553$ & 4 & 4 & 5 \\
\hline -Enteritis, Colitis & $555-558$ & 2 & 0 & 7 \\
\hline -Other Intestinal Dis & $560-569$ & 4 & 2 & 7 \\
\hline -Other Digestive Dis & $570-579$ & 2 & 7 & 4 \\
\hline GENITOURINARY SYSTEM & $580-629$ & 28 & 33 & 54 \\
\hline -Nephritis, Nephrosis & $580-589$ & 1 & 1 & 0 \\
\hline -Other Urinary Dis & $590-599$ & 12 & 15 & 21 \\
\hline -Breast Disorders & $610-612$ & 1 & 2 & 5 \\
\hline -Pelvic Inflammatory Dis & 614-616 & 0 & 1 & 5 \\
\hline -Other Female Disorders & $617-629$ & 14 & 14 & 23 \\
\hline SKIN AND SUBCUTANEOUS TISSUE & $680-709$ & 5 & 1 & 14 \\
\hline -Infections & $680-686$ & 3 & 0 & 4 \\
\hline -Other Inflammatory Conditions & 690-698 & 0 & 0 & 2 \\
\hline -Other & 700-709 & 2 & 1 & 8 \\
\hline $\begin{array}{l}\text { MUSCULOSKELETAL \& CONNECTIVE } \\
\text { TISSUE }\end{array}$ & $710-739$ & 56 & 61 & 149 \\
\hline
\end{tabular}

(Continued)

*Only those diagnostic categories and gender/job category combinations with at least one occurrence appear in this table. 


\section{Savannah River Site 2010}

\section{Absence Data}

Appendix I. Number of Diagnoses in Each Diagnostic Category by Gender and Job Category*

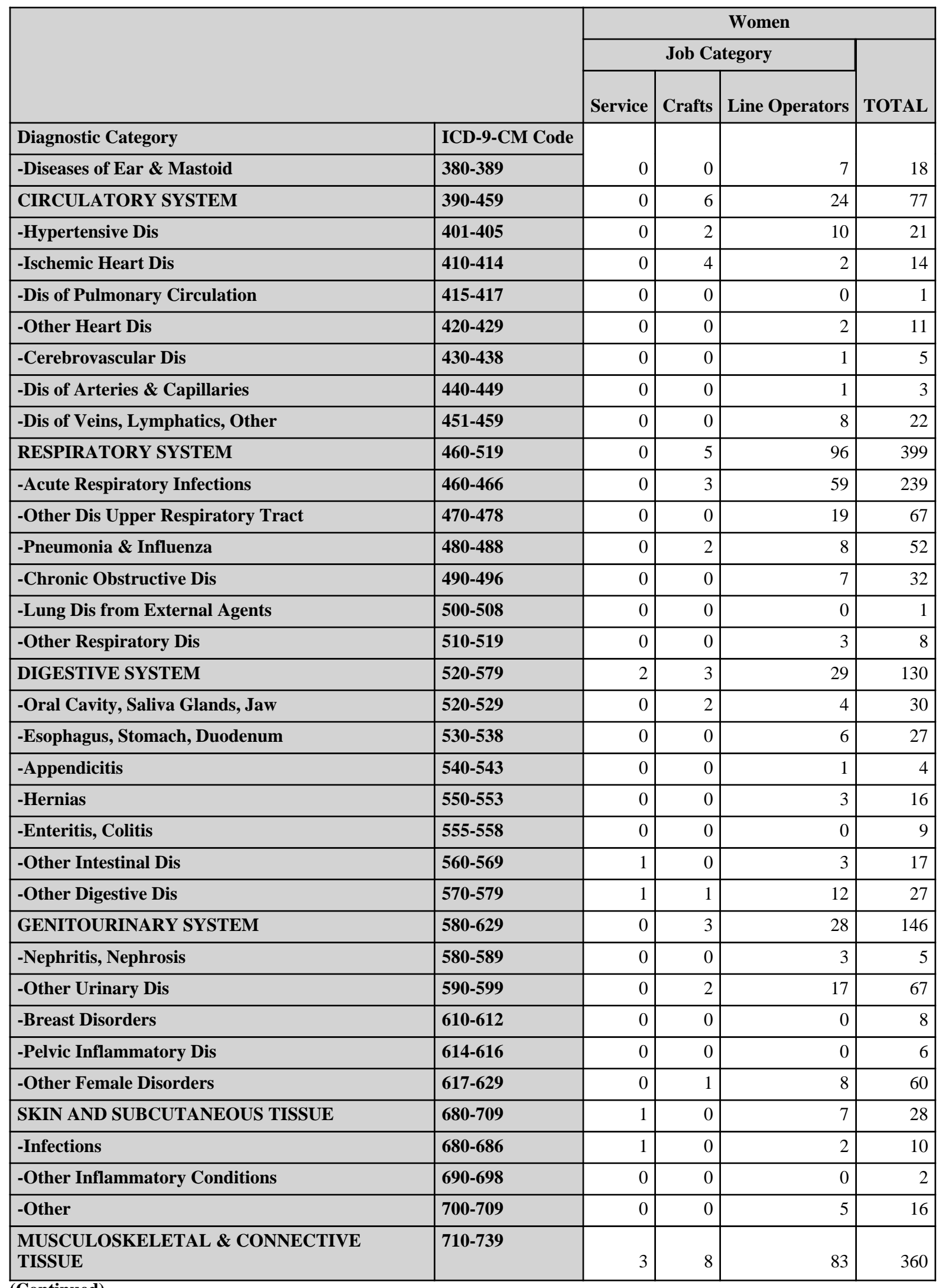

(Continued)

*Only those diagnostic categories and gender/job category combinations with at least one occurrence appear in this table. 


\section{Savannah River Site 2010}

\section{Absence Data}

Appendix I. Number of Diagnoses in Each Diagnostic Category by Gender and Job Category*

\begin{tabular}{|c|c|c|c|c|}
\hline & \multicolumn{3}{|c|}{ Women } \\
\hline & & \multicolumn{3}{|c|}{ Job Category } \\
\hline & & Professional & $\begin{array}{l}\text { Administrative } \\
\text { Support }\end{array}$ & Technical Support \\
\hline Diagnostic Category & ICD-9-CM Code & \multirow[b]{2}{*}{16} & \multirow[b]{2}{*}{16} & \multirow[b]{2}{*}{25} \\
\hline -Arthropathies & \begin{tabular}{|l|}
$710-719$ \\
\end{tabular} & & & \\
\hline -Dorsopathies & $720-724$ & 25 & 30 & 90 \\
\hline -Rheumatism, Excluding Back & $725-729$ & 11 & 11 & 31 \\
\hline -Other Dis \& Acquired Deformities & \begin{tabular}{|l|}
$730-739$ \\
\end{tabular} & 4 & 4 & 3 \\
\hline CONGENITAL ANOMALIES & $740-759$ & 0 & 1 & 0 \\
\hline $\begin{array}{l}\text { SYMPTOMS, SIGNS, \& ILL-DEFINED } \\
\text { CONDITIONS }\end{array}$ & 780-799 & 70 & 95 & 130 \\
\hline -Symptoms & $780-789$ & 69 & 93 & 126 \\
\hline -Non-Specific Abnormal Findings & $790-796$ & 1 & 2 & 4 \\
\hline INJURY \& POISONING & $800-999$ & 33 & 28 & 47 \\
\hline -Fracture - Skull & $800-804$ & 0 & 1 & 0 \\
\hline -Fracture - Neck, Trunk & \begin{tabular}{|c|}
$805-809$ \\
\end{tabular} & 0 & 0 & 0 \\
\hline -Fracture - Upper Limb & 810-819 & 2 & 1 & 0 \\
\hline -Fracture - Lower Limb & $820-829$ & 1 & 2 & 5 \\
\hline -Dislocation & $830-839$ & 2 & 1 & 3 \\
\hline -Sprains \& Strains - Back & 846-847 & 5 & 3 & 5 \\
\hline -Sprains \& Strains - Other & $840-845,848$ & 5 & 3 & 7 \\
\hline -Intracranial Injury & $850-854$ & 0 & 0 & 0 \\
\hline -Open Wound - Head, Neck, Trunk & $870-879$ & 0 & 1 & 0 \\
\hline -Open Wound - Upper Limb & 880-887 & 0 & 0 & 1 \\
\hline -Open Wound - Lower Limb & 890-897 & 2 & 1 & 1 \\
\hline -Superficial Injury & 910-919 & 1 & 0 & 7 \\
\hline -Contusion & \begin{tabular}{|c|}
$920-924$ \\
\end{tabular} & 1 & 4 & 4 \\
\hline -Burns & $940-949$ & 0 & 0 & 6 \\
\hline -Injury to Nerves \& Spinal Cord & $950-957$ & 1 & 0 & 0 \\
\hline -Complications \& Unspecified Injuries & 958-959 & 1 & 1 & 0 \\
\hline -Poisoning - Medicinal/Biological & $960-979$ & 0 & 2 & 0 \\
\hline -Unspecified Effects - External Causes & $990-995$ & 1 & 1 & 1 \\
\hline -Complications of Surgical/Medical Care & 996-999 & 11 & 7 & 7 \\
\hline $\begin{array}{l}\text { HEALTH STATUS/HEALTH SERVICE } \\
\text { CONTACT }\end{array}$ & V01-V89 & 19 & 25 & 8 \\
\hline -Communicable Dis & V01-V06 & 0 & 1 & 1 \\
\hline -Personal \& Family History & V10-V19 & 3 & 4 & 2 \\
\hline -Health Services Reproduction/Development & V20-V29 & 6 & 0 & 2 \\
\hline -Health Status & V40-V49 & 0 & 1 & 1 \\
\hline -Specific Procedures/Aftercare & V50-V59 & 6 & 19 & 2 \\
\hline -Other Circumstances & V60-V69 & 1 & 0 & 0 \\
\hline
\end{tabular}

(Continued)

*Only those diagnostic categories and gender/job category combinations with at least one occurrence appear in this table. 


\section{Savannah River Site 2010}

\section{Absence Data}

Appendix I. Number of Diagnoses in Each Diagnostic Category by Gender and Job Category*

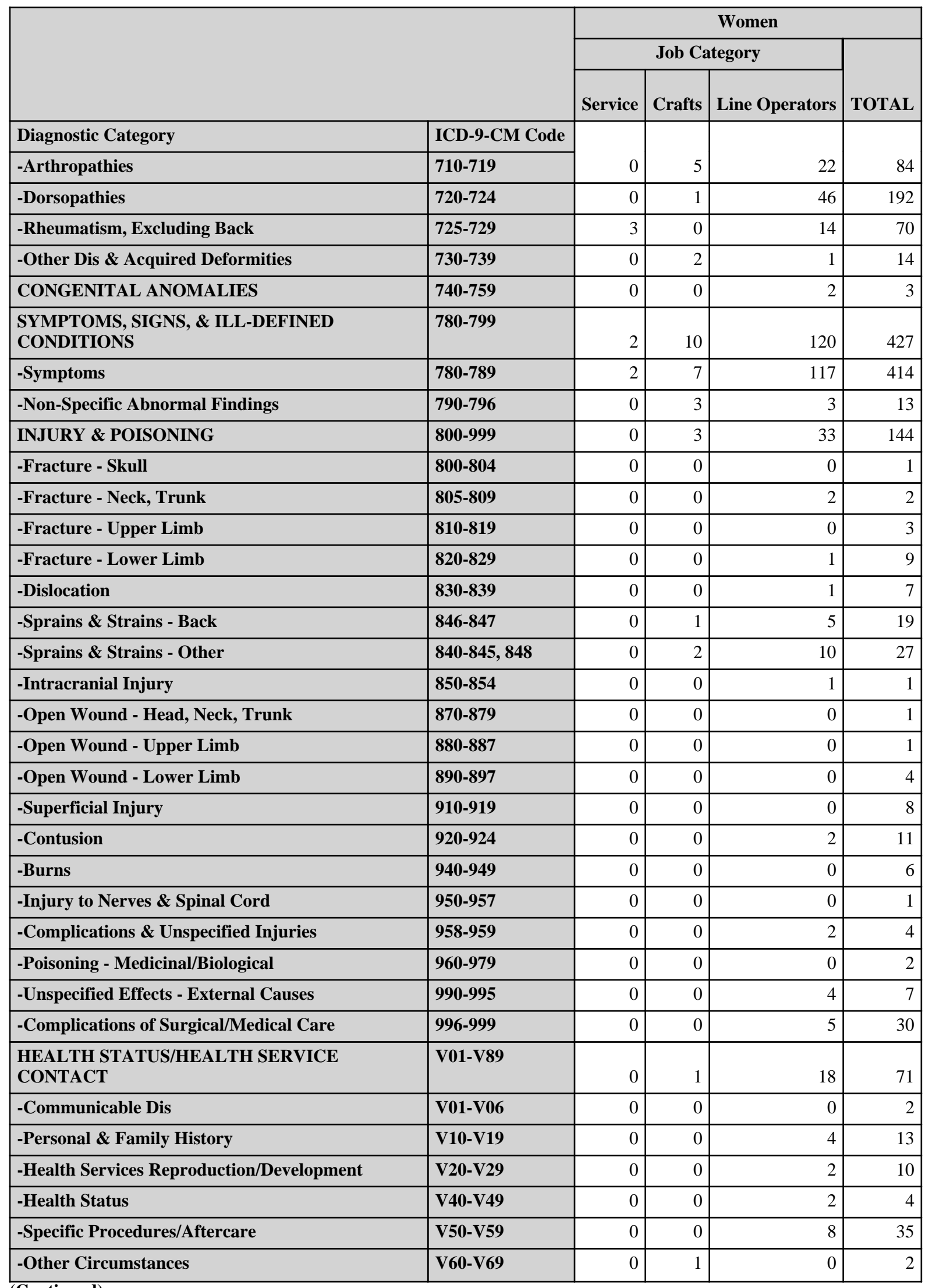

(Continued)

*Only those diagnostic categories and gender/job category combinations with at least one occurrence appear in this table. 


\section{Savannah River Site 2010}

Absence Data

Appendix I. Number of Diagnoses in Each Diagnostic Category by Gender and Job Category*

\begin{tabular}{|c|c|c|c|c|}
\hline & & \multicolumn{3}{|c|}{ Women } \\
\hline & & \multicolumn{3}{|c|}{ Job Category } \\
\hline & & Professional & $\begin{array}{l}\text { Administrative } \\
\text { Support }\end{array}$ & Technical Support \\
\hline Diagnostic Category & ICD-9-CM Code & \multirow[b]{2}{*}{3} & \multirow[b]{2}{*}{0} & \multirow[b]{2}{*}{0} \\
\hline -Examination \& Investigation & V70-V82 & & & \\
\hline -Other Specified Exposures & V87 & 0 & 0 & 0 \\
\hline
\end{tabular}

*Only those diagnostic categories and gender/job category combinations with at least one occurrence appear in this table. 
Savannah River Site 2010

Absence Data

Appendix I. Number of Diagnoses in Each Diagnostic Category by Gender and Job Category*

\begin{tabular}{|c|c|c|c|c|c|}
\hline & & \multicolumn{4}{|c|}{ Women } \\
\hline & & \multicolumn{3}{|c|}{ Job Category } & \multirow[b]{2}{*}{ TOTAL } \\
\hline & & Service & Crafts & Line Operators & \\
\hline Diagnostic Category & ICD-9-CM Code & \multirow[b]{2}{*}{0} & \multirow[b]{2}{*}{0} & \multirow[b]{2}{*}{1} & \multirow[b]{2}{*}{4} \\
\hline -Examination \& Investigation & V70-V82 & & & & \\
\hline -Other Specified Exposures & V87 & 0 & 0 & 1 & 1 \\
\hline
\end{tabular}

\begin{tabular}{|c|c|c|c|c|c|c|c|}
\hline & \multicolumn{7}{|c|}{ Women } \\
\hline & \multicolumn{6}{|c|}{ Job Category } & \multirow[b]{2}{*}{ TOTAL } \\
\hline & Professional & $\begin{array}{c}\text { Administrative } \\
\text { Support }\end{array}$ & Technical Support & Service & Crafts & Line Operators & \\
\hline Diagnostic Category & \multirow[b]{2}{*}{405} & \multirow[b]{2}{*}{490} & \multirow[b]{2}{*}{715} & \multirow[b]{2}{*}{9} & \multirow[b]{2}{*}{45} & \multirow[b]{2}{*}{527} & \multirow[b]{2}{*}{2,191} \\
\hline Total & & & & & & & \\
\hline
\end{tabular}

*Only those diagnostic categories and gender/job category combinations with at least one occurrence appear in this table. 


\section{Savannah River Site 2010}

\section{Absence Data}

Appendix I. Number of Diagnoses in Each Diagnostic Category by Gender and Job Category*

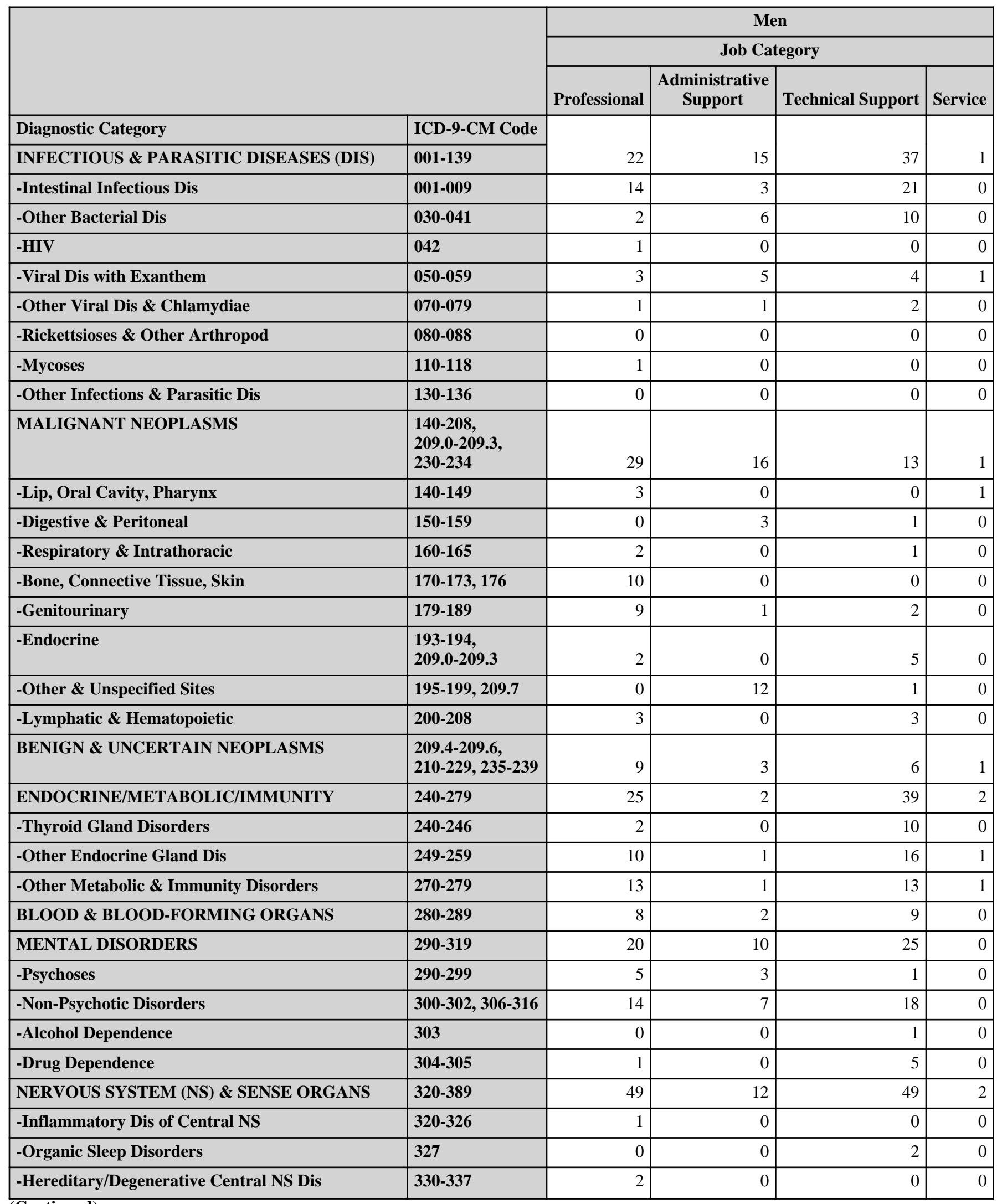

(Continued)

*Only those diagnostic categories and gender/job category combinations with at least one occurrence appear in this table. 


\section{Savannah River Site 2010}

\section{Absence Data}

Appendix I. Number of Diagnoses in Each Diagnostic Category by Gender and Job Category*

\begin{tabular}{|c|c|c|c|c|c|}
\hline & & \multicolumn{4}{|c|}{ Men } \\
\hline & & \multicolumn{3}{|c|}{ Job Category } & \multirow[b]{2}{*}{ TOTAL } \\
\hline & & Security and Fire & Crafts & Line Operators & \\
\hline Diagnostic Category & ICD-9-CM Code & \multirow[b]{2}{*}{2} & \multirow[b]{2}{*}{9} & \multirow[b]{2}{*}{54} & \multirow[b]{2}{*}{140} \\
\hline INFECTIOUS \& PARASITIC DISEASES (DIS) & 001-139 & & & & \\
\hline -Intestinal Infectious Dis & \begin{tabular}{|c|}
$001-009$ \\
\end{tabular} & 1 & 5 & 34 & 78 \\
\hline -Other Bacterial Dis & 030-041 & 1 & 3 & 10 & 32 \\
\hline$-\mathrm{HIV}$ & 042 & 0 & 0 & 0 & 1 \\
\hline -Viral Dis with Exanthem & $050-059$ & 0 & 0 & 4 & 17 \\
\hline -Other Viral Dis \& Chlamydiae & 070-079 & 0 & 1 & 4 & 9 \\
\hline -Rickettsioses \& Other Arthropod & 080-088 & 0 & 0 & 1 & 1 \\
\hline -Mycoses & 110-118 & 0 & 0 & 0 & 1 \\
\hline -Other Infections \& Parasitic Dis & $130-136$ & 0 & 0 & 1 & 1 \\
\hline MALIGNANT NEOPLASMS & $\begin{array}{l}140-208, \\
209.0-209.3 \\
230-234\end{array}$ & 0 & 5 & 12 & 76 \\
\hline -Lip, Oral Cavity, Pharynx & $140-149$ & 0 & 1 & 0 & 5 \\
\hline -Digestive \& Peritoneal & $150-159$ & 0 & 0 & 1 & 5 \\
\hline -Respiratory \& Intrathoracic & $160-165$ & 0 & 0 & 0 & 3 \\
\hline -Bone, Connective Tissue, Skin & $170-173,176$ & 0 & 3 & 5 & 18 \\
\hline -Genitourinary & 179-189 & 0 & 1 & 4 & 17 \\
\hline -Endocrine & $\begin{array}{l}193-194, \\
209.0-209.3\end{array}$ & 0 & 0 & 0 & 7 \\
\hline -Other \& Unspecified Sites & 195-199, 209.7 & 0 & 0 & 1 & 14 \\
\hline -Lymphatic \& Hematopoietic & $200-208$ & 0 & 0 & 1 & 7 \\
\hline BENIGN \& UNCERTAIN NEOPLASMS & $\begin{array}{l}209.4-209.6 \\
210-229,235-239\end{array}$ & 0 & 3 & 7 & 29 \\
\hline ENDOCRINE/METABOLIC/IMMUNITY & $240-279$ & 0 & 10 & 54 & 132 \\
\hline -Thyroid Gland Disorders & $240-246$ & 0 & 0 & 2 & 14 \\
\hline -Other Endocrine Gland Dis & $249-259$ & 0 & 3 & 14 & 45 \\
\hline -Other Metabolic \& Immunity Disorders & $270-279$ & 0 & 7 & 38 & 73 \\
\hline BLOOD \& BLOOD-FORMING ORGANS & $280-289$ & 0 & 0 & 10 & 29 \\
\hline MENTAL DISORDERS & 290-319 & 2 & 14 & 13 & 84 \\
\hline -Psychoses & $290-299$ & 0 & 4 & 1 & 14 \\
\hline -Non-Psychotic Disorders & $300-302,306-316$ & 2 & 9 & 7 & 57 \\
\hline -Alcohol Dependence & 303 & 0 & 0 & 0 & 1 \\
\hline -Drug Dependence & $304-305$ & 0 & 1 & 5 & 12 \\
\hline NERVOUS SYSTEM (NS) \& SENSE ORGANS & 320-389 & 0 & 14 & 76 & 202 \\
\hline -Inflammatory Dis of Central NS & $320-326$ & 0 & 0 & 0 & 1 \\
\hline -Organic Sleep Disorders & 327 & 0 & 0 & 1 & 3 \\
\hline -Hereditary/Degenerative Central NS Dis & 330-337 & 0 & 0 & 1 & 3 \\
\hline
\end{tabular}

(Continued)

*Only those diagnostic categories and gender/job category combinations with at least one occurrence appear in this table. 


\section{Savannah River Site 2010}

\section{Absence Data}

Appendix I. Number of Diagnoses in Each Diagnostic Category by Gender and Job Category*

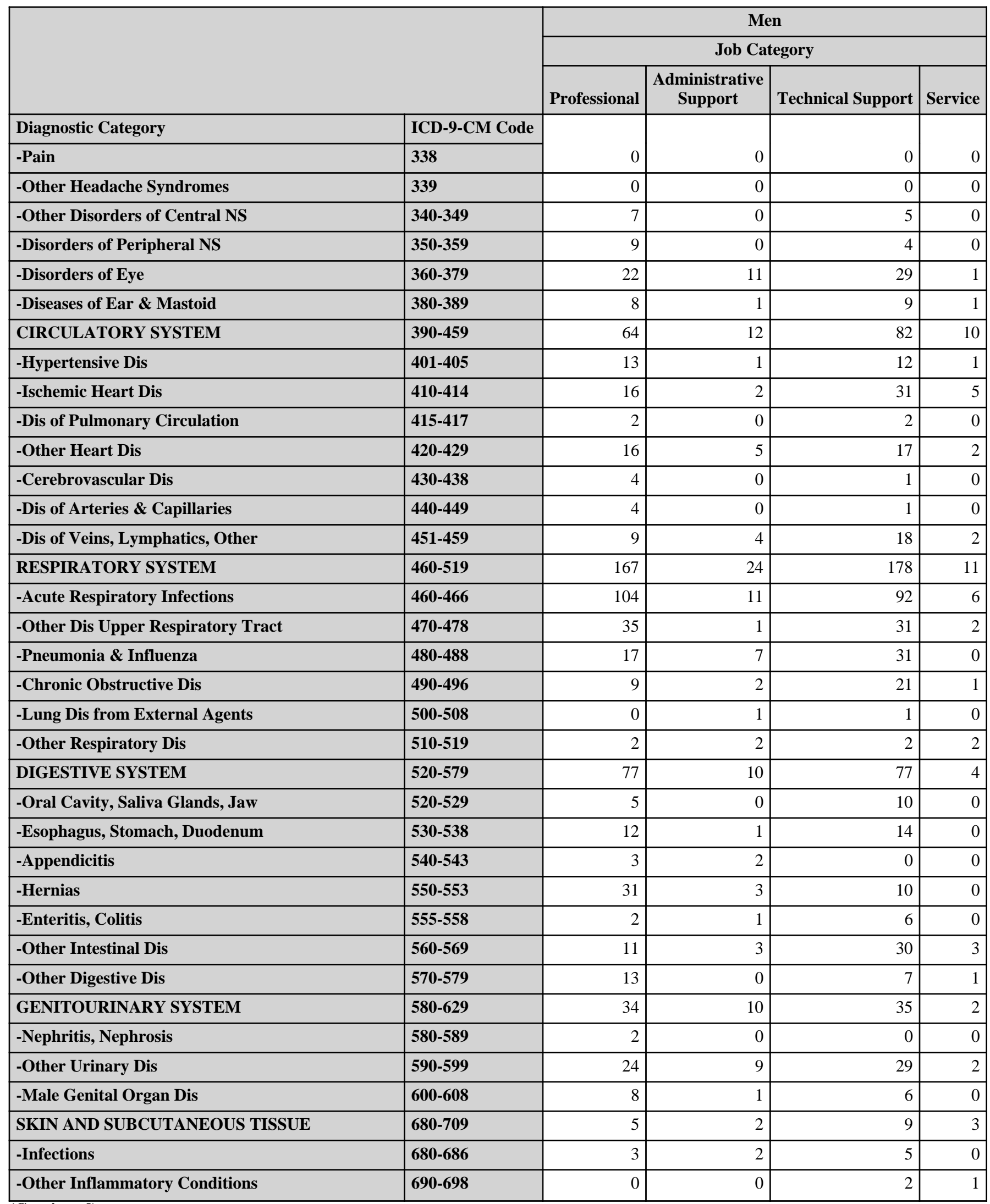

(Continued)

*Only those diagnostic categories and gender/job category combinations with at least one occurrence appear in this table. 


\section{Savannah River Site 2010}

\section{Absence Data}

Appendix I. Number of Diagnoses in Each Diagnostic Category by Gender and Job Category*

\begin{tabular}{|c|c|c|c|c|c|}
\hline & & \multicolumn{4}{|c|}{ Men } \\
\hline & & \multicolumn{3}{|c|}{ Job Category } & \multirow[b]{2}{*}{ TOTAL } \\
\hline & & Security and Fire & Crafts & Line Operators & \\
\hline Diagnostic Category & ICD-9-CM Code & \multirow[b]{2}{*}{0} & \multirow[b]{2}{*}{0} & \multirow[b]{2}{*}{6} & \multirow[b]{2}{*}{6} \\
\hline -Pain & 338 & & & & \\
\hline -Other Headache Syndromes & 339 & 0 & 0 & 1 & 1 \\
\hline -Other Disorders of Central NS & $340-349$ & 0 & 0 & 11 & 23 \\
\hline -Disorders of Peripheral NS & $350-359$ & 0 & 0 & 20 & 33 \\
\hline -Disorders of Eye & 360-379 & 0 & 12 & 9 & 84 \\
\hline -Diseases of Ear \& Mastoid & $380-389$ & 0 & 2 & 27 & 48 \\
\hline CIRCULATORY SYSTEM & $390-459$ & 1 & 21 & 90 & 280 \\
\hline -Hypertensive Dis & $401-405$ & 1 & 9 & 28 & 65 \\
\hline -Ischemic Heart Dis & $410-414$ & 0 & 3 & 20 & 77 \\
\hline -Dis of Pulmonary Circulation & $415-417$ & 0 & 0 & 3 & 7 \\
\hline -Other Heart Dis & $420-429$ & 0 & 2 & 12 & 54 \\
\hline -Cerebrovascular Dis & \begin{tabular}{|c|}
$430-438$ \\
\end{tabular} & 0 & 0 & 3 & 8 \\
\hline -Dis of Arteries \& Capillaries & $440-449$ & 0 & 3 & 3 & 11 \\
\hline -Dis of Veins, Lymphatics, Other & 451-459 & 0 & 4 & 21 & 58 \\
\hline RESPIRATORY SYSTEM & $460-519$ & 1 & 26 & 293 & 700 \\
\hline -Acute Respiratory Infections & \begin{tabular}{|c|}
$460-466$ \\
\end{tabular} & 1 & 14 & 178 & 406 \\
\hline -Other Dis Upper Respiratory Tract & $470-478$ & 0 & 2 & 44 & 115 \\
\hline -Pneumonia \& Influenza & $480-488$ & 0 & 8 & 34 & 97 \\
\hline -Chronic Obstructive Dis & $490-496$ & 0 & 2 & 25 & 60 \\
\hline -Lung Dis from External Agents & \begin{tabular}{|l|}
$500-508$ \\
\end{tabular} & 0 & 0 & 1 & 3 \\
\hline -Other Respiratory Dis & $510-519$ & 0 & 0 & 11 & 19 \\
\hline DIGESTIVE SYSTEM & $520-579$ & 0 & 19 & 72 & 259 \\
\hline -Oral Cavity, Saliva Glands, Jaw & $520-529$ & 0 & 3 & 13 & 31 \\
\hline -Esophagus, Stomach, Duodenum & $530-538$ & 0 & 4 & 13 & 44 \\
\hline -Appendicitis & $540-543$ & 0 & 1 & 1 & 7 \\
\hline -Hernias & $550-553$ & 0 & 3 & 8 & 55 \\
\hline -Enteritis, Colitis & $555-558$ & 0 & 0 & 7 & 16 \\
\hline -Other Intestinal Dis & $560-569$ & 0 & 6 & 15 & 68 \\
\hline -Other Digestive Dis & $570-579$ & 0 & 2 & 15 & 38 \\
\hline GENITOURINARY SYSTEM & $580-629$ & 0 & 7 & 53 & 141 \\
\hline -Nephritis, Nephrosis & $580-589$ & 0 & 0 & 2 & 4 \\
\hline -Other Urinary Dis & $590-599$ & 0 & 5 & 40 & 109 \\
\hline -Male Genital Organ Dis & $600-608$ & 0 & 2 & 11 & 28 \\
\hline SKIN AND SUBCUTANEOUS TISSUE & $680-709$ & 1 & 4 & 30 & 54 \\
\hline -Infections & $680-686$ & 1 & 2 & 15 & 28 \\
\hline -Other Inflammatory Conditions & 690-698 & 0 & 1 & 8 & 12 \\
\hline
\end{tabular}

(Continued)

*Only those diagnostic categories and gender/job category combinations with at least one occurrence appear in this table. 


\section{Savannah River Site 2010}

\section{Absence Data}

Appendix I. Number of Diagnoses in Each Diagnostic Category by Gender and Job Category*

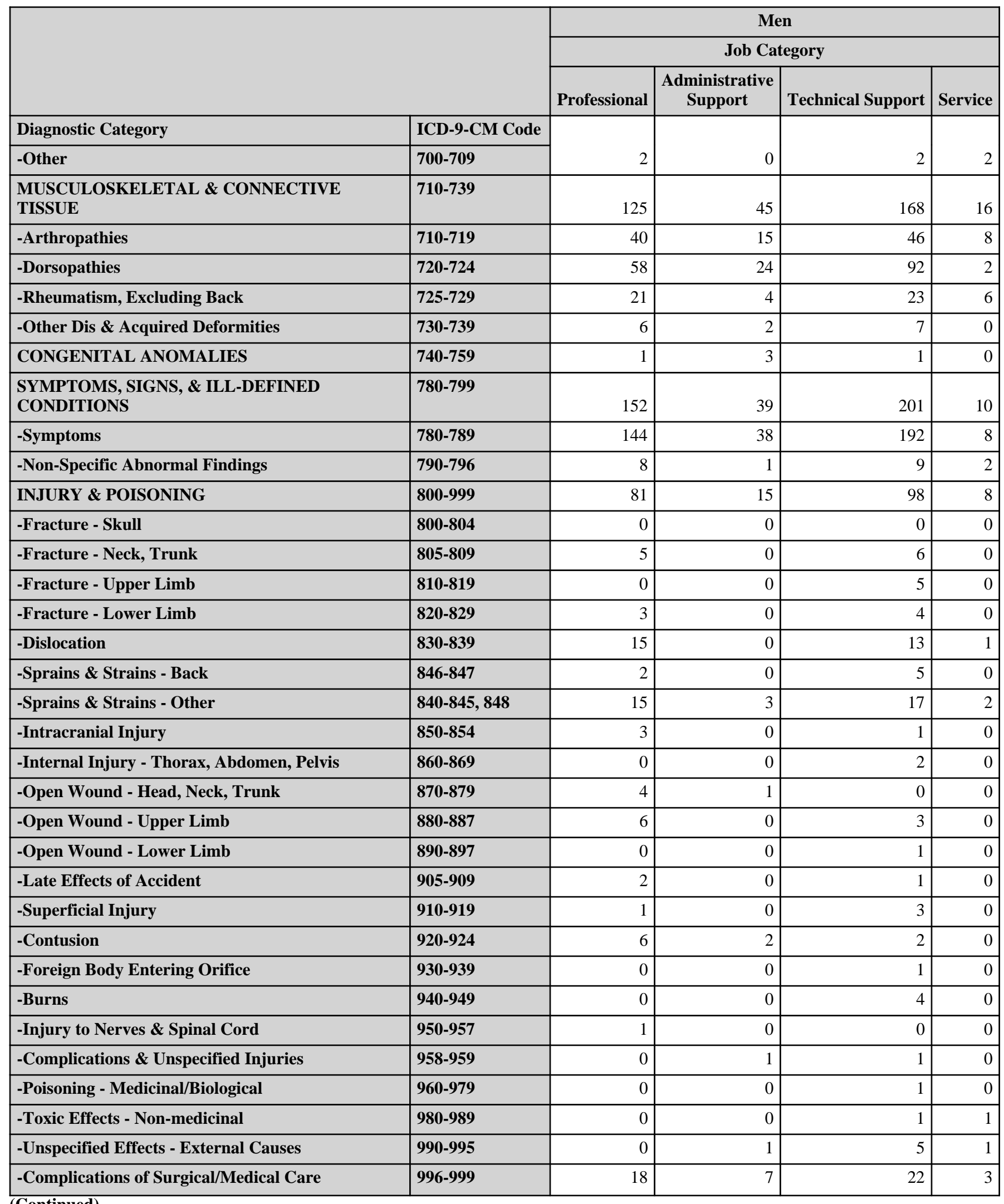

(Continued)

*Only those diagnostic categories and gender/job category combinations with at least one occurrence appear in this table. 


\section{Savannah River Site 2010}

\section{Absence Data}

Appendix I. Number of Diagnoses in Each Diagnostic Category by Gender and Job Category*

\begin{tabular}{|c|c|c|c|c|c|}
\hline & & \multicolumn{4}{|c|}{ Men } \\
\hline & & \multicolumn{3}{|c|}{ Job Category } & \multirow[b]{2}{*}{ TOTAL } \\
\hline & & Security and Fire & Crafts & Line Operators & \\
\hline Diagnostic Category & ICD-9-CM Code & \multirow[b]{2}{*}{0} & \multirow[b]{2}{*}{1} & \multirow[b]{2}{*}{7} & \multirow[b]{2}{*}{14} \\
\hline -Other & 700-709 & & & & \\
\hline $\begin{array}{l}\text { MUSCULOSKELETAL \& CONNECTIVE } \\
\text { TISSUE }\end{array}$ & 710-739 & 0 & 35 & 306 & 695 \\
\hline -Arthropathies & $710-719$ & 0 & 10 & 100 & 219 \\
\hline -Dorsopathies & $720-724$ & 0 & 21 & 139 & 336 \\
\hline -Rheumatism, Excluding Back & 725-729 & 0 & 3 & 60 & 117 \\
\hline -Other Dis \& Acquired Deformities & 730-739 & 0 & 1 & 7 & 23 \\
\hline CONGENITAL ANOMALIES & 740-759 & 0 & 0 & 1 & 6 \\
\hline $\begin{array}{l}\text { SYMPTOMS, SIGNS, \& ILL-DEFINED } \\
\text { CONDITIONS }\end{array}$ & 780-799 & 4 & 41 & 305 & 752 \\
\hline -Symptoms & 780-789 & 4 & 40 & 298 & 724 \\
\hline -Non-Specific Abnormal Findings & $790-796$ & 0 & 1 & 7 & 28 \\
\hline INJURY \& POISONING & 800-999 & 1 & 23 & 85 & 311 \\
\hline -Fracture - Skull & $800-804$ & 0 & 0 & 2 & 2 \\
\hline -Fracture - Neck, Trunk & 805-809 & 0 & 1 & 3 & 15 \\
\hline -Fracture - Upper Limb & 810-819 & 0 & 1 & 0 & 6 \\
\hline -Fracture - Lower Limb & $820-829$ & 1 & 0 & 4 & 12 \\
\hline -Dislocation & $830-839$ & 0 & 1 & 6 & 36 \\
\hline -Sprains \& Strains - Back & 846-847 & 0 & 3 & 14 & 24 \\
\hline -Sprains \& Strains - Other & $840-845,848$ & 0 & 7 & 19 & 63 \\
\hline -Intracranial Injury & $850-854$ & 0 & 2 & 1 & 7 \\
\hline -Internal Injury - Thorax, Abdomen, Pelvis & $860-869$ & 0 & 0 & 1 & 3 \\
\hline -Open Wound - Head, Neck, Trunk & $870-879$ & 0 & 2 & 1 & 8 \\
\hline -Open Wound - Upper Limb & $880-887$ & 0 & 2 & 1 & 12 \\
\hline -Open Wound - Lower Limb & 890-897 & 0 & 0 & 0 & 1 \\
\hline -Late Effects of Accident & 905-909 & 0 & 1 & 0 & 4 \\
\hline -Superficial Injury & 910-919 & 0 & 0 & 1 & 5 \\
\hline -Contusion & $920-924$ & 0 & 1 & 7 & 18 \\
\hline -Foreign Body Entering Orifice & 930-939 & 0 & 0 & 0 & 1 \\
\hline -Burns & 940-949 & 0 & 0 & 2 & 6 \\
\hline -Injury to Nerves \& Spinal Cord & $950-957$ & 0 & 0 & 1 & 2 \\
\hline -Complications \& Unspecified Injuries & 958-959 & 0 & 0 & 2 & 4 \\
\hline -Poisoning - Medicinal/Biological & $960-979$ & 0 & 0 & 2 & 3 \\
\hline -Toxic Effects - Non-medicinal & 980-989 & 0 & 0 & 0 & 2 \\
\hline -Unspecified Effects - External Causes & $990-995$ & 0 & 1 & 2 & 10 \\
\hline -Complications of Surgical/Medical Care & 996-999 & 0 & 1 & 16 & 67 \\
\hline
\end{tabular}

(Continued)

*Only those diagnostic categories and gender/job category combinations with at least one occurrence appear in this table. 
Savannah River Site 2010

Absence Data

Appendix I. Number of Diagnoses in Each Diagnostic Category by Gender and Job Category*

\begin{tabular}{|c|c|c|c|c|c|}
\hline & & \multicolumn{4}{|c|}{ Men } \\
\hline & & \multicolumn{4}{|c|}{ Job Category } \\
\hline & & Professional & \begin{tabular}{c|} 
Administrative \\
Support
\end{tabular} & Technical Support & Service \\
\hline Diagnostic Category & ICD-9-CM Code & & & & \\
\hline $\begin{array}{l}\text { HEALTH STATUS/HEALTH SERVICE } \\
\text { CONTACT }\end{array}$ & V01-V89 & 14 & 8 & 14 & 2 \\
\hline -Personal \& Family History & V10-V19 & 1 & 0 & 0 & 2 \\
\hline -Health Services Reproduction/Development & V20-V29 & 1 & 1 & 1 & 0 \\
\hline -Health Status & V40-V49 & 0 & 1 & 3 & 0 \\
\hline -Specific Procedures/Aftercare & V50-V59 & 7 & 6 & 7 & 0 \\
\hline -Other Circumstances & V60-V69 & 4 & 0 & 1 & 0 \\
\hline -Examination \& Investigation & V70-V82 & 0 & 0 & 2 & 0 \\
\hline -Retained Foreign Body & V90 & 1 & 0 & 0 & 0 \\
\hline
\end{tabular}

*Only those diagnostic categories and gender/job category combinations with at least one occurrence appear in this table. 
Savannah River Site 2010

Absence Data

Appendix I. Number of Diagnoses in Each Diagnostic Category by Gender and Job Category*

\begin{tabular}{|c|c|c|c|c|c|}
\hline & & \multicolumn{4}{|c|}{ Men } \\
\hline & & \multicolumn{3}{|c|}{ Job Category } & \multirow[b]{2}{*}{ TOTAL } \\
\hline & & Security and Fire & Crafts & Line Operators & \\
\hline Diagnostic Category & ICD-9-CM Code & & & & \\
\hline $\begin{array}{l}\text { HEALTH STATUS/HEALTH SERVICE } \\
\text { CONTACT }\end{array}$ & \begin{tabular}{|l|} 
V01-V89 \\
\end{tabular} & 0 & 3 & 16 & 57 \\
\hline -Personal \& Family History & V10-V19 & 0 & 1 & 7 & 11 \\
\hline -Health Services Reproduction/Development & V20-V29 & 0 & 1 & 0 & 4 \\
\hline -Health Status & V40-V49 & 0 & 1 & 1 & 6 \\
\hline -Specific Procedures/Aftercare & V50-V59 & 0 & 0 & 5 & 25 \\
\hline -Other Circumstances & V60-V69 & 0 & 0 & 0 & 5 \\
\hline -Examination \& Investigation & V70-V82 & 0 & 0 & 3 & 5 \\
\hline -Retained Foreign Body & V90 & 0 & 0 & 0 & 1 \\
\hline
\end{tabular}

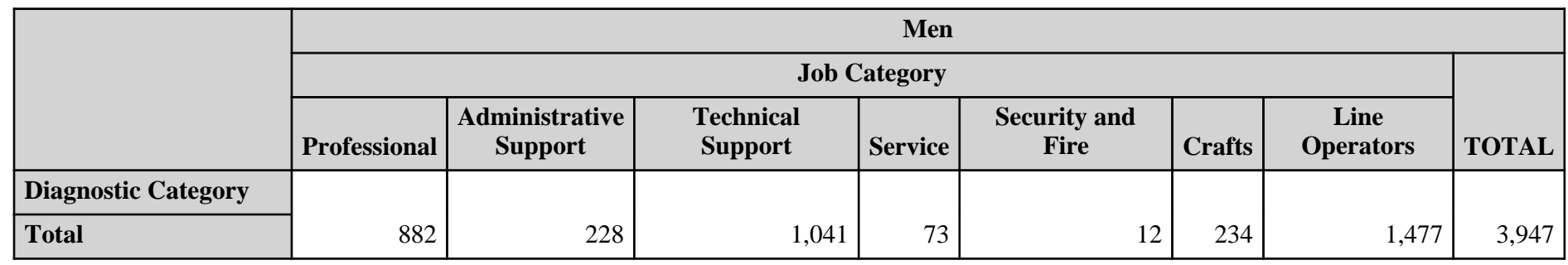

*Only those diagnostic categories and gender/job category combinations with at least one occurrence appear in this table. 


\section{Savannah River Site 2010}

\section{Absence Data}

Appendix J. Total Number of Calendar Days Absent in Each Diagnostic Category by Gender and Job Category*

\begin{tabular}{|c|c|c|c|c|}
\hline & & \multicolumn{3}{|c|}{ Women } \\
\hline & & \multicolumn{3}{|c|}{ Job Category } \\
\hline & & Professional & $\begin{array}{l}\text { Administrative } \\
\text { Support }\end{array}$ & Technical Support \\
\hline Diagnostic Category & ICD-9-CM Code & \multirow[b]{2}{*}{77} & \multirow[b]{2}{*}{196} & \multirow[b]{2}{*}{250} \\
\hline INFECTIOUS \& PARASITIC DISEASES (DIS) & 001-139 & & & \\
\hline MALIGNANT NEOPLASMS & $\begin{array}{l}140-208, \\
209.0-209.3 \\
230-234\end{array}$ & 273 & 393 & 225 \\
\hline BENIGN \& UNCERTAIN NEOPLASMS & $\begin{array}{l}209.4-209.6 \\
210-229,235-239\end{array}$ & 392 & 220 & 294 \\
\hline ENDOCRINE/METABOLIC/IMMUNITY & $240-279$ & 125 & 183 & 217 \\
\hline BLOOD \& BLOOD-FORMING ORGANS & $280-289$ & 60 & 90 & 83 \\
\hline MENTAL DISORDERS & 290-319 & 55 & 222 & 71 \\
\hline NERVOUS SYSTEM (NS) \& SENSE ORGANS & $320-389$ & 135 & 560 & 504 \\
\hline CIRCULATORY SYSTEM & $390-459$ & 321 & 53 & 154 \\
\hline RESPIRATORY SYSTEM & $460-519$ & 603 & 552 & 1,039 \\
\hline DIGESTIVE SYSTEM & $520-579$ & 228 & 350 & 446 \\
\hline GENITOURINARY SYSTEM & $580-629$ & 299 & 516 & 772 \\
\hline SKIN AND SUBCUTANEOUS TISSUE & $680-709$ & 44 & 34 & 212 \\
\hline $\begin{array}{l}\text { MUSCULOSKELETAL \& CONNECTIVE } \\
\text { TISSUE }\end{array}$ & 710-739 & 727 & 1,369 & 1,604 \\
\hline CONGENITAL ANOMALIES & $740-759$ & 0 & 14 & 0 \\
\hline $\begin{array}{l}\text { SYMPTOMS, SIGNS, \& ILL-DEFINED } \\
\text { CONDITIONS }\end{array}$ & \begin{tabular}{|l|}
$780-799$ \\
\end{tabular} & 371 & 818 & 1,245 \\
\hline INJURY \& POISONING & $800-999$ & 453 & 458 & 811 \\
\hline
\end{tabular}

\footnotetext{
*Absences with >1 ICD-9-CM code in the same diagnostic category were counted only once. Only those diagnostic categories and gender/job category combinations with at least one occurrence appear in this table.
} 
Savannah River Site 2010

Absence Data

Appendix J. Total Number of Calendar Days Absent in Each Diagnostic Category by Gender and Job Category*

\begin{tabular}{|c|c|c|c|c|c|}
\hline & & \multicolumn{4}{|c|}{ Women } \\
\hline & & \multicolumn{3}{|c|}{ Job Category } & \multirow[b]{2}{*}{ TOTAL } \\
\hline & & Service & Crafts & Line Operators & \\
\hline Diagnostic Category & ICD-9-CM Code & \multirow[b]{2}{*}{0} & \multirow[b]{2}{*}{0} & \multirow[b]{2}{*}{273} & \multirow[b]{2}{*}{796} \\
\hline INFECTIOUS \& PARASITIC DISEASES (DIS) & 001-139 & & & & \\
\hline MALIGNANT NEOPLASMS & $\begin{array}{l}\text { 140-208, } \\
209.0-209.3 \\
230-234\end{array}$ & 40 & 0 & 285 & 1,216 \\
\hline BENIGN \& UNCERTAIN NEOPLASMS & $\begin{array}{l}\text { 209.4-209.6, } \\
210-229,235-239\end{array}$ & 0 & 9 & 55 & 970 \\
\hline ENDOCRINE/METABOLIC/IMMUNITY & 240-279 & 0 & 0 & 164 & 689 \\
\hline BLOOD \& BLOOD-FORMING ORGANS & $280-289$ & 0 & 0 & 10 & 243 \\
\hline MENTAL DISORDERS & 290-319 & 0 & 13 & 126 & 487 \\
\hline NERVOUS SYSTEM (NS) \& SENSE ORGANS & 320-389 & 0 & 20 & 292 & 1,511 \\
\hline CIRCULATORY SYSTEM & 390-459 & 0 & 120 & 718 & 1,366 \\
\hline RESPIRATORY SYSTEM & $460-519$ & 0 & 33 & 753 & 2,980 \\
\hline DIGESTIVE SYSTEM & 520-579 & 39 & 26 & 481 & 1,570 \\
\hline GENITOURINARY SYSTEM & 580-629 & 0 & 127 & 208 & 1,922 \\
\hline SKIN AND SUBCUTANEOUS TISSUE & 680-709 & 9 & 0 & 118 & 417 \\
\hline $\begin{array}{l}\text { MUSCULOSKELETAL \& CONNECTIVE } \\
\text { TISSUE }\end{array}$ & 710-739 & 14 & 387 & 1,092 & 5,193 \\
\hline CONGENITAL ANOMALIES & 740-759 & 0 & 0 & 193 & 207 \\
\hline $\begin{array}{l}\text { SYMPTOMS, SIGNS, \& ILL-DEFINED } \\
\text { CONDITIONS }\end{array}$ & 780-799 & 40 & 162 & 861 & 3,497 \\
\hline INJURY \& POISONING & $800-999$ & 0 & 270 & 628 & 2,620 \\
\hline
\end{tabular}

\footnotetext{
*Absences with >1 ICD-9-CM code in the same diagnostic category were counted only once. Only those diagnostic categories and gender/job
} category combinations with at least one occurrence appear in this table. 
Savannah River Site 2010

Absence Data

Appendix J. Total Number of Calendar Days Absent in Each Diagnostic Category by Gender and Job Category*

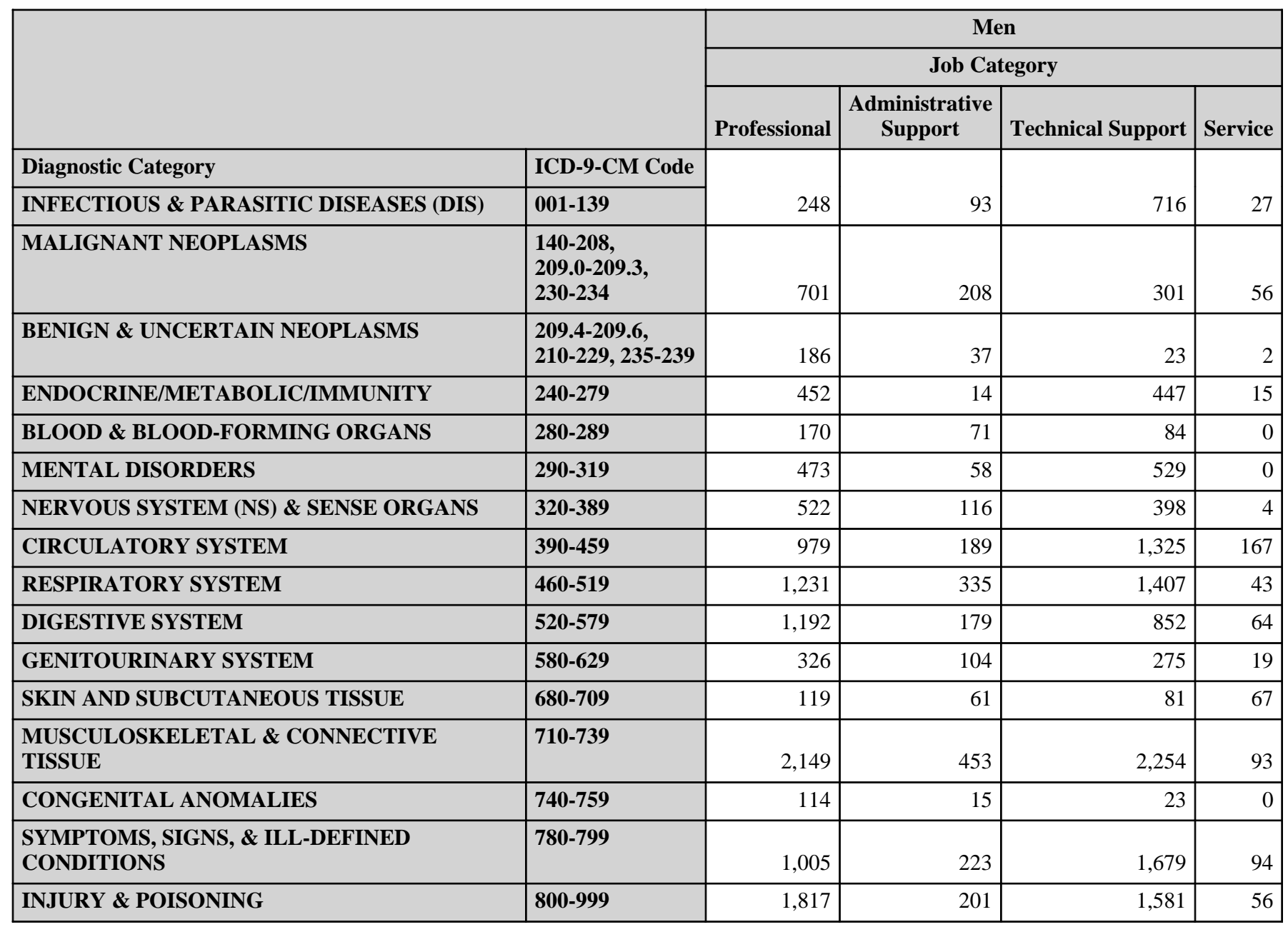

\footnotetext{
*Absences with >1 ICD-9-CM code in the same diagnostic category were counted only once. Only those diagnostic categories and gender/job category combinations with at least one occurrence appear in this table.
} 
Savannah River Site 2010

Absence Data

Appendix J. Total Number of Calendar Days Absent in Each Diagnostic Category by Gender and Job Category*

\begin{tabular}{|c|c|c|c|c|c|}
\hline & & \multicolumn{4}{|c|}{ Men } \\
\hline & & \multicolumn{3}{|c|}{ Job Category } & \multirow[b]{2}{*}{ TOTAL } \\
\hline & & Security and Fire & Crafts & Line Operators & \\
\hline Diagnostic Category & ICD-9-CM Code & \multirow[b]{2}{*}{32} & \multirow[b]{2}{*}{53} & \multirow[b]{2}{*}{430} & \multirow[b]{2}{*}{1,599} \\
\hline INFECTIOUS \& PARASITIC DISEASES (DIS) & 001-139 & & & & \\
\hline MALIGNANT NEOPLASMS & $\begin{array}{l}140-208 \\
209.0-209.3 \\
230-234\end{array}$ & 0 & 170 & 267 & 1,703 \\
\hline BENIGN \& UNCERTAIN NEOPLASMS & $\begin{array}{l}209.4-209.6 \\
210-229,235-239\end{array}$ & 0 & 49 & 160 & 457 \\
\hline ENDOCRINE/METABOLIC/IMMUNITY & $240-279$ & 0 & 71 & 877 & 1,876 \\
\hline BLOOD \& BLOOD-FORMING ORGANS & 280-289 & 0 & 0 & 159 & 484 \\
\hline MENTAL DISORDERS & 290-319 & 8 & 120 & 185 & 1,373 \\
\hline NERVOUS SYSTEM (NS) \& SENSE ORGANS & 320-389 & 0 & 89 & 993 & 2,122 \\
\hline CIRCULATORY SYSTEM & $390-459$ & 8 & 262 & 1,160 & 4,090 \\
\hline RESPIRATORY SYSTEM & $460-519$ & 8 & 167 & 2,478 & 5,669 \\
\hline DIGESTIVE SYSTEM & $520-579$ & 0 & 175 & 841 & 3,303 \\
\hline GENITOURINARY SYSTEM & $580-629$ & 0 & 101 & 596 & 1,421 \\
\hline SKIN AND SUBCUTANEOUS TISSUE & $680-709$ & 24 & 15 & 379 & 746 \\
\hline $\begin{array}{l}\text { MUSCULOSKELETAL \& CONNECTIVE } \\
\text { TISSUE }\end{array}$ & $710-739$ & 0 & 597 & 3,188 & 8,734 \\
\hline CONGENITAL ANOMALIES & $740-759$ & 0 & 0 & 18 & 170 \\
\hline $\begin{array}{l}\text { SYMPTOMS, SIGNS, \& ILL-DEFINED } \\
\text { CONDITIONS }\end{array}$ & \begin{tabular}{|l|}
$780-799$ \\
\end{tabular} & 13 & 310 & 2,237 & 5,561 \\
\hline INJURY \& POISONING & $800-999$ & 11 & 334 & 1,696 & 5,696 \\
\hline
\end{tabular}

\footnotetext{
*Absences with >1 ICD-9-CM code in the same diagnostic category were counted only once. Only those diagnostic categories and gender/job category combinations with at least one occurrence appear in this table.
} 


\section{Savannah River Site 2010}

\section{Absence Data}

Appendix K. Age-Adjusted IIIness and Injury Rates by Diagnostic Category*

Part 1. Men

\begin{tabular}{|c|c|c|c|c|c|}
\hline & & $\begin{array}{l}\text { Number of } \\
\text { Diagnoses }\end{array}$ & $\begin{array}{c}\text { Age-Adjusted } \\
\text { Rate per } 1,000 * *\end{array}$ & $\begin{array}{c}\text { Lower } 95 \% \\
\text { Confidence } \\
\text { Limit per 1,000 }\end{array}$ & $\begin{array}{c}\text { Upper } 95 \% \\
\text { Confidence } \\
\text { Limit per 1,000 }\end{array}$ \\
\hline Diagnostic Category & ICD-9-CM Code & \multirow[b]{2}{*}{140} & \multirow[b]{2}{*}{19.8} & \multirow[b]{2}{*}{15.7} & \multirow[b]{2}{*}{24.8} \\
\hline INFECTIOUS \& PARASITIC DISEASES (DIS) & 001-139 & & & & \\
\hline -Intestinal Infectious Dis & 001-009 & 78 & 11.2 & 8.2 & 15.3 \\
\hline -Other Bacterial Dis & 030-041 & 32 & 4.4 & 2.8 & 7.1 \\
\hline -HIV & 042 & 1 & 0.1 & 0.0 & 0.8 \\
\hline -Viral Dis with Exanthem & 050-059 & 17 & 2.1 & 1.1 & 4.2 \\
\hline -Other Viral Dis \& Chlamydiae & 070-079 & 9 & 1.7 & 0.7 & 3.9 \\
\hline -Rickettsioses \& Other Arthropod & 080-088 & 1 & 0.0 & 0.0 & 0.3 \\
\hline -Mycoses & $110-118$ & 1 & 0.1 & 0.0 & 0.8 \\
\hline -Other Infections \& Parasitic Dis & $130-136$ & 1 & 0.0 & 0.0 & 0.3 \\
\hline MALIGNANT NEOPLASMS & $\begin{array}{l}140-208, \\
209.0-209.3, \\
230-234\end{array}$ & 76 & 5.1 & 3.9 & 6.7 \\
\hline -Lip, Oral Cavity, Pharynx & $140-149$ & 5 & 0.4 & 0.1 & 1.0 \\
\hline -Digestive \& Peritoneal & 150-159 & 5 & 0.3 & 0.1 & 0.8 \\
\hline -Respiratory \& Intrathoracic & $160-165$ & 3 & 0.3 & 0.1 & 0.9 \\
\hline -Bone, Connective Tissue, Skin & 170-173, 176 & 18 & 1.3 & 0.8 & 2.1 \\
\hline -Genitourinary & 179-189 & 17 & 0.9 & 0.5 & 1.5 \\
\hline -Endocrine & $\begin{array}{l}\text { 193-194, } \\
209.0-209.3\end{array}$ & 7 & 0.8 & 0.3 & 2.4 \\
\hline -Other \& Unspecified Sites & 195-199, 209.7 & 14 & 0.7 & 0.4 & 1.2 \\
\hline -Lymphatic \& Hematopoietic & $200-208$ & 7 & 0.5 & 0.2 & 1.1 \\
\hline BENIGN \& UNCERTAIN NEOPLASMS & $\begin{array}{l}209.4-209.6 \\
210-229,235-239\end{array}$ & 29 & 2.8 & 1.7 & 4.8 \\
\hline ENDOCRINE/METABOLIC/IMMUNITY & $240-279$ & 132 & 12.9 & 10.4 & 15.9 \\
\hline -Thyroid Gland Disorders & $240-246$ & 14 & 1.7 & 0.9 & 3.3 \\
\hline -Other Endocrine Gland Dis & $249-259$ & 45 & 4.1 & 3.0 & 5.6 \\
\hline -Other Metabolic \& Immunity Disorders & 270-279 & 73 & 7.0 & 5.2 & 9.5 \\
\hline BLOOD \& BLOOD-FORMING ORGANS & $280-289$ & 29 & 2.5 & 1.7 & 3.8 \\
\hline MENTAL DISORDERS & 290-319 & 84 & 11.4 & 8.4 & 15.4 \\
\hline -Psychoses & 290-299 & 14 & 1.9 & 0.9 & 4.0 \\
\hline -Non-Psychotic Disorders & $300-302,306-316$ & 57 & 7.4 & 5.3 & 10.5 \\
\hline -Alcohol Dependence & 303 & 1 & 0.5 & 0.1 & 3.6 \\
\hline -Drug Dependence & $304-305$ & 12 & 1.6 & 0.6 & 3.9 \\
\hline NERVOUS SYSTEM (NS) \& SENSE ORGANS & 320-389 & 202 & 21.3 & 17.7 & 25.6 \\
\hline -Inflammatory Dis of Central NS & $320-326$ & 1 & 0.1 & 0.0 & 0.8 \\
\hline -Organic Sleep Disorders & 327 & 3 & 0.6 & 0.1 & 2.6 \\
\hline -Hereditary/Degenerative Central NS Dis & 330-337 & 3 & 0.3 & 0.1 & 0.9 \\
\hline
\end{tabular}

(Continued)

* Only those diagnostic categories with at least one occurrence appear in this table.

**Standardized to age distribution of 2000 U.S. population. 


\section{Savannah River Site 2010}

\section{Absence Data}

Appendix K. Age-Adjusted IIIness and Injury Rates by Diagnostic Category*

Part 1. Men

\begin{tabular}{|c|c|c|c|c|c|}
\hline & & $\begin{array}{l}\text { Number of } \\
\text { Diagnoses }\end{array}$ & $\begin{array}{c}\text { Age-Adjusted } \\
\text { Rate per } 1,000 * *\end{array}$ & $\begin{array}{c}\text { Lower } 95 \% \\
\text { Confidence } \\
\text { Limit per 1,000 }\end{array}$ & $\begin{array}{c}\text { Upper } 95 \% \\
\text { Confidence } \\
\text { Limit per 1,000 }\end{array}$ \\
\hline Diagnostic Category & ICD-9-CM Code & \multirow[b]{2}{*}{6} & \multirow[b]{2}{*}{0.3} & \multirow[b]{2}{*}{0.1} & \multirow[b]{2}{*}{0.7} \\
\hline -Pain & 338 & & & & \\
\hline -Other Headache Syndromes & 339 & 1 & 0.1 & 0.0 & 0.8 \\
\hline -Other Disorders of Central NS & $340-349$ & 23 & 1.7 & 1.1 & 2.7 \\
\hline -Disorders of Peripheral NS & $350-359$ & 33 & 3.6 & 2.1 & 5.9 \\
\hline -Disorders of Eye & $360-379$ & 84 & 9.7 & 7.3 & 12.8 \\
\hline -Diseases of Ear \& Mastoid & $380-389$ & 48 & 5.0 & 3.5 & 7.1 \\
\hline CIRCULATORY SYSTEM & $390-459$ & 280 & 21.7 & 18.9 & 24.8 \\
\hline -Hypertensive Dis & $401-405$ & 65 & 5.3 & 3.9 & 7.3 \\
\hline -Ischemic Heart Dis & $410-414$ & 77 & 5.7 & 4.5 & 7.3 \\
\hline -Dis of Pulmonary Circulation & $415-417$ & 7 & 0.5 & 0.2 & 1.1 \\
\hline -Other Heart Dis & $420-429$ & 54 & 4.4 & 3.3 & 5.9 \\
\hline -Cerebrovascular Dis & $430-438$ & 8 & 0.7 & 0.3 & 1.4 \\
\hline -Dis of Arteries \& Capillaries & $440-449$ & 11 & 0.7 & 0.4 & 1.2 \\
\hline -Dis of Veins, Lymphatics, Other & 451-459 & 58 & 4.4 & 3.2 & 6.0 \\
\hline RESPIRATORY SYSTEM & $460-519$ & 700 & 70.6 & 63.8 & 78.1 \\
\hline -Acute Respiratory Infections & $460-466$ & 406 & 41.9 & 36.6 & 47.9 \\
\hline -Other Dis Upper Respiratory Tract & $470-478$ & 115 & 11.1 & 8.8 & 14.1 \\
\hline -Pneumonia \& Influenza & $480-488$ & 97 & 9.0 & 6.9 & 11.8 \\
\hline -Chronic Obstructive Dis & $490-496$ & 60 & 6.5 & 4.6 & 9.1 \\
\hline -Lung Dis from External Agents & $500-508$ & 3 & 0.2 & 0.1 & 0.7 \\
\hline -Other Respiratory Dis & $510-519$ & 19 & 1.9 & 1.2 & 3.0 \\
\hline DIGESTIVE SYSTEM & $520-579$ & 259 & 24.9 & 20.9 & 29.7 \\
\hline -Oral Cavity, Saliva Glands, Jaw & $520-529$ & 31 & 3.1 & 1.8 & 5.4 \\
\hline -Esophagus, Stomach, Duodenum & $530-538$ & 44 & 4.4 & 2.9 & 6.7 \\
\hline -Appendicitis & $540-543$ & 7 & 0.5 & 0.2 & 1.1 \\
\hline -Hernias & $550-553$ & 55 & 4.7 & 3.4 & 6.5 \\
\hline -Enteritis, Colitis & $555-558$ & 16 & 1.7 & 0.8 & 3.4 \\
\hline -Other Intestinal Dis & $560-569$ & 68 & 7.6 & 5.3 & 11.0 \\
\hline -Other Digestive Dis & $570-579$ & 38 & 2.8 & 2.0 & 4.0 \\
\hline GENITOURINARY SYSTEM & $580-629$ & 141 & 13.5 & 10.8 & 16.9 \\
\hline -Nephritis, Nephrosis & $580-589$ & 4 & 0.3 & 0.1 & 0.8 \\
\hline -Other Urinary Dis & $590-599$ & 109 & 10.4 & 8.2 & 13.3 \\
\hline -Male Genital Organ Dis & \begin{tabular}{|c|}
$600-608$ \\
\end{tabular} & 28 & 2.8 & 1.6 & 5.0 \\
\hline SKIN AND SUBCUTANEOUS TISSUE & 680-709 & 54 & 6.2 & 4.2 & 9.1 \\
\hline -Infections & $680-686$ & 28 & 3.2 & 1.8 & 5.6 \\
\hline
\end{tabular}

(Continued)

*Only those diagnostic categories with at least one occurrence appear in this table.

**Standardized to age distribution of 2000 U.S. population. 


\section{Savannah River Site 2010}

\section{Absence Data}

Appendix K. Age-Adjusted IIIness and Injury Rates by Diagnostic Category*

Part 1. Men

\begin{tabular}{|c|c|c|c|c|c|}
\hline & & $\begin{array}{l}\text { Number of } \\
\text { Diagnoses }\end{array}$ & $\begin{array}{l}\text { Age-Adjusted } \\
\text { Rate per } 1,000 * *\end{array}$ & $\begin{array}{c}\text { Lower } 95 \% \\
\text { Confidence } \\
\text { Limit per } 1,000\end{array}$ & $\begin{array}{c}\text { Upper } 95 \% \\
\text { Confidence } \\
\text { Limit per 1,000 }\end{array}$ \\
\hline Diagnostic Category & ICD-9-CM Code & \multirow[b]{2}{*}{12} & \multirow[b]{2}{*}{1.6} & \multirow[b]{2}{*}{0.7} & \multirow[b]{2}{*}{3.3} \\
\hline -Other Inflammatory Conditions & $690-698$ & & & & \\
\hline -Other & $700-709$ & 14 & 1.4 & 0.7 & 2.9 \\
\hline $\begin{array}{l}\text { MUSCULOSKELETAL \& CONNECTIVE } \\
\text { TISSUE }\end{array}$ & 710-739 & 695 & 65.9 & 59.7 & 72.7 \\
\hline -Arthropathies & $710-719$ & 219 & 19.9 & 16.7 & 23.8 \\
\hline -Dorsopathies & $720-724$ & 336 & 31.8 & 27.6 & 36.6 \\
\hline -Rheumatism, Excluding Back & $725-729$ & 117 & 11.5 & 9.0 & 14.6 \\
\hline -Other Dis \& Acquired Deformities & $730-739$ & 23 & 2.7 & 1.6 & 4.6 \\
\hline CONGENITAL ANOMALIES & \begin{tabular}{|l|}
$740-759$ \\
\end{tabular} & 6 & 0.6 & 0.3 & 1.4 \\
\hline $\begin{array}{l}\text { SYMPTOMS, SIGNS, \& ILL-DEFINED } \\
\text { CONDITIONS }\end{array}$ & \begin{tabular}{|l|}
$780-799$ \\
\end{tabular} & 752 & 83.7 & 75.5 & 92.7 \\
\hline -Symptoms & $780-789$ & 724 & 81.7 & 73.6 & 90.7 \\
\hline -Non-Specific Abnormal Findings & $790-796$ & 28 & 2.0 & 1.3 & 3.0 \\
\hline INJURY \& POISONING & 800-999 & 311 & 34.5 & 29.5 & 40.4 \\
\hline -Fracture - Skull & $800-804$ & 17 & 2.1 & 1.1 & 4.4 \\
\hline -Fracture - Upper Limb & $810-819$ & 6 & 0.9 & 0.3 & 2.9 \\
\hline -Fracture - Lower Limb & $820-829$ & 12 & 1.6 & 0.7 & 3.6 \\
\hline -Dislocation & $830-839$ & 36 & 3.2 & 2.1 & 4.7 \\
\hline -Sprains \& Strains - Back & 846-847 & 24 & 3.0 & 1.7 & 5.3 \\
\hline -Sprains \& Strains - Other & $840-845,848$ & 63 & 7.6 & 5.4 & 10.8 \\
\hline -Intracranial Injury & $850-854$ & 7 & 1.0 & 0.3 & 2.9 \\
\hline -Internal Injury - Thorax, Abdomen, Pelvis & $860-869$ & 3 & 0.3 & 0.1 & 0.9 \\
\hline -Open Wound - Head, Neck, Trunk & $870-879$ & 21 & 2.1 & 1.2 & 3.7 \\
\hline -Late Effects of Accident & $905-909$ & 4 & 0.7 & 0.2 & 3.0 \\
\hline -Superficial Injury & $910-919$ & 5 & 0.4 & 0.1 & 1.0 \\
\hline -Contusion & $920-924$ & 18 & 3.5 & 1.8 & 6.7 \\
\hline -Foreign Body Entering Orifice & 930-939 & 1 & 0.0 & 0.0 & 0.3 \\
\hline -Burns & $940-949$ & 6 & 0.4 & 0.2 & 1.0 \\
\hline -Injury to Nerves \& Spinal Cord & $950-957$ & 2 & 0.2 & 0.0 & 0.7 \\
\hline -Complications \& Unspecified Injuries & 958-959 & 4 & 0.3 & 0.1 & 0.9 \\
\hline -Poisoning - Medicinal/Biological & $960-979$ & 3 & 0.1 & 0.0 & 0.5 \\
\hline -Toxic Effects - Non-medicinal & $980-989$ & 2 & 0.2 & 0.0 & 0.7 \\
\hline -Unspecified Effects - External Causes & $990-995$ & 10 & 0.7 & 0.3 & 1.4 \\
\hline -Complications of Surgical/Medical Care & 996-999 & 67 & 6.1 & 4.6 & 8.2 \\
\hline Total & & 3890 & 397.4 & 380.6 & 415.1 \\
\hline
\end{tabular}

*Only those diagnostic categories with at least one occurrence appear in this table.

**Standardized to age distribution of 2000 U.S. population. 


\section{Savannah River Site 2010}

\section{Absence Data}

Appendix K. Age-Adjusted Illness and Injury Rates by Diagnostic Category*

Part 2. Women

\begin{tabular}{|c|c|c|c|c|c|}
\hline & & $\begin{array}{l}\text { Number of } \\
\text { Diagnoses }\end{array}$ & $\begin{array}{c}\text { Age-Adjusted } \\
\text { Rate per } 1,000 * *\end{array}$ & $\begin{array}{c}\text { Lower } 95 \% \\
\text { Confidence } \\
\text { Limit per 1,000 }\end{array}$ & $\begin{array}{c}\text { Upper } 95 \% \\
\text { Confidence } \\
\text { Limit per 1,000 }\end{array}$ \\
\hline Diagnostic Category & ICD-9-CM Code & \multirow[b]{2}{*}{69} & \multirow[b]{2}{*}{24.6} & \multirow[b]{2}{*}{17.9} & \multirow[b]{2}{*}{33.9} \\
\hline INFECTIOUS \& PARASITIC DISEASES (DIS) & 001-139 & & & & \\
\hline -Intestinal Infectious Dis & 001-009 & 29 & 13.0 & 7.9 & 21.3 \\
\hline -Other Bacterial Dis & $030-041$ & 15 & 4.1 & 2.3 & 7.4 \\
\hline -Viral Dis with Exanthem & 050-059 & 8 & 1.8 & 0.8 & 4.2 \\
\hline -Other Viral Dis \& Chlamydiae & 070-079 & 13 & 4.8 & 2.4 & 9.8 \\
\hline -Syphilis \& Other Venereal Dis & 090-099 & 1 & 0.3 & 0.0 & 2.4 \\
\hline -Mycoses & $110-118$ & 3 & 0.5 & 0.2 & 1.6 \\
\hline MALIGNANT NEOPLASMS & $\begin{array}{l}140-208, \\
209.0-209.3 \\
230-234\end{array}$ & 64 & 19.6 & 14.7 & 26.1 \\
\hline -Lip, Oral Cavity, Pharynx & 140-149 & 2 & 0.3 & 0.1 & 1.3 \\
\hline -Digestive \& Peritoneal & $150-159$ & 2 & 0.7 & 0.2 & 2.7 \\
\hline -Respiratory \& Intrathoracic & $160-165$ & 6 & 1.9 & 0.8 & 4.2 \\
\hline -Bone, Connective Tissue, Skin & $170-173,176$ & 3 & 1.1 & 0.3 & 4.1 \\
\hline -Breast & 174-175 & 26 & 11.2 & 7.3 & 17.2 \\
\hline -Genitourinary & 179-189 & 7 & 1.3 & 0.6 & 2.9 \\
\hline -Endocrine & $\begin{array}{l}\text { 193-194, } \\
209.0-209.3\end{array}$ & 2 & 0.3 & 0.1 & 1.3 \\
\hline -Other \& Unspecified Sites & 195-199, 209.7 & 6 & 1.0 & 0.5 & 2.2 \\
\hline -Lymphatic \& Hematopoietic & $200-208$ & 10 & 1.7 & 0.9 & 3.1 \\
\hline BENIGN \& UNCERTAIN NEOPLASMS & $\begin{array}{l}209.4-209.6, \\
210-229,235-239\end{array}$ & 42 & 19.3 & 12.7 & 29.3 \\
\hline ENDOCRINE/METABOLIC/IMMUNITY & 240-279 & 46 & 13.8 & 9.7 & 19.7 \\
\hline -Thyroid Gland Disorders & $240-246$ & 7 & 3.6 & 1.4 & 9.1 \\
\hline -Other Endocrine Gland Dis & 249-259 & 19 & 4.8 & 2.9 & 8.1 \\
\hline -Other Metabolic \& Immunity Disorders & 270-279 & 20 & 5.4 & 3.3 & 8.7 \\
\hline BLOOD \& BLOOD-FORMING ORGANS & 280-289 & 23 & 8.0 & 4.6 & 14.0 \\
\hline MENTAL DISORDERS & 290-319 & 30 & 17.5 & 11.1 & 27.6 \\
\hline -Psychoses & 290-299 & 6 & 3.9 & 1.5 & 10.1 \\
\hline -Non-Psychotic Disorders & $300-302,306-316$ & 21 & 13.0 & 7.6 & 22.3 \\
\hline -Drug Dependence & 304-305 & 3 & 0.7 & 0.2 & 2.2 \\
\hline NERVOUS SYSTEM (NS) \& SENSE ORGANS & $320-389$ & 132 & 55.5 & 43.9 & 70.2 \\
\hline -Pain & 338 & 1 & 1.3 & 0.2 & 9.4 \\
\hline -Other Headache Syndromes & 339 & 3 & 0.7 & 0.2 & 2.2 \\
\hline -Other Disorders of Central NS & 340-349 & 37 & 20.2 & 13.0 & 31.4 \\
\hline -Disorders of Peripheral NS & 350-359 & 30 & 10.9 & 6.8 & 17.5 \\
\hline -Disorders of Eye & $360-379$ & 43 & 15.2 & 10.1 & 22.9 \\
\hline
\end{tabular}

(Continued)

* Only those diagnostic categories with at least one occurrence appear in this table.

**Standardized to age distribution of 2000 U.S. population. 


\section{Savannah River Site 2010}

\section{Absence Data}

Appendix K. Age-Adjusted IIIness and Injury Rates by Diagnostic Category*

Part 2. Women

\begin{tabular}{|c|c|c|c|c|c|}
\hline & & $\begin{array}{l}\text { Number of } \\
\text { Diagnoses }\end{array}$ & $\begin{array}{c}\text { Age-Adjusted } \\
\text { Rate per } 1,000 * *\end{array}$ & $\begin{array}{c}\text { Lower } 95 \% \\
\text { Confidence } \\
\text { Limit per 1,000 }\end{array}$ & $\begin{array}{c}\text { Upper } 95 \% \\
\text { Confidence } \\
\text { Limit per 1,000 }\end{array}$ \\
\hline Diagnostic Category & ICD-9-CM Code & \multirow[b]{2}{*}{18} & \multirow[b]{2}{*}{7.3} & \multirow[b]{2}{*}{4.1} & \multirow[b]{2}{*}{12.8} \\
\hline -Diseases of Ear \& Mastoid & 380-389 & & & & \\
\hline CIRCULATORY SYSTEM & $390-459$ & 77 & 26.0 & 19.4 & 34.9 \\
\hline -Hypertensive Dis & $401-405$ & 21 & 6.5 & 3.7 & 11.4 \\
\hline -Ischemic Heart Dis & $410-414$ & 14 & 3.9 & 2.2 & 6.9 \\
\hline -Dis of Pulmonary Circulation & $415-417$ & 1 & 0.2 & 0.0 & 1.2 \\
\hline -Other Heart Dis & $420-429$ & 11 & 3.7 & 1.9 & 7.1 \\
\hline -Cerebrovascular Dis & $430-438$ & 5 & 1.0 & 0.4 & 2.5 \\
\hline -Dis of Arteries \& Capillaries & $440-449$ & 3 & 2.4 & 0.6 & 9.6 \\
\hline -Dis of Veins, Lymphatics, Other & 451-459 & 22 & 8.5 & 4.9 & 14.7 \\
\hline RESPIRATORY SYSTEM & $460-519$ & 399 & 149.6 & 130.8 & 171.1 \\
\hline -Acute Respiratory Infections & $460-466$ & 239 & 89.5 & 74.9 & 106.8 \\
\hline -Other Dis Upper Respiratory Tract & $470-478$ & 67 & 27.2 & 19.8 & 37.3 \\
\hline -Pneumonia \& Influenza & $480-488$ & 52 & 17.1 & 11.9 & 24.5 \\
\hline -Chronic Obstructive Dis & $490-496$ & 32 & 13.2 & 8.4 & 20.8 \\
\hline -Lung Dis from External Agents & $500-508$ & 1 & 0.2 & 0.0 & 1.2 \\
\hline -Other Respiratory Dis & $510-519$ & 8 & 2.5 & 1.2 & 5.4 \\
\hline DIGESTIVE SYSTEM & $520-579$ & 130 & 59.9 & 47.3 & 75.7 \\
\hline -Oral Cavity, Saliva Glands, Jaw & $520-529$ & 30 & 21.1 & 13.3 & 33.4 \\
\hline -Esophagus, Stomach, Duodenum & $530-538$ & 27 & 9.6 & 5.6 & 16.5 \\
\hline -Appendicitis & $540-543$ & 4 & 0.8 & 0.3 & 2.4 \\
\hline -Hernias & $550-553$ & 16 & 7.3 & 4.0 & 13.4 \\
\hline -Enteritis, Colitis & $555-558$ & 9 & 2.5 & 1.2 & 5.3 \\
\hline -Other Intestinal Dis & $560-569$ & 17 & 6.9 & 3.7 & 12.7 \\
\hline -Other Digestive Dis & $570-579$ & 27 & 11.7 & 7.0 & 19.5 \\
\hline GENITOURINARY SYSTEM & $580-629$ & 146 & 63.7 & 50.9 & 79.8 \\
\hline -Nephritis, Nephrosis & \begin{tabular}{|c|}
$580-589$ \\
\end{tabular} & 5 & 1.2 & 0.5 & 3.0 \\
\hline -Other Urinary Dis & $590-599$ & 67 & 26.9 & 19.7 & 36.9 \\
\hline -Breast Disorders & $610-612$ & 8 & 1.3 & 0.7 & 2.7 \\
\hline -Pelvic Inflammatory Dis & 614-616 & 6 & 6.8 & 2.9 & 16.1 \\
\hline -Other Female Disorders & $617-629$ & 60 & 27.4 & 19.2 & 39.3 \\
\hline SKIN AND SUBCUTANEOUS TISSUE & 680-709 & 28 & 7.5 & 5.0 & 11.4 \\
\hline -Infections & $680-686$ & 10 & 3.4 & 1.7 & 6.5 \\
\hline -Other Inflammatory Conditions & 690-698 & 2 & 0.3 & 0.1 & 1.3 \\
\hline -Other & \begin{tabular}{|l|}
$700-709$ \\
\end{tabular} & 16 & 3.8 & 2.2 & 6.7 \\
\hline $\begin{array}{l}\text { MUSCULOSKELETAL \& CONNECTIVE } \\
\text { TISSUE }\end{array}$ & \begin{tabular}{|l|}
$710-739$ \\
\end{tabular} & 360 & 145.5 & 126.4 & 167.5 \\
\hline
\end{tabular}

(Continued)

* Only those diagnostic categories with at least one occurrence appear in this table.

**Standardized to age distribution of 2000 U.S. population. 


\section{Savannah River Site 2010}

\section{Absence Data}

Appendix K. Age-Adjusted IIIness and Injury Rates by Diagnostic Category*

Part 2. Women

\begin{tabular}{|c|c|c|c|c|c|}
\hline & & $\begin{array}{l}\text { Number of } \\
\text { Diagnoses }\end{array}$ & $\begin{array}{c}\text { Age-Adjusted } \\
\text { Rate per } 1,000 * *\end{array}$ & $\begin{array}{c}\text { Lower } 95 \% \\
\text { Confidence } \\
\text { Limit per } 1,000\end{array}$ & $\begin{array}{c}\text { Upper } 95 \% \\
\text { Confidence } \\
\text { Limit per 1,000 }\end{array}$ \\
\hline Diagnostic Category & ICD-9-CM Code & \multirow[b]{2}{*}{84} & \multirow[b]{2}{*}{35.1} & \multirow[b]{2}{*}{26.6} & \multirow[b]{2}{*}{46.3} \\
\hline -Arthropathies & $710-719$ & & & & \\
\hline -Dorsopathies & $720-724$ & 192 & 82.5 & 67.6 & 100.5 \\
\hline -Rheumatism, Excluding Back & $725-729$ & 70 & 23.0 & 17.2 & 30.8 \\
\hline -Other Dis \& Acquired Deformities & $730-739$ & 14 & 4.9 & 2.3 & 10.4 \\
\hline CONGENITAL ANOMALIES & $740-759$ & 3 & 1.1 & 0.3 & 4.1 \\
\hline $\begin{array}{l}\text { SYMPTOMS, SIGNS, \& ILL-DEFINED } \\
\text { CONDITIONS }\end{array}$ & \begin{tabular}{|l|}
$780-799$ \\
\end{tabular} & 427 & 177.1 & 155.3 & 201.9 \\
\hline -Symptoms & 780-789 & 414 & 170.0 & 148.9 & 194.2 \\
\hline -Non-Specific Abnormal Findings & $790-796$ & 13 & 7.1 & 3.3 & 15.1 \\
\hline INJURY \& POISONING & \begin{tabular}{|l|}
$800-999$ \\
\end{tabular} & 144 & 60.1 & 48.0 & 75.3 \\
\hline -Fracture - Skull & $800-804$ & 3 & 0.5 & 0.2 & 1.6 \\
\hline -Fracture - Upper Limb & 810-819 & 3 & 2.8 & 0.8 & 10.5 \\
\hline -Fracture - Lower Limb & \begin{tabular}{|l|}
$820-829$ \\
\end{tabular} & 9 & 2.7 & 0.9 & 7.5 \\
\hline -Dislocation & $830-839$ & 7 & 4.3 & 1.7 & 11.0 \\
\hline -Sprains \& Strains - Back & 846-847 & 19 & 6.4 & 3.6 & 11.6 \\
\hline -Sprains \& Strains - Other & $840-845,848$ & 27 & 10.2 & 6.1 & 16.9 \\
\hline -Intracranial Injury & $850-854$ & 1 & 0.3 & 0.0 & 2.4 \\
\hline -Open Wound - Head, Neck, Trunk & $870-879$ & 6 & 1.8 & 0.7 & 4.6 \\
\hline -Superficial Injury & 910-919 & 8 & 6.3 & 2.7 & 14.7 \\
\hline -Contusion & $920-924$ & 11 & 4.9 & 2.1 & 11.7 \\
\hline -Burns & $940-949$ & 6 & 2.0 & 0.9 & 4.5 \\
\hline -Injury to Nerves \& Spinal Cord & $950-957$ & 1 & 0.2 & 0.0 & 1.2 \\
\hline -Complications \& Unspecified Injuries & 958-959 & 4 & 1.0 & 0.4 & 2.9 \\
\hline -Poisoning - Medicinal/Biological & $960-979$ & 2 & 0.7 & 0.2 & 2.7 \\
\hline -Unspecified Effects - External Causes & $990-995$ & 7 & 3.7 & 1.4 & 9.7 \\
\hline -Complications of Surgical/Medical Care & 996-999 & 30 & 12.2 & 7.4 & 19.9 \\
\hline Total & & 2120 & 848.9 & 800.8 & 899.9 \\
\hline
\end{tabular}

*Only those diagnostic categories with at least one occurrence appear in this table.

**Standardized to age distribution of 2000 U.S. population. 


\section{Savannah River Site 2010}

\section{Absence Data}

Appendix K. Age-Adjusted IIIness and Injury Rates by Diagnostic Category*

Part 3. Men and Women

\begin{tabular}{|c|c|c|c|c|c|}
\hline & & $\begin{array}{l}\text { Number of } \\
\text { Diagnoses }\end{array}$ & $\begin{array}{c}\text { Age-Adjusted } \\
\text { Rate per 1,000** }\end{array}$ & $\begin{array}{c}\text { Lower } 95 \% \\
\text { Confidence } \\
\text { Limit per } 1,000\end{array}$ & $\begin{array}{c}\text { Upper } 95 \% \\
\text { Confidence } \\
\text { Limit per 1,000 }\end{array}$ \\
\hline Diagnostic Category & ICD-9-CM Code & & & & \\
\hline INFECTIOUS \& PARASITIC DISEASES (DIS) & 001-139 & 209 & 20.8 & 17.3 & 25.1 \\
\hline -Intestinal Infectious Dis & 001-009 & 107 & 11.7 & 9.0 & 15.1 \\
\hline -Other Bacterial Dis & 030-041 & 47 & 4.3 & 2.9 & 6.3 \\
\hline -HIV & 042 & 1 & 0.1 & 0.0 & 0.6 \\
\hline -Viral Dis with Exanthem & 050-059 & 25 & 2.0 & 1.1 & 3.5 \\
\hline -Other Viral Dis \& Chlamydiae & 070-079 & 22 & 2.4 & 1.4 & 4.2 \\
\hline -Rickettsioses \& Other Arthropod & 080-088 & 1 & 0.0 & 0.0 & 0.3 \\
\hline -Syphilis \& Other Venereal Dis & 090-099 & 1 & 0.1 & 0.0 & 0.6 \\
\hline -Mycoses & 110-118 & 4 & 0.2 & 0.1 & 0.6 \\
\hline -Other Infections \& Parasitic Dis & $130-136$ & 1 & 0.0 & 0.0 & 0.3 \\
\hline MALIGNANT NEOPLASMS & $\begin{array}{l}140-208, \\
209.0-209.3 \\
230-234\end{array}$ & 140 & 7.9 & 6.5 & 9.6 \\
\hline -Lip, Oral Cavity, Pharynx & $140-149$ & 7 & 0.4 & 0.2 & 0.9 \\
\hline -Digestive \& Peritoneal & 150-159 & 7 & 0.4 & 0.2 & 0.9 \\
\hline -Respiratory \& Intrathoracic & $160-165$ & 9 & 0.7 & 0.4 & 1.4 \\
\hline -Bone, Connective Tissue, Skin & $170-173,176$ & 21 & 1.2 & 0.8 & 1.9 \\
\hline -Breast & 174-175 & 26 & 2.0 & 1.3 & 3.0 \\
\hline -Genitourinary & 179-189 & 24 & 1.0 & 0.7 & 1.6 \\
\hline -Endocrine & $\begin{array}{l}\text { 193-194, } \\
209.0-209.3\end{array}$ & 9 & 0.7 & 0.2 & 1.8 \\
\hline -Other \& Unspecified Sites & 195-199, 209.7 & 20 & 0.8 & 0.5 & 1.2 \\
\hline -Lymphatic \& Hematopoietic & 200-208 & 17 & 0.7 & 0.5 & 1.2 \\
\hline BENIGN \& UNCERTAIN NEOPLASMS & $\begin{array}{l}209.4-209.6 \\
210-229,235-239\end{array}$ & 71 & 6.8 & 4.9 & 9.4 \\
\hline ENDOCRINE/METABOLIC/IMMUNITY & $240-279$ & 178 & 13.1 & 10.9 & 15.6 \\
\hline -Thyroid Gland Disorders & $240-246$ & 21 & 2.1 & 1.2 & 3.6 \\
\hline -Other Endocrine Gland Dis & 249-259 & 64 & 4.3 & 3.3 & 5.6 \\
\hline -Other Metabolic \& Immunity Disorders & 270-279 & 93 & 6.7 & 5.2 & 8.7 \\
\hline BLOOD \& BLOOD-FORMING ORGANS & $280-289$ & 52 & 3.9 & 2.8 & 5.4 \\
\hline MENTAL DISORDERS & 290-319 & 114 & 12.7 & 9.9 & 16.3 \\
\hline -Psychoses & 290-299 & 20 & 2.3 & 1.3 & 4.2 \\
\hline -Non-Psychotic Disorders & $300-302,306-316$ & 78 & 8.6 & 6.4 & 11.6 \\
\hline -Alcohol Dependence & 303 & 1 & 0.4 & 0.1 & 2.7 \\
\hline -Drug Dependence & 304-305 & 15 & 1.4 & 0.6 & 3.1 \\
\hline NERVOUS SYSTEM (NS) \& SENSE ORGANS & $320-389$ & 334 & 29.0 & 25.0 & 33.5 \\
\hline -Inflammatory Dis of Central NS & $320-326$ & 1 & 0.1 & 0.0 & 0.7 \\
\hline
\end{tabular}

(Continued)

* Only those diagnostic categories with at least one occurrence appear in this table.

**Standardized to age distribution of 2000 U.S. population. 


\section{Savannah River Site 2010}

\section{Absence Data}

Appendix K. Age-Adjusted IIIness and Injury Rates by Diagnostic Category*

Part 3. Men and Women

\begin{tabular}{|c|c|c|c|c|c|}
\hline & & $\begin{array}{l}\text { Number of } \\
\text { Diagnoses }\end{array}$ & $\begin{array}{c}\text { Age-Adjusted } \\
\text { Rate per } 1,000 * *\end{array}$ & $\begin{array}{c}\text { Lower } 95 \% \\
\text { Confidence } \\
\text { Limit per 1,000 }\end{array}$ & $\begin{array}{c}\text { Upper } 95 \% \\
\text { Confidence } \\
\text { Limit per 1,000 }\end{array}$ \\
\hline Diagnostic Category & ICD-9-CM Code & \multirow[b]{2}{*}{3} & \multirow[b]{2}{*}{0.5} & \multirow[b]{2}{*}{0.1} & \multirow[b]{2}{*}{1.9} \\
\hline -Organic Sleep Disorders & 327 & & & & \\
\hline -Hereditary/Degenerative Central NS Dis & 330-337 & 3 & 0.2 & 0.1 & 0.8 \\
\hline -Pain & 338 & 7 & 0.5 & 0.2 & 1.8 \\
\hline -Other Headache Syndromes & 339 & 4 & 0.3 & 0.1 & 0.7 \\
\hline -Other Disorders of Central NS & $340-349$ & 60 & 6.2 & 4.3 & 8.9 \\
\hline -Disorders of Peripheral NS & $350-359$ & 63 & 5.0 & 3.5 & 7.1 \\
\hline -Disorders of Eye & $360-379$ & 127 & 10.7 & 8.5 & 13.5 \\
\hline -Diseases of Ear \& Mastoid & 380-389 & 66 & 5.4 & 4.0 & 7.3 \\
\hline CIRCULATORY SYSTEM & $390-459$ & 357 & 23.4 & 20.6 & 26.6 \\
\hline -Hypertensive Dis & $401-405$ & 86 & 5.6 & 4.2 & 7.3 \\
\hline -Ischemic Heart Dis & $410-414$ & 91 & 5.6 & 4.5 & 7.1 \\
\hline -Dis of Pulmonary Circulation & $415-417$ & 8 & 0.4 & 0.2 & 0.9 \\
\hline -Other Heart Dis & $420-429$ & 65 & 4.5 & 3.5 & 5.9 \\
\hline -Cerebrovascular Dis & $430-438$ & 13 & 0.8 & 0.4 & 1.4 \\
\hline -Dis of Arteries \& Capillaries & $440-449$ & 14 & 1.1 & 0.5 & 2.3 \\
\hline -Dis of Veins, Lymphatics, Other & 451-459 & 80 & 5.4 & 4.1 & 7.2 \\
\hline RESPIRATORY SYSTEM & $460-519$ & 1099 & 88.3 & 81.4 & 95.7 \\
\hline -Acute Respiratory Infections & $460-466$ & 645 & 52.8 & 47.4 & 58.8 \\
\hline -Other Dis Upper Respiratory Tract & $470-478$ & 182 & 14.6 & 12.1 & 17.7 \\
\hline -Pneumonia \& Influenza & $480-488$ & 149 & 10.8 & 8.8 & 13.4 \\
\hline -Chronic Obstructive Dis & $490-496$ & 92 & 7.7 & 5.9 & 10.2 \\
\hline -Lung Dis from External Agents & $500-508$ & 4 & 0.2 & 0.1 & 0.6 \\
\hline -Other Respiratory Dis & $510-519$ & 27 & 2.0 & 1.4 & 3.0 \\
\hline DIGESTIVE SYSTEM & $520-579$ & 389 & 33.0 & 28.7 & 38.0 \\
\hline -Oral Cavity, Saliva Glands, Jaw & $520-529$ & 61 & 7.4 & 5.1 & 10.6 \\
\hline -Esophagus, Stomach, Duodenum & $530-538$ & 71 & 5.6 & 4.0 & 7.8 \\
\hline -Appendicitis & $540-543$ & 11 & 0.6 & 0.3 & 1.1 \\
\hline -Hernias & $550-553$ & 71 & 5.3 & 4.0 & 7.1 \\
\hline -Enteritis, Colitis & $555-558$ & 25 & 1.9 & 1.1 & 3.2 \\
\hline -Other Intestinal Dis & $560-569$ & 85 & 7.4 & 5.4 & 10.1 \\
\hline -Other Digestive Dis & $570-579$ & 65 & 5.0 & 3.6 & 6.8 \\
\hline GENITOURINARY SYSTEM & $580-629$ & 287 & 25.3 & 21.5 & 29.7 \\
\hline -Nephritis, Nephrosis & \begin{tabular}{|l|}
$580-589$ \\
\end{tabular} & 9 & 0.5 & 0.2 & 1.0 \\
\hline -Other Urinary Dis & $590-599$ & 176 & 14.1 & 11.6 & 17.1 \\
\hline -Male Genital Organ Dis & $600-608$ & 28 & 2.1 & 1.2 & 3.7 \\
\hline
\end{tabular}

(Continued)

*Only those diagnostic categories with at least one occurrence appear in this table.

**Standardized to age distribution of 2000 U.S. population. 


\section{Savannah River Site 2010}

\section{Absence Data}

Appendix K. Age-Adjusted IIIness and Injury Rates by Diagnostic Category*

Part 3. Men and Women

\begin{tabular}{|c|c|c|c|c|c|}
\hline & & $\begin{array}{l}\text { Number of } \\
\text { Diagnoses }\end{array}$ & $\begin{array}{c}\text { Age-Adjusted } \\
\text { Rate per } 1,000 * *\end{array}$ & $\begin{array}{c}\text { Lower } 95 \% \\
\text { Confidence } \\
\text { Limit per 1,000 }\end{array}$ & $\begin{array}{c}\text { Upper } 95 \% \\
\text { Confidence } \\
\text { Limit per 1,000 }\end{array}$ \\
\hline Diagnostic Category & ICD-9-CM Code & \multirow[b]{2}{*}{8} & \multirow[b]{2}{*}{0.3} & \multirow[b]{2}{*}{0.2} & \multirow[b]{2}{*}{0.6} \\
\hline -Breast Disorders & $610-612$ & & & & \\
\hline -Pelvic Inflammatory Dis & 614-616 & 6 & 1.6 & 0.7 & 3.8 \\
\hline -Other Female Disorders & $617-629$ & 60 & 6.6 & 4.6 & 9.5 \\
\hline SKIN AND SUBCUTANEOUS TISSUE & 680-709 & 82 & 6.5 & 4.9 & 8.8 \\
\hline -Infections & $680-686$ & 38 & 3.2 & 2.1 & 5.0 \\
\hline -Other Inflammatory Conditions & $690-698$ & 14 & 1.3 & 0.6 & 2.6 \\
\hline -Other & 700-709 & 30 & 2.0 & 1.3 & 3.2 \\
\hline $\begin{array}{l}\text { MUSCULOSKELETAL \& CONNECTIVE } \\
\text { TISSUE }\end{array}$ & 710-739 & 1055 & 83.3 & 76.7 & 90.3 \\
\hline -Arthropathies & $710-719$ & 303 & 22.9 & 19.7 & 26.6 \\
\hline -Dorsopathies & $720-724$ & 528 & 43.2 & 38.4 & 48.6 \\
\hline -Rheumatism, Excluding Back & 725-729 & 187 & 14.0 & 11.6 & 16.8 \\
\hline -Other Dis \& Acquired Deformities & $730-739$ & 37 & 3.2 & 2.1 & 4.9 \\
\hline CONGENITAL ANOMALIES & $740-759$ & 9 & 0.7 & 0.4 & 1.4 \\
\hline $\begin{array}{l}\text { SYMPTOMS, SIGNS, \& ILL-DEFINED } \\
\text { CONDITIONS }\end{array}$ & 780-799 & 1179 & 104.9 & 96.8 & 113.7 \\
\hline -Symptoms & $780-789$ & 1138 & 101.7 & 93.7 & 110.4 \\
\hline -Non-Specific Abnormal Findings & $790-796$ & 41 & 3.2 & 2.0 & 5.0 \\
\hline INJURY \& POISONING & 800-999 & 455 & 40.4 & 35.6 & 46.0 \\
\hline -Fracture - Skull & \begin{tabular}{|l|}
$800-804$ \\
\end{tabular} & 20 & 1.7 & 0.9 & 3.4 \\
\hline -Fracture - Upper Limb & 810-819 & 9 & 1.4 & 0.6 & 3.3 \\
\hline -Fracture - Lower Limb & $820-829$ & 21 & 1.9 & 1.0 & 3.5 \\
\hline -Dislocation & 830-839 & 43 & 3.4 & 2.3 & 5.1 \\
\hline -Sprains \& Strains - Back & 846-847 & 43 & 3.7 & 2.4 & 5.6 \\
\hline -Sprains \& Strains - Other & $840-845,848$ & 90 & 8.1 & 6.1 & 10.7 \\
\hline -Intracranial Injury & $850-854$ & 8 & 0.9 & 0.3 & 2.2 \\
\hline -Internal Injury - Thorax, Abdomen, Pelvis & $860-869$ & 3 & 0.2 & 0.1 & 0.7 \\
\hline -Open Wound - Head, Neck, Trunk & 870-879 & 27 & 2.0 & 1.3 & 3.2 \\
\hline -Late Effects of Accident & 905-909 & 4 & 0.6 & 0.1 & 2.2 \\
\hline -Superficial Injury & $910-919$ & 13 & 1.8 & 0.9 & 3.7 \\
\hline -Contusion & $920-924$ & 29 & 3.8 & 2.3 & 6.4 \\
\hline -Foreign Body Entering Orifice & 930-939 & 1 & 0.0 & 0.0 & 0.3 \\
\hline -Burns & $940-949$ & 12 & 0.9 & 0.5 & 1.5 \\
\hline -Injury to Nerves \& Spinal Cord & $950-957$ & 3 & 0.2 & 0.0 & 0.6 \\
\hline -Complications \& Unspecified Injuries & 958-959 & 8 & 0.5 & 0.2 & 1.1 \\
\hline -Poisoning - Medicinal/Biological & $960-979$ & 5 & 0.3 & 0.1 & 0.7 \\
\hline
\end{tabular}

(Continued)

*Only those diagnostic categories with at least one occurrence appear in this table.

**Standardized to age distribution of 2000 U.S. population. 


\section{Savannah River Site 2010}

\section{Absence Data}

Appendix K. Age-Adjusted IIIness and Injury Rates by Diagnostic Category*

Part 3. Men and Women

\begin{tabular}{|c|c|c|c|c|c|}
\hline & & $\begin{array}{l}\text { Number of } \\
\text { Diagnoses }\end{array}$ & $\begin{array}{c}\text { Age-Adjusted } \\
\text { Rate per } 1,000 * *\end{array}$ & $\begin{array}{c}\text { Lower } 95 \% \\
\text { Confidence } \\
\text { Limit per 1,000 }\end{array}$ & $\begin{array}{c}\text { Upper 95\% } \\
\text { Confidence } \\
\text { Limit per 1,000 }\end{array}$ \\
\hline Diagnostic Category & ICD-9-CM Code & \multirow[b]{2}{*}{2} & \multirow[b]{2}{*}{0.1} & \multirow[b]{2}{*}{0.0} & \multirow[b]{2}{*}{0.6} \\
\hline -Toxic Effects - Non-medicinal & 980-989 & & & & \\
\hline -Unspecified Effects - External Causes & $990-995$ & 17 & 1.4 & 0.7 & 2.7 \\
\hline -Complications of Surgical/Medical Care & 996-999 & 97 & 7.6 & 5.9 & 9.9 \\
\hline Total & & 6010 & 499.9 & 482.8 & 517.7 \\
\hline
\end{tabular}

*Only those diagnostic categories with at least one occurrence appear in this table.

**Standardized to age distribution of 2000 U.S. population. 
Savannah River Site 2010

OSHA Data

Appendix L. Number of Workers with at Least One OSHA Event by Gender, Age, and Job Category*

\begin{tabular}{|c|c|c|c|c|c|c|c|c|c|c|}
\hline \multirow{3}{*}{ Job Category } & \multicolumn{4}{|c|}{ Women } & \multicolumn{5}{|c|}{ Men } & \multirow[b]{3}{*}{ TOTAL } \\
\hline & \multicolumn{3}{|c|}{ Age Group } & \multirow[b]{2}{*}{ TOTAL } & \multicolumn{4}{|c|}{ Age Group } & \multirow[b]{2}{*}{ TOTAL } & \\
\hline & $30-39$ & $40-49$ & $50+$ & & $16-29$ & $30-39$ & $40-49$ & $50+$ & & \\
\hline Professional & 0 & 0 & 2 & 2 & 0 & 0 & 0 & 0 & 0 & 2 \\
\hline Administrative Support & 1 & 1 & 1 & 3 & 0 & 0 & 0 & 0 & 0 & 3 \\
\hline Technical Support & 0 & 0 & 1 & 1 & 0 & 0 & 0 & 1 & 1 & 2 \\
\hline Security and Fire & 0 & 0 & 0 & 0 & 0 & 1 & 0 & 0 & 1 & 1 \\
\hline Crafts & 2 & 0 & 0 & 2 & 2 & 0 & 4 & 1 & 7 & 9 \\
\hline Line Operators & 0 & 0 & 0 & 0 & 0 & 1 & 1 & 3 & 5 & 5 \\
\hline TOTAL & 3 & 1 & 4 & 8 & 2 & 2 & 5 & 5 & 14 & 22 \\
\hline
\end{tabular}

*Only those job categories and gender/age combinations with at least one OSHA event appear in this table.

Appendix M. Total Number of Workdays Lost or with Restricted Activity from OSHA Events by Gender and Age

\begin{tabular}{|c|c|c|c|c|c|c|c|c|c|}
\hline \multirow[b]{2}{*}{ Age Group } & \multicolumn{3}{|c|}{ Women } & \multicolumn{3}{|c|}{ Men } & \multicolumn{3}{|c|}{ TOTAL } \\
\hline & $\begin{array}{c}\text { Number of } \\
\text { Events }\end{array}$ & \begin{tabular}{|c} 
Days \\
Restricted
\end{tabular} & Days Lost & $\begin{array}{c}\text { Number of } \\
\text { Events }\end{array}$ & $\begin{array}{c}\text { Days } \\
\text { Restricted }\end{array}$ & Days Lost & $\begin{array}{c}\text { Number of } \\
\text { Events }\end{array}$ & $\begin{array}{c}\text { Days } \\
\text { Restricted }\end{array}$ & Days Lost \\
\hline $16-29$ & 0 & 0 & 0 & 2 & 0 & 0 & 2 & 0 & 0 \\
\hline $30-39$ & 3 & 159 & 0 & 2 & 180 & 0 & 5 & 339 & 0 \\
\hline $40-49$ & 1 & 0 & 0 & 6 & 151 & 0 & 7 & 151 & 0 \\
\hline $50+$ & 4 & 28 & 71 & 5 & 105 & 0 & 9 & 133 & 71 \\
\hline TOTAL & 8 & 187 & 71 & 15 & 436 & 0 & 23 & 623 & 71 \\
\hline
\end{tabular}

Appendix N. Total Number of Workdays Lost or with Restricted Activity from OSHA Events by Gender and Job Category*

\begin{tabular}{|c|c|c|c|c|c|c|c|c|c|}
\hline Job Category & \multicolumn{3}{|c|}{ Women } & \multicolumn{3}{|c|}{ Men } & \multicolumn{3}{|c|}{ TOTAL } \\
\hline $\begin{array}{l}\text { Administrative } \\
\text { Support }\end{array}$ & 3 & 28 & 71 & 0 & 0 & 0 & 3 & 28 & 71 \\
\hline Crafts & 2 & 159 & 0 & 8 & 256 & 0 & 10 & 415 & 0 \\
\hline Line Operators & 0 & 0 & 0 & 5 & 180 & 0 & 5 & 180 & 0 \\
\hline TOTAL & 8 & 187 & 71 & 15 & 436 & 0 & 23 & 623 & 71 \\
\hline
\end{tabular}

*Only those job categories with at least one OSHA event appear in this table. 
Savannah River Site 2010

OSHA Data

Appendix O. Number of Diagnoses in Each Diagnostic Category by Gender and Age*

\begin{tabular}{|c|c|c|c|c|c|}
\hline & & \multicolumn{4}{|c|}{ Women } \\
\hline & & \multicolumn{3}{|c|}{ Age Group } & \multirow[b]{2}{*}{ TOTAL } \\
\hline & & $30-39$ & $40-49$ & $50+$ & \\
\hline Diagnostic Category & ICD-9-CM code & \multirow[b]{2}{*}{0} & \multirow[b]{2}{*}{0} & \multirow[b]{2}{*}{0} & \multirow[b]{2}{*}{0} \\
\hline NERVOUS SYSTEM (NS) \& SENSE ORGANS & $320-389$ & & & & \\
\hline -Other Headache Syndromes & 339 & 0 & 0 & 0 & 0 \\
\hline $\begin{array}{l}\text { MUSCULOSKELETAL \& CONNECTIVE } \\
\text { TISSUE }\end{array}$ & 710-739 & 1 & 0 & 0 & 1 \\
\hline -Rheumatism, Excluding Back & 725-729 & 1 & 0 & 0 & 1 \\
\hline $\begin{array}{l}\text { SYMPTOMS, SIGNS, \& ILL-DEFINED } \\
\text { CONDITIONS }\end{array}$ & $780-799$ & 0 & 1 & 0 & 1 \\
\hline -Symptoms & $780-789$ & 0 & 1 & 0 & 1 \\
\hline INJURY \& POISONING & $800-999$ & 5 & 1 & 7 & 13 \\
\hline -Fracture - Upper Limb & \begin{tabular}{|l|}
$810-819$ \\
\end{tabular} & 0 & 0 & 0 & 0 \\
\hline -Dislocation & 830-839 & 1 & 0 & 1 & 2 \\
\hline -Sprains \& Strains - Back & 846-847 & 0 & 0 & 2 & 2 \\
\hline -Sprains \& Strains - Other & $840-845,848$ & 2 & 0 & 1 & 3 \\
\hline -Open Wound - Upper Limb & $880-887$ & 0 & 0 & 0 & 0 \\
\hline -Superficial Injury & $910-919$ & 2 & 0 & 0 & 2 \\
\hline -Contusion & 920-924 & 0 & 1 & 2 & 3 \\
\hline -Foreign Body Entering Orifice & 930-939 & 0 & 0 & 0 & 0 \\
\hline -Burns & $940-949$ & 0 & 0 & 0 & 0 \\
\hline -Toxic Effects - Non-medicinal & $980-989$ & 0 & 0 & 0 & 0 \\
\hline -Unspecified Effects - External Causes & $990-995$ & 0 & 0 & 1 & 1 \\
\hline
\end{tabular}

*Only those diagnostic categories and gender/age combinations with at least one OSHA event appear in this table. 


\section{Savannah River Site 2010}

OSHA Data

Appendix O. Number of Diagnoses in Each Diagnostic Category by Gender and Age*

\begin{tabular}{|c|c|c|c|c|c|c|c|}
\hline & & \multicolumn{5}{|c|}{ Men } & \multirow[b]{3}{*}{ TOTAL } \\
\hline & & \multicolumn{4}{|c|}{ Age Group } & \multirow[b]{2}{*}{ TOTAL } & \\
\hline & & $16-29$ & 30 - 39 & $40-49$ & $50+$ & & \\
\hline Diagnostic Category & ICD-9-CM code & & & & & & \\
\hline NERVOUS SYSTEM (NS) \& SENSE ORGANS & $320-389$ & 0 & 0 & 1 & 0 & 1 & 1 \\
\hline -Other Headache Syndromes & 339 & 0 & 0 & 1 & 0 & 1 & 1 \\
\hline $\begin{array}{l}\text { MUSCULOSKELETAL \& CONNECTIVE } \\
\text { TISSUE }\end{array}$ & 710-739 & 0 & 0 & 0 & 0 & 0 & 1 \\
\hline -Rheumatism, Excluding Back & 725-729 & 0 & 0 & 0 & 0 & 0 & 1 \\
\hline $\begin{array}{l}\text { SYMPTOMS, SIGNS, \& ILL-DEFINED } \\
\text { CONDITIONS }\end{array}$ & $780-799$ & 0 & 0 & 0 & 0 & 0 & 1 \\
\hline -Symptoms & $780-789$ & 0 & 0 & 0 & 0 & 0 & 1 \\
\hline INJURY \& POISONING & $800-999$ & 2 & 2 & 8 & 5 & 17 & 30 \\
\hline -Fracture - Upper Limb & 810-819 & 0 & 0 & 2 & 0 & 2 & 2 \\
\hline -Dislocation & 830-839 & 0 & 0 & 0 & 0 & 0 & 2 \\
\hline -Sprains \& Strains - Back & 846-847 & 0 & 0 & 0 & 2 & 2 & 4 \\
\hline -Sprains \& Strains - Other & $840-845,848$ & 0 & 0 & 1 & 0 & 1 & 4 \\
\hline -Open Wound - Upper Limb & 880-887 & 1 & 1 & 3 & 1 & 6 & 6 \\
\hline -Superficial Injury & $910-919$ & 0 & 0 & 0 & 0 & 0 & 2 \\
\hline -Contusion & 920-924 & 0 & 0 & 1 & 0 & 1 & 4 \\
\hline -Foreign Body Entering Orifice & 930-939 & 1 & 0 & 0 & 0 & 1 & 1 \\
\hline -Burns & $940-949$ & 0 & 0 & 0 & 1 & 1 & 1 \\
\hline -Toxic Effects - Non-medicinal & $980-989$ & 0 & 0 & 1 & 1 & 2 & 2 \\
\hline -Unspecified Effects - External Causes & $990-995$ & 0 & 1 & 0 & 0 & 1 & 2 \\
\hline
\end{tabular}

\begin{tabular}{|c|c|c|c|c|c|c|c|c|c|c|}
\hline & \multicolumn{4}{|c|}{ Women } & \multicolumn{5}{|c|}{ Men } & \multirow[b]{3}{*}{ TOTAL } \\
\hline & \multicolumn{3}{|c|}{ Age Group } & \multirow[b]{2}{*}{ TOTAL } & \multicolumn{4}{|c|}{ Age Group } & \multirow[b]{2}{*}{ TOTAL } & \\
\hline & $30-39$ & $40-49$ & $50+$ & & $16-29$ & 30 - 39 & $40-49$ & $50+$ & & \\
\hline Diagnostic Category & \multirow[b]{2}{*}{6} & \multirow[b]{2}{*}{2} & \multirow[b]{2}{*}{7} & \multirow[b]{2}{*}{15} & \multirow[b]{2}{*}{2} & \multirow[b]{2}{*}{2} & \multirow[b]{2}{*}{9} & \multirow[b]{2}{*}{5} & \multirow[b]{2}{*}{18} & \multirow[b]{2}{*}{33} \\
\hline Total & & & & & & & & & & \\
\hline
\end{tabular}

*Only those diagnostic categories and gender/age combinations with at least one OSHA event appear in this table. 


\section{Savannah River Site 2010}

OSHA Data

Appendix P. Number of Workdays Lost or with Restricted Activity in Each Diagnostic Category by Gender and Age*

\begin{tabular}{|c|c|c|c|c|c|c|c|}
\hline & & \multicolumn{6}{|c|}{ Women } \\
\hline & & \multicolumn{6}{|c|}{ Age Group } \\
\hline & & \multicolumn{2}{|c|}{$30-39$} & \multicolumn{2}{|c|}{$40-49$} & \multicolumn{2}{|c|}{$\mathbf{5 0 +}$} \\
\hline & & $\begin{array}{c}\text { Days } \\
\text { Restricted }\end{array}$ & Days Lost & \begin{tabular}{|c|} 
Days \\
Restricted
\end{tabular} & Days Lost & \begin{tabular}{|c|} 
Days \\
Restricted
\end{tabular} & Days Lost \\
\hline Diagnostic Category & ICD-9-CM Codes & \multirow[b]{2}{*}{159} & \multirow[b]{2}{*}{0} & \multirow[b]{2}{*}{0} & \multirow[b]{2}{*}{0} & \multirow[b]{2}{*}{0} & \multirow[b]{2}{*}{0} \\
\hline -Rheumatism, Excluding Back & 725-729 & & & & & & \\
\hline -Symptoms & 780-789 & 0 & 0 & 0 & 0 & 0 & 0 \\
\hline -Dislocation & 830-839 & 0 & 0 & 0 & 0 & 28 & 71 \\
\hline -Sprains \& Strains - Back & 846-847 & 0 & 0 & 0 & 0 & 28 & 71 \\
\hline -Sprains \& Strains - Other & $840-845,848$ & 159 & 0 & 0 & 0 & 28 & 71 \\
\hline -Superficial Injury & 910-919 & 0 & 0 & 0 & 0 & 0 & 0 \\
\hline -Contusion & $920-924$ & 0 & 0 & 0 & 0 & 28 & 71 \\
\hline $\begin{array}{l}\text {-Unspecified Effects - External } \\
\text { Causes }\end{array}$ & $990-995$ & 0 & 0 & 0 & 0 & 0 & 0 \\
\hline
\end{tabular}

\begin{tabular}{|c|c|c|c|c|c|c|c|c|c|}
\hline & & \multicolumn{8}{|c|}{ Men } \\
\hline & & \multicolumn{8}{|c|}{ Age Group } \\
\hline & & \multicolumn{2}{|c|}{$16-29$} & \multicolumn{2}{|c|}{30 - 39} & \multicolumn{2}{|c|}{$40-49$} & \multicolumn{2}{|c|}{$50+$} \\
\hline & & $\begin{array}{c}\text { Days } \\
\text { Restricted }\end{array}$ & $\begin{array}{l}\text { Days } \\
\text { Lost }\end{array}$ & $\begin{array}{c}\text { Days } \\
\text { Restricted }\end{array}$ & $\begin{array}{l}\text { Days } \\
\text { Lost }\end{array}$ & $\begin{array}{c}\text { Days } \\
\text { Restricted }\end{array}$ & $\begin{array}{l}\text { Days } \\
\text { Lost }\end{array}$ & $\begin{array}{c}\text { Days } \\
\text { Restricted }\end{array}$ & $\begin{array}{l}\text { Days } \\
\text { Lost }\end{array}$ \\
\hline Diagnostic Category & ICD-9-CM Codes & & & & & & & & \\
\hline -Other Headache Syndromes & 339 & 0 & 0 & 0 & 0 & 0 & 0 & 0 & 0 \\
\hline -Fracture - Upper Limb & 810-819 & 0 & 0 & 0 & 0 & 0 & 0 & 0 & 0 \\
\hline -Sprains \& Strains - Back & $846-847$ & 0 & 0 & 0 & 0 & 0 & 0 & 105 & 0 \\
\hline -Sprains \& Strains - Other & $840-845,848$ & 0 & 0 & 0 & 0 & 151 & 0 & 0 & 0 \\
\hline -Open Wound - Upper Limb & 880-887 & 0 & 0 & 180 & 0 & 0 & 0 & 0 & 0 \\
\hline -Contusion & $920-924$ & 0 & 0 & 0 & 0 & 0 & 0 & 0 & 0 \\
\hline -Foreign Body Entering Orifice & 930-939 & 0 & 0 & 0 & 0 & 0 & 0 & 0 & 0 \\
\hline -Burns & $940-949$ & 0 & 0 & 0 & 0 & 0 & 0 & 0 & 0 \\
\hline -Toxic Effects - Non-medicinal & $980-989$ & 0 & 0 & 0 & 0 & 0 & 0 & 0 & 0 \\
\hline $\begin{array}{l}\text {-Unspecified Effects - External } \\
\text { Causes }\end{array}$ & $990-995$ & 0 & 0 & 0 & 0 & 0 & 0 & 0 & 0 \\
\hline
\end{tabular}

*OSHA events with >1 ICD-9-CM code in the same diagnostic category were counted only once. Only those diagnostic categories and gender/age combinations with at least one occurrence appear in this table. 
Savannah River Site 2010

OSHA Data

Appendix Q. Number of Occurrences in Each Accident Category by Gender and Age*

\begin{tabular}{|c|c|c|c|c|c|c|c|c|c|c|c|}
\hline & & \multicolumn{4}{|c|}{ Women } & \multicolumn{5}{|c|}{ Men } & \multirow[b]{3}{*}{ TOTAL } \\
\hline & & \multicolumn{3}{|c|}{ Age Group } & \multirow[b]{2}{*}{ TOTAL } & \multicolumn{4}{|c|}{ Age Group } & \multirow[b]{2}{*}{ TOTAL } & \\
\hline & & 30 - 39 & $40-49$ & $\begin{array}{l}\mathbf{5 0} \\
+\end{array}$ & & $16-29$ & $30-39$ & $40-49$ & $\begin{array}{l}50 \\
+\end{array}$ & & \\
\hline Type of Accident & E Codes & & & & & & & & & & \\
\hline Accidental Poisoning - Non-medicinal & E860-E869 & 0 & 0 & 0 & 0 & 0 & 0 & 1 & 0 & 1 & 1 \\
\hline Falls & E880-E888 & 0 & 0 & 2 & 2 & 0 & 0 & 0 & 0 & 0 & 2 \\
\hline Natural/Environmental Factors & E900-E909 & 1 & 0 & 0 & 1 & 0 & 1 & 0 & 1 & 2 & 3 \\
\hline $\begin{array}{l}\text { Submersion/Suffocation/Foreign } \\
\text { Bodies }\end{array}$ & E910-E915 & 0 & 0 & 0 & 0 & 1 & 0 & 0 & 0 & 1 & 1 \\
\hline Other Accidents & E916-E928 & 2 & 1 & 1 & 4 & 1 & 1 & 5 & 4 & 11 & 15 \\
\hline
\end{tabular}

*Only those accident types and gender/age combinations with at least one occurrence appear in this table. 
Savannah River Site 2010

OSHA Data

Appendix R. Number of Workdays Lost or with Restricted Activity in Each Accident Category by Gender and Age*

\begin{tabular}{|c|c|c|c|c|c|c|c|}
\hline & & \multicolumn{6}{|c|}{ Women } \\
\hline & & \multicolumn{6}{|c|}{ Age Group } \\
\hline & & \multicolumn{2}{|c|}{30 - 39} & \multicolumn{2}{|c|}{$40-49$} & \multicolumn{2}{|c|}{$50+$} \\
\hline & & $\begin{array}{c}\text { Days } \\
\text { Restricted }\end{array}$ & Days Lost & $\begin{array}{c}\text { Days } \\
\text { Restricted }\end{array}$ & Days Lost & $\begin{array}{c}\text { Days } \\
\text { Restricted }\end{array}$ & Days Lost \\
\hline Type of Accident & E Codes & \multirow[b]{2}{*}{0} & \multirow[b]{2}{*}{0} & \multirow[b]{2}{*}{0} & \multirow[b]{2}{*}{0} & \multirow[b]{2}{*}{28} & \multirow[b]{2}{*}{71} \\
\hline Falls & E880-E888 & & & & & & \\
\hline Natural/Environmental Factors & E900-E909 & 0 & 0 & 0 & 0 & 0 & 0 \\
\hline Other Accidents & E916-E928 & 159 & 0 & 0 & 0 & 0 & 0 \\
\hline
\end{tabular}

\begin{tabular}{|c|c|c|c|c|c|c|c|c|c|}
\hline & & \multicolumn{8}{|c|}{ Men } \\
\hline & & \multicolumn{8}{|c|}{ Age Group } \\
\hline & & \multicolumn{2}{|c|}{$16-29$} & \multicolumn{2}{|c|}{$30-39$} & \multicolumn{2}{|c|}{$40-49$} & \multicolumn{2}{|c|}{$50+$} \\
\hline & & $\begin{array}{c}\text { Days } \\
\text { Restricted }\end{array}$ & $\begin{array}{l}\text { Days } \\
\text { Lost }\end{array}$ & $\begin{array}{c}\text { Days } \\
\text { Restricted }\end{array}$ & $\begin{array}{l}\text { Days } \\
\text { Lost }\end{array}$ & $\begin{array}{c}\text { Days } \\
\text { Restricted }\end{array}$ & $\begin{array}{l}\text { Days } \\
\text { Lost }\end{array}$ & $\begin{array}{c}\text { Days } \\
\text { Restricted }\end{array}$ & $\begin{array}{l}\text { Days } \\
\text { Lost }\end{array}$ \\
\hline Type of Accident & E Codes & & & & & & & & \\
\hline Accidental Poisoning - Non-medicinal & E860-E869 & 0 & 0 & 0 & 0 & 0 & 0 & 0 & 0 \\
\hline Natural/Environmental Factors & E900-E909 & 0 & 0 & 0 & 0 & 0 & 0 & 0 & 0 \\
\hline $\begin{array}{l}\text { Submersion/Suffocation/Foreign } \\
\text { Bodies }\end{array}$ & E910-E915 & 0 & 0 & 0 & 0 & 0 & 0 & 0 & 0 \\
\hline Other Accidents & E916-E928 & 0 & 0 & 180 & 0 & 151 & 0 & 105 & 0 \\
\hline
\end{tabular}

*OSHA events with $>1$ E code in the same accident type were counted only once. Only those accident types and gender/age combinations with at least one occurrence appear in this table. 


\section{Savannah River Site 2010}

\section{OSHA Data}

Appendix S. Number of Diagnoses in Each Diagnostic Category by Gender and Job Category*

\begin{tabular}{|c|c|c|c|c|c|c|}
\hline & & \multicolumn{5}{|c|}{ Women } \\
\hline & & \multicolumn{4}{|c|}{ Job Category } & \multirow[b]{2}{*}{ TOTAL } \\
\hline & & Professional & $\begin{array}{c}\text { Administrative } \\
\text { Support }\end{array}$ & Technical Support & Crafts & \\
\hline Diagnostic Category & ICD-9-CM Code & & & & & \\
\hline $\begin{array}{l}\text { MUSCULOSKELETAL } \\
\text { \& CONNECTIVE } \\
\text { TISSUE }\end{array}$ & $710-739$ & 0 & 0 & 0 & 1 & 1 \\
\hline $\begin{array}{l}\text {-Rheumatism, Excluding } \\
\text { Back }\end{array}$ & $725-729$ & 0 & 0 & 0 & 1 & 1 \\
\hline $\begin{array}{l}\text { SYMPTOMS, } \\
\text { SIGNS, \& ILL-DEFINED } \\
\text { CONDITIONS }\end{array}$ & $780-799$ & 0 & 1 & 0 & 0 & 1 \\
\hline -Symptoms & $780-789$ & 0 & 1 & 0 & 0 & 1 \\
\hline INJURY \& POISONING & $800-999$ & 2 & 7 & 1 & 3 & 13 \\
\hline -Dislocation & $830-839$ & 0 & 2 & 0 & 0 & 2 \\
\hline -Sprains \& Strains - Back & 846-847 & 1 & 1 & 0 & 0 & 2 \\
\hline $\begin{array}{l}\text {-Sprains \& Strains - } \\
\text { Other }\end{array}$ & $840-845,848$ & 0 & 2 & 0 & 1 & 3 \\
\hline -Superficial Injury & 910-919 & 0 & 0 & 0 & 2 & 2 \\
\hline -Contusion & $920-924$ & 1 & 2 & 0 & 0 & 3 \\
\hline $\begin{array}{l}\text {-Unspecified } \\
\text { Effects - External Causes }\end{array}$ & $990-995$ & 0 & 0 & 1 & 0 & 1 \\
\hline
\end{tabular}

\begin{tabular}{|c|c|c|c|c|c|}
\hline & \multicolumn{5}{|c|}{ Women } \\
\hline & \multicolumn{4}{|c|}{ Job Category } & \multirow[b]{2}{*}{ TOTAL } \\
\hline & Professional & $\begin{array}{c}\text { Administrative } \\
\text { Support }\end{array}$ & Technical Support & Crafts & \\
\hline Diagnostic Category & \multirow[b]{2}{*}{2} & \multirow[b]{2}{*}{8} & \multirow[b]{2}{*}{1} & \multirow[b]{2}{*}{4} & \multirow[b]{2}{*}{15} \\
\hline Total & & & & & \\
\hline
\end{tabular}

\begin{tabular}{|c|c|c|c|c|c|c|}
\hline & & \multicolumn{5}{|c|}{ Men } \\
\hline & & \multicolumn{4}{|c|}{ Job Category } & \multirow[b]{2}{*}{ TOTAL } \\
\hline & & Technical Support & \begin{tabular}{|l|} 
Security and Fire \\
\end{tabular} & Crafts & Line Operators & \\
\hline Diagnostic Category & ICD-9-CM Code & & & & & \\
\hline $\begin{array}{l}\text { NERVOUS SYSTEM } \\
\text { (NS) \& SENSE ORGANS }\end{array}$ & 320-389 & 0 & 0 & 1 & 0 & 1 \\
\hline $\begin{array}{l}\text {-Other Headache } \\
\text { Syndromes }\end{array}$ & 339 & 0 & 0 & 1 & 0 & 1 \\
\hline INJURY \& POISONING & $800-999$ & 1 & 1 & 10 & 5 & 17 \\
\hline -Fracture - Upper Limb & 810-819 & 0 & 0 & 2 & 0 & 2 \\
\hline -Sprains \& Strains - Back & 846-847 & 0 & 0 & 1 & 1 & 2 \\
\hline $\begin{array}{l}\text {-Sprains \& Strains - } \\
\text { Other }\end{array}$ & $840-845,848$ & 0 & 0 & 1 & 0 & 1 \\
\hline $\begin{array}{l}\text {-Open Wound - Upper } \\
\text { Limb }\end{array}$ & 880-887 & 0 & 0 & 3 & 3 & 6 \\
\hline
\end{tabular}

(Continued)

*Only those diagnostic categories and gender/job category combinations with at least one occurrence appear in this table. 
Savannah River Site 2010

OSHA Data

Appendix S. Number of Diagnoses in Each Diagnostic Category by Gender and Job Category*

\begin{tabular}{|c|c|c|c|c|c|c|}
\hline & & \multicolumn{5}{|c|}{ Men } \\
\hline & & \multicolumn{4}{|c|}{ Job Category } & \multirow[b]{2}{*}{ TOTAL } \\
\hline & & Technical Support & Security and Fire & Crafts & Line Operators & \\
\hline Diagnostic Category & ICD-9-CM Code & \multirow[b]{2}{*}{0} & \multirow[b]{2}{*}{0} & \multirow[b]{2}{*}{1} & \multirow[b]{2}{*}{0} & \multirow[b]{2}{*}{1} \\
\hline -Contusion & $920-924$ & & & & & \\
\hline $\begin{array}{l}\text {-Foreign Body Entering } \\
\text { Orifice }\end{array}$ & $930-939$ & 0 & 0 & 1 & 0 & 1 \\
\hline -Burns & 940-949 & 1 & 0 & 0 & 0 & 1 \\
\hline $\begin{array}{l}\text {-Toxic } \\
\text { Effects - Non-medicinal }\end{array}$ & $980-989$ & 0 & 0 & 1 & 1 & 2 \\
\hline $\begin{array}{l}\text {-Unspecified } \\
\text { Effects - External Causes }\end{array}$ & $990-995$ & 0 & 1 & 0 & 0 & 1 \\
\hline
\end{tabular}

\begin{tabular}{|c|c|c|c|c|c|}
\hline & \multicolumn{5}{|c|}{ Men } \\
\hline & \multicolumn{4}{|c|}{ Job Category } & \multirow[b]{2}{*}{ TOTAL } \\
\hline & Technical Support & Security and Fire & Crafts & Line Operators & \\
\hline Diagnostic Category & \multirow[b]{2}{*}{1} & \multirow[b]{2}{*}{1} & \multirow[b]{2}{*}{11} & \multirow[b]{2}{*}{5} & \multirow[b]{2}{*}{18} \\
\hline Total & & & & & \\
\hline
\end{tabular}

*Only those diagnostic categories and gender/job category combinations with at least one occurrence appear in this table. 


\section{Savannah River Site 2010}

OSHA Data

Appendix T. Number of Workdays Lost or with Restricted Activity in Each Diagnostic Category by Gender and Job Category*

\begin{tabular}{|c|c|c|c|c|c|c|c|c|c|}
\hline & & \multicolumn{8}{|c|}{ Women } \\
\hline & & \multicolumn{8}{|c|}{ Job Category } \\
\hline & & \multicolumn{2}{|c|}{ Professional } & \multicolumn{2}{|c|}{$\begin{array}{c}\text { Administrative } \\
\text { Support }\end{array}$} & \multicolumn{2}{|c|}{$\begin{array}{l}\text { Technical } \\
\text { Support }\end{array}$} & \multicolumn{2}{|c|}{ Crafts } \\
\hline & & $\begin{array}{c}\text { Days } \\
\text { Restricted }\end{array}$ & $\begin{array}{l}\text { Days } \\
\text { Lost }\end{array}$ & $\begin{array}{c}\text { Days } \\
\text { Restricted }\end{array}$ & $\begin{array}{l}\text { Days } \\
\text { Lost }\end{array}$ & $\begin{array}{c}\text { Days } \\
\text { Restricted }\end{array}$ & $\begin{array}{l}\text { Days } \\
\text { Lost }\end{array}$ & $\begin{array}{c}\text { Days } \\
\text { Restricted }\end{array}$ & $\begin{array}{l}\text { Days } \\
\text { Lost }\end{array}$ \\
\hline Diagnostic Category & ICD-9-CM Codes & & & & & & & & \\
\hline -Rheumatism, Excluding Back & 725-729 & 0 & 0 & 0 & 0 & 0 & 0 & 159 & 0 \\
\hline -Symptoms & $780-789$ & 0 & 0 & 0 & 0 & 0 & 0 & 0 & 0 \\
\hline -Dislocation & $830-839$ & 0 & 0 & 28 & 71 & 0 & 0 & 0 & 0 \\
\hline -Sprains \& Strains - Back & $846-847$ & 0 & 0 & 28 & 71 & 0 & 0 & 0 & 0 \\
\hline -Sprains \& Strains - Other & $840-845,848$ & 0 & 0 & 28 & 71 & 0 & 0 & 159 & 0 \\
\hline -Superficial Injury & $910-919$ & 0 & 0 & 0 & 0 & 0 & 0 & 0 & 0 \\
\hline -Contusion & $920-924$ & 0 & 0 & 28 & 71 & 0 & 0 & 0 & 0 \\
\hline $\begin{array}{l}\text {-Unspecified Effects - External } \\
\text { Causes }\end{array}$ & $990-995$ & 0 & 0 & 0 & 0 & 0 & 0 & 0 & 0 \\
\hline
\end{tabular}

\begin{tabular}{|c|c|c|c|c|c|c|c|c|c|}
\hline & & \multicolumn{8}{|c|}{ Men } \\
\hline & & \multicolumn{8}{|c|}{ Job Category } \\
\hline & & \multicolumn{2}{|c|}{ Technical Support } & \multicolumn{2}{|c|}{ Security and Fire } & \multicolumn{2}{|c|}{ Crafts } & \multicolumn{2}{|c|}{ Line Operators } \\
\hline & & $\begin{array}{c}\text { Days } \\
\text { Restricted }\end{array}$ & $\begin{array}{l}\text { Days } \\
\text { Lost }\end{array}$ & $\begin{array}{c}\text { Days } \\
\text { Restricted }\end{array}$ & $\begin{array}{l}\text { Days } \\
\text { Lost }\end{array}$ & \begin{tabular}{c|} 
Days \\
Restricted
\end{tabular} & $\begin{array}{l}\text { Days } \\
\text { Lost }\end{array}$ & \begin{tabular}{c|} 
Days \\
Restricted
\end{tabular} & $\begin{array}{l}\text { Days } \\
\text { Lost }\end{array}$ \\
\hline Diagnostic Category & ICD-9-CM Codes & \multirow[b]{2}{*}{0} & \multirow[b]{2}{*}{0} & \multirow[b]{2}{*}{0} & \multirow[b]{2}{*}{0} & \multirow[b]{2}{*}{0} & \multirow[b]{2}{*}{0} & \multirow[b]{2}{*}{0} & \multirow[b]{2}{*}{0} \\
\hline -Other Headache Syndromes & 339 & & & & & & & & \\
\hline -Fracture - Upper Limb & 810-819 & 0 & 0 & 0 & 0 & 0 & 0 & 0 & 0 \\
\hline -Sprains \& Strains - Back & $846-847$ & 0 & 0 & 0 & 0 & 105 & 0 & 0 & 0 \\
\hline -Sprains \& Strains - Other & $840-845,848$ & 0 & 0 & 0 & 0 & 151 & 0 & 0 & 0 \\
\hline -Open Wound - Upper Limb & 880-887 & 0 & 0 & 0 & 0 & 0 & 0 & 180 & 0 \\
\hline -Contusion & $920-924$ & 0 & 0 & 0 & 0 & 0 & 0 & 0 & 0 \\
\hline -Foreign Body Entering Orifice & 930-939 & 0 & 0 & 0 & 0 & 0 & 0 & 0 & 0 \\
\hline -Burns & $940-949$ & 0 & 0 & 0 & 0 & 0 & 0 & 0 & 0 \\
\hline -Toxic Effects - Non-medicinal & $980-989$ & 0 & 0 & 0 & 0 & 0 & 0 & 0 & 0 \\
\hline $\begin{array}{l}\text {-Unspecified Effects - External } \\
\text { Causes }\end{array}$ & $990-995$ & 0 & 0 & 0 & 0 & 0 & 0 & 0 & 0 \\
\hline
\end{tabular}

*OSHA events with >1 ICD-9-CM code in the same diagnostic category were counted only once. Only those diagnostic categories and gender/job category combinations with at least one occurrence appear in this table. 
Savannah River Site 2010

OSHA Data

Appendix U. Number of Occurrences in Each Accident Category by Gender and Job Category*

\begin{tabular}{|c|c|c|c|c|c|}
\hline & & \multicolumn{4}{|c|}{ Women } \\
\hline & & \multicolumn{3}{|c|}{ Job Category } & \multirow[b]{2}{*}{ TOTAL } \\
\hline & & Professional & $\begin{array}{c}\text { Administrative } \\
\text { Support }\end{array}$ & Crafts & \\
\hline Type of Accident & E CODES & \multirow[b]{2}{*}{1} & \multirow[b]{2}{*}{1} & \multirow[b]{2}{*}{0} & \multirow[b]{2}{*}{2} \\
\hline Falls & E880-E888 & & & & \\
\hline Natural/Environmental Factors & E900-E909 & 0 & 0 & 1 & 1 \\
\hline Other Accidents & E916-E928 & 1 & 2 & 1 & 4 \\
\hline
\end{tabular}

\begin{tabular}{|c|c|c|c|c|c|c|}
\hline & & \multicolumn{5}{|c|}{ Men } \\
\hline & & \multicolumn{4}{|c|}{ Job Category } & \multirow[b]{2}{*}{ TOTAL } \\
\hline & & $\begin{array}{l}\text { Technical } \\
\text { Support }\end{array}$ & $\begin{array}{c}\text { Security and } \\
\text { Fire }\end{array}$ & Crafts & $\begin{array}{c}\text { Line } \\
\text { Operators }\end{array}$ & \\
\hline Type of Accident & E CODES & \multirow[b]{2}{*}{0} & \multirow[b]{2}{*}{0} & \multirow[b]{2}{*}{1} & \multirow[b]{2}{*}{0} & \multirow[b]{2}{*}{1} \\
\hline Accidental Poisoning - Non-medicinal & E860-E869 & & & & & \\
\hline Natural/Environmental Factors & E900-E909 & 0 & 1 & 0 & 1 & 2 \\
\hline $\begin{array}{l}\text { Submersion/Suffocation/Foreign } \\
\text { Bodies }\end{array}$ & E910-E915 & 0 & 0 & 1 & 0 & 1 \\
\hline Other Accidents & E916-E928 & 1 & 0 & 6 & 4 & 11 \\
\hline
\end{tabular}

*Only those accident types and gender/job category combinations with at least one occurrence appear in this table. 


\section{Savannah River Site 2010}

OSHA Data

Appendix V. Number of Workdays Lost or with Restricted Activity in Each Accident Category by Gender and Job Category*

\begin{tabular}{|c|c|c|c|c|c|c|c|}
\hline & & \multicolumn{6}{|c|}{ Women } \\
\hline & & \multicolumn{6}{|c|}{ Job Category } \\
\hline & & \multicolumn{2}{|c|}{ Professional } & \multicolumn{2}{|c|}{$\begin{array}{c}\text { Administrative } \\
\text { Support }\end{array}$} & \multicolumn{2}{|c|}{ Crafts } \\
\hline & & $\begin{array}{c}\text { Days } \\
\text { Restricted }\end{array}$ & Days Lost & $\begin{array}{c}\text { Days } \\
\text { Restricted }\end{array}$ & Days Lost & $\begin{array}{c}\text { Days } \\
\text { Restricted }\end{array}$ & Days Lost \\
\hline Type of Accident & E Codes & \multirow[b]{2}{*}{0} & \multirow[b]{2}{*}{0} & \multirow[b]{2}{*}{28} & \multirow[b]{2}{*}{71} & \multirow[b]{2}{*}{0} & \multirow[b]{2}{*}{0} \\
\hline Falls & E880-E888 & & & & & & \\
\hline Natural/Environmental Factors & E900-E909 & 0 & 0 & 0 & 0 & 0 & 0 \\
\hline Other Accidents & E916-E928 & 0 & 0 & 0 & 0 & 159 & 0 \\
\hline
\end{tabular}

\begin{tabular}{|c|c|c|c|c|c|c|c|c|c|}
\hline & & \multicolumn{8}{|c|}{ Men } \\
\hline & & \multicolumn{8}{|c|}{ Job Category } \\
\hline & & \multicolumn{2}{|c|}{ Technical Support } & \multicolumn{2}{|c|}{ Security and Fire } & \multicolumn{2}{|c|}{ Crafts } & \multicolumn{2}{|c|}{ Line Operators } \\
\hline & & $\begin{array}{c}\text { Days } \\
\text { Restricted }\end{array}$ & $\begin{array}{l}\text { Days } \\
\text { Lost }\end{array}$ & $\begin{array}{c}\text { Days } \\
\text { Restricted }\end{array}$ & $\begin{array}{l}\text { Days } \\
\text { Lost }\end{array}$ & $\begin{array}{c}\text { Days } \\
\text { Restricted }\end{array}$ & $\begin{array}{l}\text { Days } \\
\text { Lost }\end{array}$ & $\begin{array}{c}\text { Days } \\
\text { Restricted }\end{array}$ & $\begin{array}{l}\text { Days } \\
\text { Lost }\end{array}$ \\
\hline Type of Accident & E Codes & \multirow[b]{2}{*}{0} & \multirow[b]{2}{*}{0} & \multirow[b]{2}{*}{0} & \multirow[b]{2}{*}{0} & \multirow[b]{2}{*}{0} & \multirow[b]{2}{*}{0} & \multirow[b]{2}{*}{0} & \multirow[b]{2}{*}{0} \\
\hline Accidental Poisoning - Non-medicinal & E860-E869 & & & & & & & & \\
\hline Natural/Environmental Factors & E900-E909 & 0 & 0 & 0 & 0 & 0 & 0 & 0 & 0 \\
\hline $\begin{array}{l}\text { Submersion/Suffocation/Foreign } \\
\text { Bodies }\end{array}$ & E910-E915 & 0 & 0 & 0 & 0 & 0 & 0 & 0 & 0 \\
\hline Other Accidents & E916-E928 & 0 & 0 & 0 & 0 & 256 & 0 & 180 & 0 \\
\hline
\end{tabular}

*OSHA events with >1 E code in the same accident type were counted only once. Only those accident types and gender/job category combinations with at least one occurrence appear in this table. 
Savannah River Site 2010

OSHA Data

Appendix W. Age-Adjusted OSHA Illness and Injury Rates by Diagnostic Category*

Part 1. Men

\begin{tabular}{|c|c|c|c|c|c|}
\hline & & $\begin{array}{c}\text { Number of } \\
\text { Diagnoses }\end{array}$ & $\begin{array}{c}\text { Age-Adjusted } \\
\text { Rate per } 1,000 * *\end{array}$ & $\begin{array}{c}\text { Lower } 95 \% \\
\text { Confidence } \\
\text { Limit per 1,000 }\end{array}$ & $\begin{array}{c}\text { Upper } 95 \% \\
\text { Confidence } \\
\text { Limit per } 1,000\end{array}$ \\
\hline Diagnostic Category & ICD-9-CM Code & \multirow[b]{2}{*}{1} & \multirow[b]{2}{*}{0.1} & \multirow[b]{2}{*}{0.0} & \multirow[b]{2}{*}{0.8} \\
\hline NERVOUS SYSTEM (NS) \& SENSE ORGANS & $320-389$ & & & & \\
\hline -Other Headache Syndromes & 339 & 1 & 0.1 & 0.0 & 0.8 \\
\hline INJURY \& POISONING & 800-999 & 17 & 3.0 & 1.6 & 5.8 \\
\hline -Fracture - Upper Limb & $810-819$ & 2 & 0.2 & 0.1 & 0.9 \\
\hline -Sprains \& Strains - Back & 846-847 & 2 & 0.1 & 0.0 & 0.4 \\
\hline -Sprains \& Strains - Other & $840-845,848$ & 1 & 0.1 & 0.0 & 0.8 \\
\hline -Open Wound - Upper Limb & $880-887$ & 6 & 1.3 & 0.5 & 3.7 \\
\hline -Contusion & $920-924$ & 1 & 0.1 & 0.0 & 0.8 \\
\hline -Foreign Body Entering Orifice & 930-939 & 1 & 0.5 & 0.1 & 3.6 \\
\hline -Burns & $940-949$ & 1 & 0.0 & 0.0 & 0.3 \\
\hline -Toxic Effects - Non-medicinal & $980-989$ & 3 & 0.6 & 0.1 & 2.6 \\
\hline Total & & 18 & 3.2 & 1.7 & 5.9 \\
\hline
\end{tabular}

* Only those diagnostic categories with at least one occurrence appear in this table.

**Standardized to age distribution of 2000 U.S. population. 
Savannah River Site 2010

OSHA Data

Appendix W. Age-Adjusted OSHA Illness and Injury Rates by Diagnostic Category*

Part 2. Women

\begin{tabular}{|c|c|c|c|c|c|}
\hline & & $\begin{array}{l}\text { Number of } \\
\text { Diagnoses }\end{array}$ & $\begin{array}{c}\text { Age-Adjusted } \\
\text { Rate per } 1,000 * *\end{array}$ & $\begin{array}{c}\text { Lower } 95 \% \\
\text { Confidence } \\
\text { Limit per 1,000 }\end{array}$ & $\begin{array}{c}\text { Upper 95\% } \\
\text { Confidence } \\
\text { Limit per 1,000 }\end{array}$ \\
\hline Diagnostic Category & ICD-9-CM Code & & & & \\
\hline $\begin{array}{l}\text { MUSCULOSKELETAL \& CONNECTIVE } \\
\text { TISSUE }\end{array}$ & $710-739$ & 1 & 1.3 & 0.2 & 9.4 \\
\hline -Rheumatism, Excluding Back & $725-729$ & 1 & 1.3 & 0.2 & 9.4 \\
\hline $\begin{array}{l}\text { SYMPTOMS, SIGNS, \& ILL-DEFINED } \\
\text { CONDITIONS }\end{array}$ & $780-799$ & 1 & 0.3 & 0.0 & 2.4 \\
\hline -Symptoms & 780-789 & 1 & 0.3 & 0.0 & 2.4 \\
\hline INJURY \& POISONING & $800-999$ & 13 & 10.5 & 5.7 & 19.6 \\
\hline -Dislocation & 830-839 & 2 & 2.0 & 0.5 & 8.6 \\
\hline -Sprains \& Strains - Back & 846-847 & 2 & 0.8 & 0.2 & 4.0 \\
\hline -Sprains \& Strains - Other & $840-845,848$ & 3 & 3.3 & 1.0 & 10.7 \\
\hline -Superficial Injury & $910-919$ & 2 & 2.7 & 0.7 & 10.6 \\
\hline -Contusion & $920-924$ & 3 & 1.6 & 0.5 & 5.2 \\
\hline -Toxic Effects - Non-medicinal & \begin{tabular}{|c|}
$980-989$ \\
\end{tabular} & 1 & 0.2 & 0.0 & 1.2 \\
\hline Total & & 15 & 12.2 & 6.8 & 21.8 \\
\hline
\end{tabular}

* Only those diagnostic categories with at least one occurrence appear in this table.

**Standardized to age distribution of 2000 U.S. population. 
Savannah River Site 2010

OSHA Data

Appendix W. Age-Adjusted OSHA Illness and Injury Rates by Diagnostic Category*

Part 3. Men and Women

\begin{tabular}{|c|c|c|c|c|c|}
\hline & & $\begin{array}{l}\text { Number of } \\
\text { Diagnoses }\end{array}$ & $\begin{array}{c}\text { Age-Adjusted } \\
\text { Rate per } 1,000 * *\end{array}$ & $\begin{array}{c}\text { Lower } 95 \% \\
\text { Confidence } \\
\text { Limit per 1,000 }\end{array}$ & $\begin{array}{c}\text { Upper } 95 \% \\
\text { Confidence } \\
\text { Limit per 1,000 }\end{array}$ \\
\hline Diagnostic Category & ICD-9-CM Code & \multirow[b]{2}{*}{1} & \multirow[b]{2}{*}{0.1} & \multirow[b]{2}{*}{0.0} & \multirow[b]{2}{*}{0.6} \\
\hline NERVOUS SYSTEM (NS) \& SENSE ORGANS & 320-389 & & & & \\
\hline -Other Headache Syndromes & 339 & 1 & 0.1 & 0.0 & 0.6 \\
\hline $\begin{array}{l}\text { MUSCULOSKELETAL \& CONNECTIVE } \\
\text { TISSUE }\end{array}$ & 710-739 & 1 & 0.3 & 0.0 & 2.3 \\
\hline -Rheumatism, Excluding Back & 725-729 & 1 & 0.3 & 0.0 & 2.3 \\
\hline $\begin{array}{l}\text { SYMPTOMS, SIGNS, \& ILL-DEFINED } \\
\text { CONDITIONS }\end{array}$ & $780-799$ & 1 & 0.1 & 0.0 & 0.6 \\
\hline -Symptoms & 780-789 & 1 & 0.1 & 0.0 & 0.6 \\
\hline INJURY \& POISONING & $800-999$ & 30 & 4.5 & 2.9 & 7.2 \\
\hline -Fracture - Upper Limb & $810-819$ & 2 & 0.2 & 0.0 & 0.7 \\
\hline -Dislocation & 830-839 & 2 & 0.4 & 0.1 & 2.0 \\
\hline -Sprains \& Strains - Back & 846-847 & 4 & 0.2 & 0.1 & 0.6 \\
\hline -Sprains \& Strains - Other & $840-845,848$ & 4 & 0.8 & 0.3 & 2.5 \\
\hline -Open Wound - Upper Limb & 880-887 & 6 & 1.0 & 0.4 & 2.8 \\
\hline -Superficial Injury & $910-919$ & 2 & 0.6 & 0.2 & 2.5 \\
\hline -Contusion & $920-924$ & 4 & 0.4 & 0.1 & 1.0 \\
\hline -Foreign Body Entering Orifice & 930-939 & 1 & 0.4 & 0.1 & 2.7 \\
\hline -Burns & 940-949 & 1 & 0.0 & 0.0 & 0.3 \\
\hline -Toxic Effects - Non-medicinal & $980-989$ & 4 & 0.5 & 0.1 & 1.9 \\
\hline Total & & 33 & 5.0 & 3.3 & 7.7 \\
\hline
\end{tabular}

* Only those diagnostic categories with at least one occurrence appear in this table.

**Standardized to age distribution of 2000 U.S. population. 